\title{
Micromanaging Freeze Tolerance: The Biogenesis and Regulation of microRNAs in Frozen Frogs
}

\author{
By \\ Hanane Hadj-Moussa \\ B. Sc. Honours, Carleton University, 2015
}

A Thesis Submitted to the Faculty of Graduate Studies and Research in Partial Fulfillment of the Requirements for the Degree of

\author{
Master of Science \\ Department of Biology
}

Carleton University

Ottawa, Ontario, Canada

C) Copyright 2017

Hanane Hadj-Moussa 
The undersigned hereby recommend to the Faculty of Graduate Studies and Research acceptance of this thesis

\title{
Micromanaging Freeze Tolerance: The Biogenesis and Regulation of microRNAs in Frozen Frogs
}

\author{
Submitted by \\ Hanane Hadj-Moussa, B.Sc. \\ in partial fulfillment of the requirements for the degree of Master of Science
}

Chair, Department of Biology

Thesis Supervisor

Carleton University 


\section{ABSTRACT}

When temperatures plummet below $0^{\circ} \mathrm{C}$, the wood frog (Rana sylvatica) freezes up to $65 \%$ of its body water in extracellular ice masses, displaying no measurable brain activity, no breathing, and a flat-lined heart. Various molecular mechanisms including microRNAs, a multifunctional group of short non-coding RNAs, are in place to facilitate freeze tolerance. This thesis provides the first large-scale investigation of microRNA in a freeze tolerant vertebrate. Immunoblotting was used to investigate protein abundance of key microRNA biogenesis factors in brain and liver of control, $24 \mathrm{~h}$ frozen, and $8 \mathrm{~h}$ thawed $R$. sylvatica. Biogenesis capacity was reduced in brains and elevated in livers during freezing and thawing. This correlated with RT-qPCR levels of $\sim 110$ microRNAs, where the majority of differentially expressed miRNAs were downregulated in brains and upregulated in livers. Bioinformatic miRNA targeting predicted brain miRNAs to play a neuroprotective role, while hepatic miRNAs suppressed energy-expensive pro-growth processes. 


\section{PREFACE}

This integrated Master's thesis is composed of two main research papers that are currently awaiting submission to the following journals:

Biochimica et Biophysica Acta - Gene Regulatory Networks

Hadj-Moussa, H. and Storey, K.B. (2017). Micromanaging freeze tolerance: The biogenesis and regulation of neuroprotective microRNAs in frozen brains.

Journal of Molecular and Cell Biology

Hadj-Moussa, H. and Storey, K.B. (2017). Biogenesis and regulation of the freeze-thaw responsive microRNA fingerprint in wood frog liver. 


\section{ACKNOWLEDGEMENTS}

I would like to seriously thank Dr. Ken Storey for being a spectacular, albeit slightly unorthodox, supervisor. Ken, thanks for all the science, the "teachable moments", and the relentless thwarting that have made me into the scientist I am now. Only time will tell what kind of scientist that is... I now appreciate how you have molded me into the "idiot savant, but with 99\% idiot" that I am now, more toys could have probably helped with that. Equal thanks go to Jan Storey for everything! Jan, thanks for answering my relentless barrage of questions, for keeping me inline with pancakes, and for teaching me how to make reviewer responses PG-13. I look forward to annoying you both into an early retirement over the next couple of years.

Now I must thank all the Storey lab members that made my time in the lab a blast... Except for Sam W., Alex W., and Toby... No guys. Just, No. To everyone else, thanks for all the advice, scientific (and not so scientific) discussions, and excursions. Thanks to Ryan G. and Kama for being great friends. Rasha don't worry, my thesis is gluten-free so you should have no issues reading it. I grudgingly also thank Sanoji for forcing and annoying me into writing my thesis, and I thought Ken and Jan were bad... Thanks to Liam and Sam for our early morning chats and for squids and robots! A big thanks to everyone for the scavenged lunches and free rides! A few of the names you've given me over the years that I've learned to stop resisting include; Hananigans, Haynin, Hans, Alejanandro, HaNon, The Official Opposition, and HaJing. And with that, I should also thank Jing- Thanks. And to Saumya (Level 3 acquaintance) for all the late-night chats and constant entertainment.

Special thanks to the high-salt bat cave; To Bryan for teaching me what I know, to Christie and her random videos, to Stuart and his Spitz (but more importantly lack thereof), and to Mike and his great stories.

Huge thanks goes to the rest of the Hadj-Moussa's. None of this would have been possible without the unwavering support and encouragement of my family and parents. You are the best! And don't worry Mum and Dad, I won't tell the others that I am your favourite child. 
DONE. And it's not even 11:59 PM.
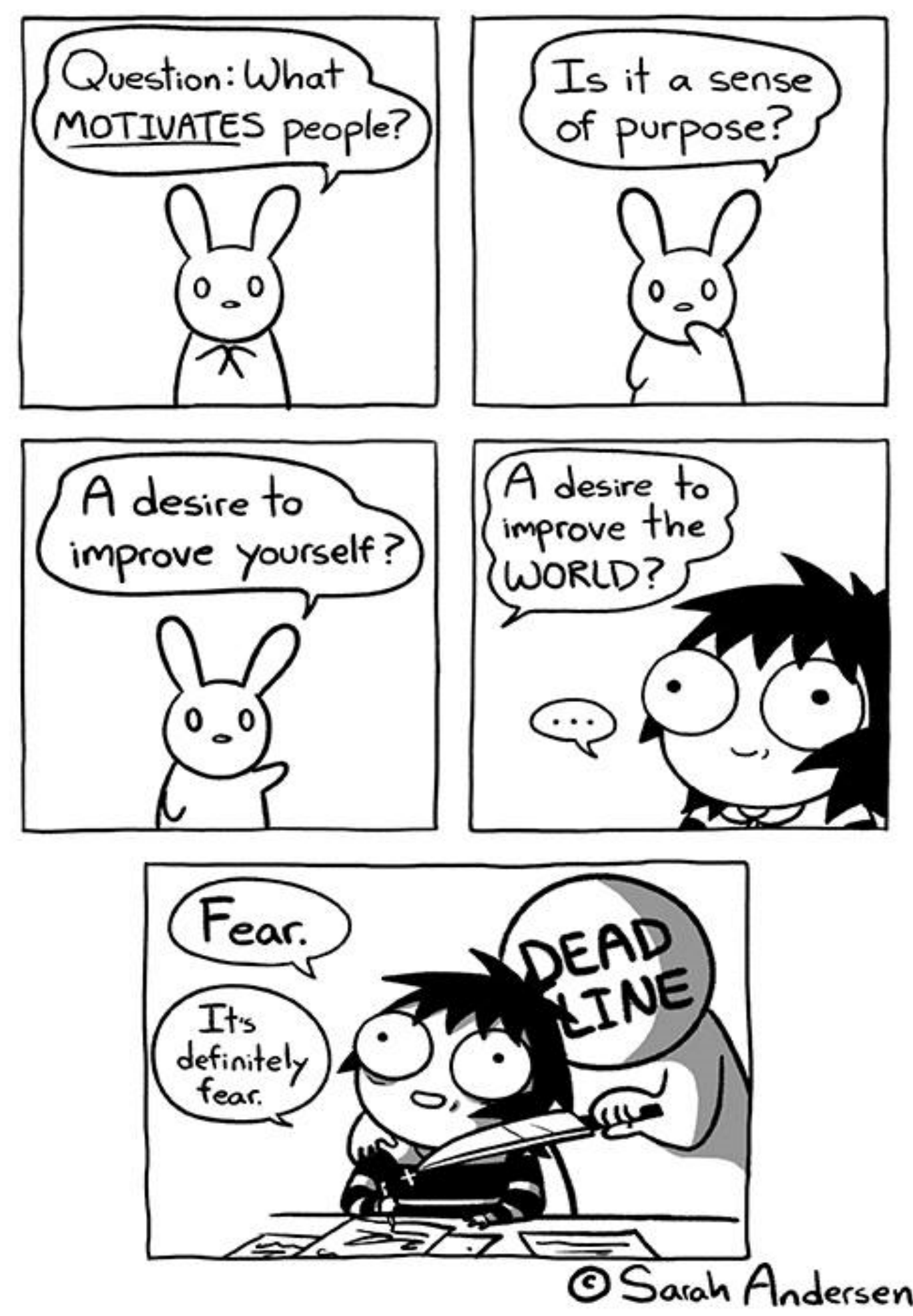

vi 


\section{TABLE OF CONTENTS}

Title Page .................................................................................................................................. i

Acceptance Sheet........................................................................................................................... ii

Abstract............................................................................................................................................ iii

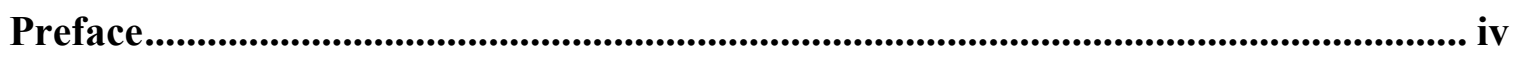

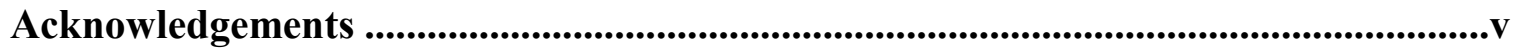

Table of Contents .................................................................................................... vii

List of Abbreviations .................................................................................................................. ix

List of Figures................................................................................................................................ xii

List of Appendices....................................................................................................................... xiii

Chapter 1 - General Introduction.........................................................................................1

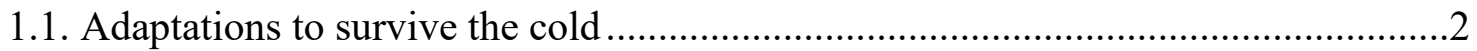

1.2. Rana sylvatica, vertebrate model of natural freeze tolerance ..................................3

1.3. Post-transcriptional regulation via microRNA .......................................................6

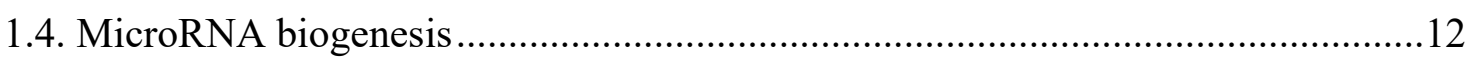

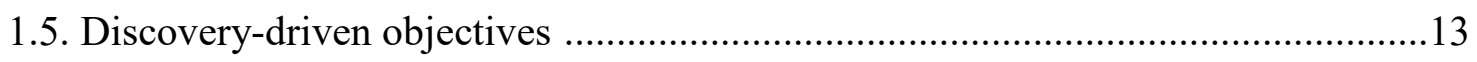

1.5.1. Objective 1: Examine microRNA biogenesis and regulation over a freeze-thaw cycle in wood frog brains ...............................................13

1.5.2. Objective 2: Examine microRNA biogenesis and regulation over a freeze-thaw cycle in wood frog livers................................................14

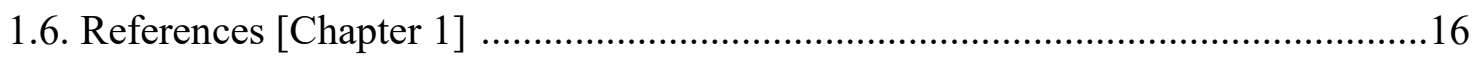

Chapter 2 - General Materials and Methods..................................................................22

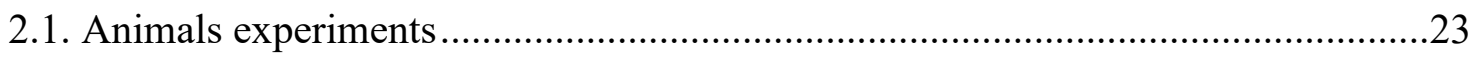

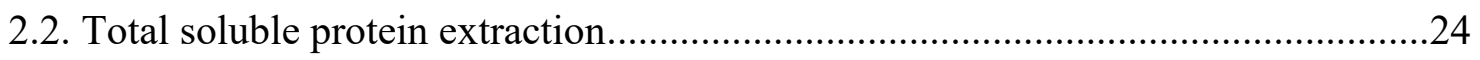

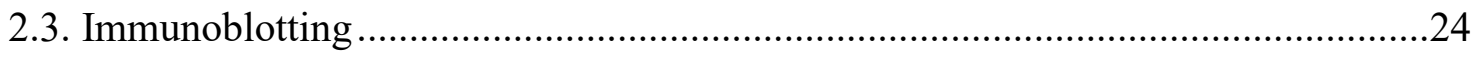

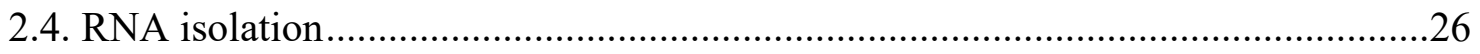

2.5. Polyadenylation and stem-loop reverse transcription ..........................................29

2.6. MicroRNA sequence conservation and primer design............................................. 31

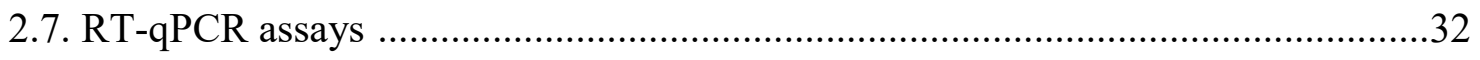

2.8. Bioinformatics microRNA target identification and enrichment .............................33

2.9. Quantification and Statistics ........................................................................... 


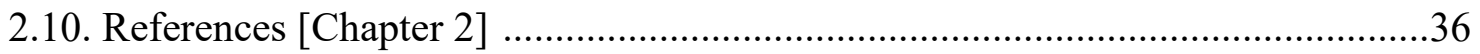

Chapter 3 - Frozen Brain MicroRNAs ..................................................................................37

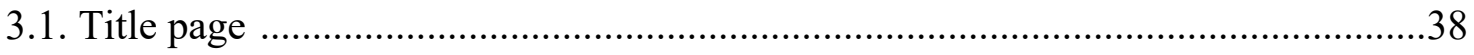

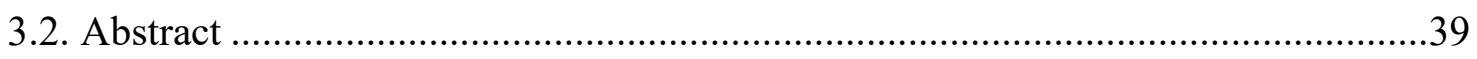

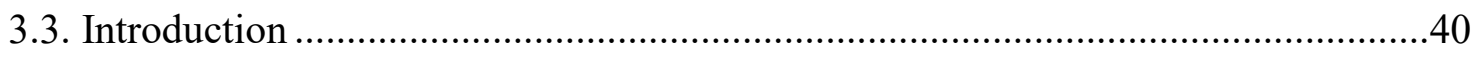

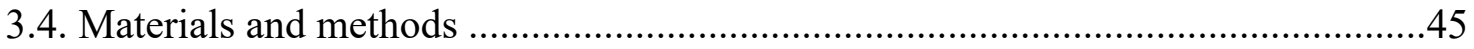

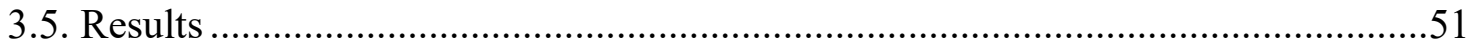

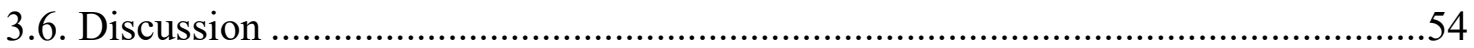

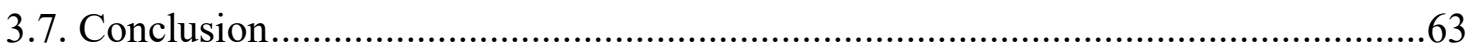

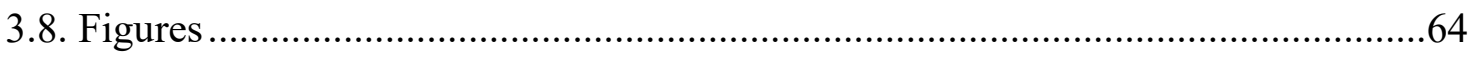

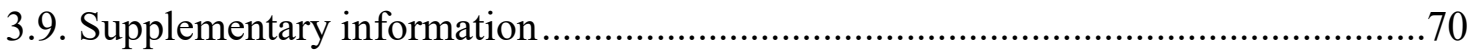

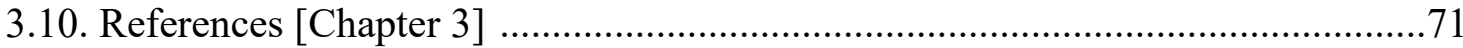

Chapter 4 - Frozen Live MicroRNAs .......................................................................77

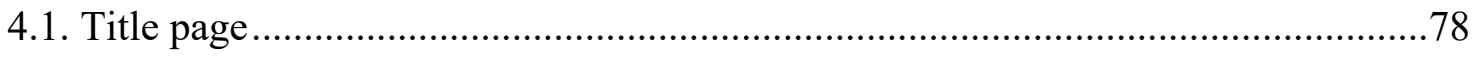

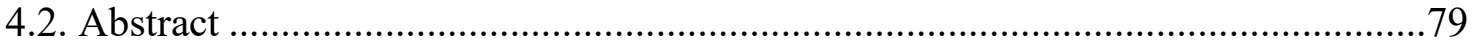

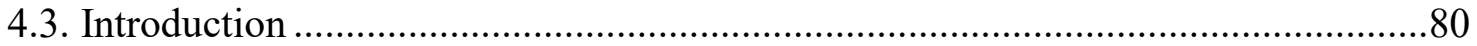

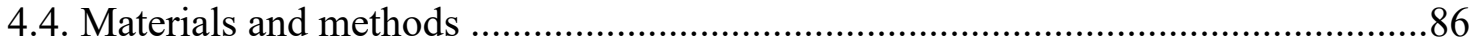

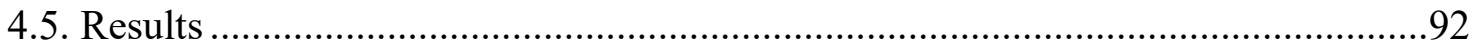

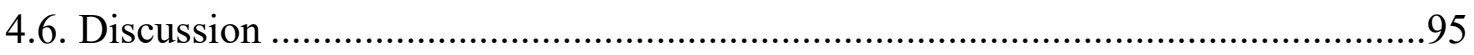

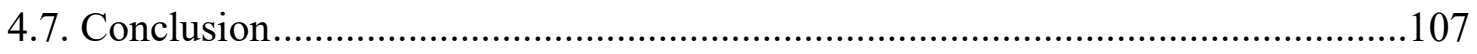

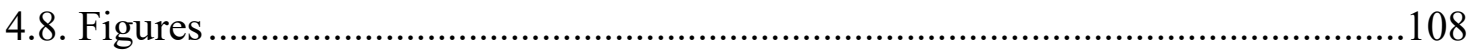

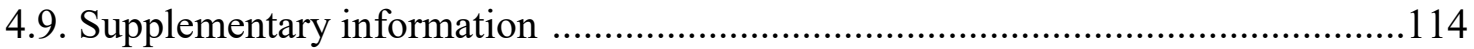

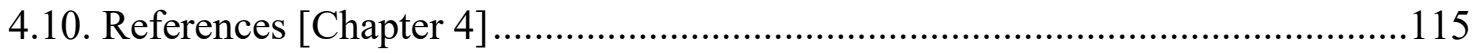

Chapter 5 - General Discussion..............................................................................121

5.1. Wood frogs and freezing ............................................................................122

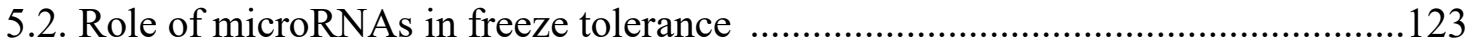

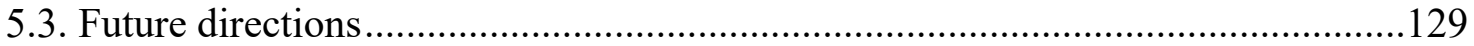

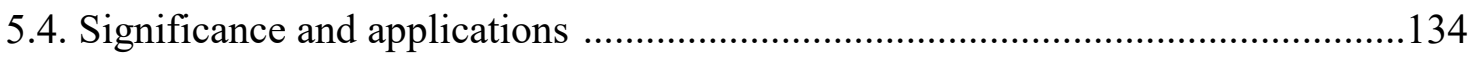

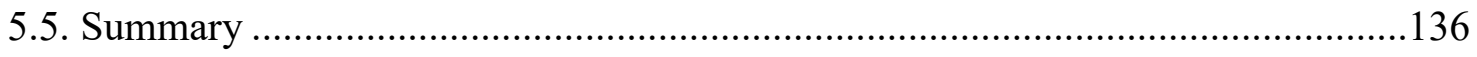

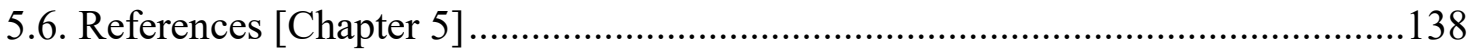

Appendices............................................................................................................141 


\section{LIST OF ABBREVIATIONS}

$\begin{array}{ll}\text { 13LGS } & \text { 13-lined ground squirrel } \\ \text { AGO } & \text { Argonaute } \\ \text { AMPK } & \text { AMP-activated protein kinase } \\ \text { ATP } & \text { Adenosine triphosphate } \\ \text { BCL2 } & \text { B-cell lymphoma } 2 \\ \text { bP } & \text { Base pair } \\ \text { CDK } & \text { Cyclin-dependant kinase } \\ \text { cDNA } & \text { Complementary DNA } \\ \text { DGCR8 } & \text { DiGeorge syndrome critical region gene } 8 \\ \text { DMAP1 } & \text { DNA methyltransferase associated protein 1 } \\ \text { DNA } & \text { Deoxyribonucleic acid } \\ \text { DNMT } & \text { DNA methyltransferase } \\ \text { dNTP } & \text { Deoxynucleotide } \\ \text { EDTA } & \text { Ethylenediaminetetraacetic acid } \\ \text { EGFR } & \text { Epidermal growth factor receptor } \\ \text { FISH } & \text { Fluorescence RNA in situ hybridization } \\ \text { FOXO } & \text { Forkhead box O } \\ \text { FR10 } & \text { Freeze responsive protein clone 10 } \\ \text { FR47 } & \text { Freeze responsive protein clone } 47 \\ \text { GO } & \text { Gene ontology } \\ \text { HRP } & \text { Horseradish peroxidase } \\ \text { INP } & \text { Miver protein clone 16 } \\ \text { Li16 } & \text { Mitogen-activated protein kinases } \\ \text { MAPK } & \text { MCL }\end{array}$




\begin{tabular}{ll} 
miRNA & MicroRNA \\
mRNA & Messenger RNA \\
mTOR & Mechanistic target of rapamycin \\
NADP & Nicotinamide adenine dinucleotide phosphate \\
NGS & Next Generation Sequencing \\
nt & Nucleotide \\
P-body & Processing body \\
PABP & Poly(A)-binding protein \\
PACT & Protein kinase RNA activator \\
PI3K & Phosphoinositide 3-kinase \\
PMSF & Phenylmethylsulfonyl fluoride \\
Pre-miRNA & Precursor microRNA \\
Pri-miRNA & Primary microRNA \\
PTM & Post -translational modifications \\
PVA & Polyvinyl alcohol \\
PVDF & Polyvinylidene fluoride \\
RBP & RNA-binding protein \\
RIP & RNA immunoprecipitation \\
RIP-Seq & RNA immunoprecipitation and Sequencing \\
RISC & RNA-induced silencing complex \\
RNA & Ribonucleic acid \\
RNP & Ribonucleoprotein \\
ROS & Reactive oxygen species \\
RPLP0 & 60S acidic ribosomal protein P0 \\
RT-qPCR & Quantitative real time PCR \\
SDS-PAGE & Sodium dodecyl sulfate polyacrylamide gel electrophoresis \\
\hline
\end{tabular}


SMARCB1

SNAP25

SOD

TBST

TEMED

TGF-ß1

TIA

TRBP

UTR

XIAP

XPO5
SWI/SNF-related matrix-associated actin-dependent regulator of chromatin subfamily B member 1

Synaptosomal-associated protein of $25 \mathrm{kDa}$

Superoxide dismutase

Tris-buffered saline and Tween 20

Tetramethylethylenediamine

Transforming growth factor- $\beta 1$

$\mathrm{T}$-cell restricted intracellular antigen protein

Transactivation response RNA binding protein

Untranslated region

X-linked inhibitor of apoptosis

Exportin-5 


\section{LIST OF FIGURES}

Figure 1.1. Canonical pathway of microRNA biogenesis and processing. 8

Figure 1.2. Temperature influence on miRNA targeting. 11

Figure 2.1. General workflow of microRNA experimetns, data collection, 28 and downstream functional bioinfromaics analyses.

Figure 2.2. Schematic of the polyadenylated microRNA amplification 30 protocol using stem-loop primers.

Figure 3.1. Analysis of miRNA biogenesis pathway protein levels in 64 wood frog brain over a freeze-thaw cycle using immunoblotting.

Figure 3.2. Heatmap of RT-qPCR measured expression levels of $113 \quad 65$ miRNA species examined in $24 \mathrm{~h}$ frozen and $8 \mathrm{~h}$ thawed wood frog brain, relative to control.

Figure 3.3. Functional target enrichment and network clustering of the 66 miRNAs downregulated in the brains of $24 \mathrm{~h}$ frozen wood frogs.

Figure 3.4. Functional target enrichment and network clustering of the miRNAs downregulated in the brains of $8 \mathrm{~h}$ thawed wood frogs.

Figure 4.1. Analysis of miRNA biogenesis pathway protein levels in wood frog liver over a freeze-thaw cycle using immunoblotting.

Figure 4.2. Heatmap of RT-qPCR measured expression levels of 109 miRNA species examined in $24 \mathrm{~h}$ frozen and $8 \mathrm{~h}$ thawed wood frog livers, relative to control.

Figure 4.3. Functional target enrichment and network clustering of the subset of miRNAs upregulated in $24 \mathrm{~h}$ frozen wood frog livers.

Figure 4.4. Functional target enrichment and network clustering of the subset of miRNAs upregulated in $8 \mathrm{~h}$ thawed wood frog livers. 


\section{LIST OF APPENDICES}

APPENDIX A: $\quad$ Publications, Conferences, and Grants 142

$\begin{array}{lll}\text { APPENDIX B: } & \text { Antibody supplier information }\end{array}$

APPENDIX C: $\quad$ Verifying antibody specificity for immunoblotting 149

APPENDIX D: $\quad$ Optimized Immunoblotting conditions 153

APPENDIX E: $\quad$ Primer Sequences 155

APPENDIX F: $\quad$ Bioinformatics workflow for temperature-sensitive $\quad 160$ miRNA target prediction

APPENDIX G: $\quad$ Bioinformatics workflow for discovery of novel 166 species-specific microRNA

$\begin{array}{lll}\text { APPENDIX H: } \quad \text { Novel wood frog specific microRNA sequences } & 177\end{array}$ 
CHAPTER 1

General Introduction 


\subsection{Adaptations to survive the cold}

Many animals are faced with environmental challenges in winter including; food scarcity, short photoperiods, and most importantly, subzero temperatures. Surviving this winter landscape requires a myriad of physiological and molecular adaptations, and while some animals are able to escape the cold by migration, others have adapted unique energy-saving strategies for dealing with the extreme cold. Terrestrial ectotherms have limited options when it comes to dealing with freezing temperatures; some hibernate and seek shelter, others employ active freeze avoidance strategies, while a few have evolved the extreme overwintering solution of freeze tolerance (Storey and Storey, 2013). Despite freezing being lethal for most animals, freezing survival has been documented in species including various soil microfauna, selected intertidal marine invertebrates, many insects, and various ectothermic vertebrates including some turtles, snakes, salamanders and frogs (Ring, 1982; Murphy, 1983; Thomashow, 1999; Costanzo et al., 2008; Holmstrup, 2014; Storey and Storey, 2017). Freeze tolerance is the ability to survive long-term freezing of up to $70 \%$ of total body water as extracellular ice at subzero temperatures experienced naturally in winter hibernacula. This is coupled with the ability to endure the various cellular consequences of freezing, prolonged metabolic rate depression, and the cessation of seemingly vital processes (Storey and Storey, 2017).

Freezing imposes numerous cellular stresses including; anoxia, ischemia/reperfusion, cell dehydration and shrinkage, elevated osmolality, as well as ice-induced mechanical damage. To overcome these freeze-associated challenges, animals have adopted unique strategies such as the use of nonspecific ice nucleators or specialized ice nucleating proteins (INP) to instigate freezing and control ice crystal distribution and crystal growth (Storey and Storey, 
1988). Another adaptation is the accumulation of small molecule cryoprotectants within cells to lower the freezing point of intracellular fluids, counteract osmotic forces, help maintain cell volume, and stabilize macromolecules (Storey and Storey, 2017). Freeze tolerant animals have also evolved mechanisms to circumvent the stresses imposed by the physiological challenges of whole body freezing that include the prolonged cessation of heartbeat, circulation, movement, brain activity, and breathing (Storey and Storey, 2017). Freezing also imposes energetic constraints on animals since the anoxic conditions caused by cessation of heartbeat and breathing forces a shift from aerobic energy production to anaerobic energy processes. To survive these numerous stresses, animals coordinate the global suppression of most metabolic functions along with an overall reduction in ATP turnover and reprioritization of energy use towards basic survival requirements (Storey and Storey, 2013). Indeed, retreating into prolonged hypometabolic states is a common theme that has been observed in other animal adaptations to environmental stress including; hibernation, anoxia tolerance, and estivation (Storey, 2015).

\subsection{Rana sylvatica, vertebrate model of natural freeze tolerance}

The wood frog, Rana sylvatica (also known as Lithobates sylvaticus) is the most wellstudied freeze tolerant vertebrate. Wood frogs have developed multiple mechanisms to aid freezing survival and can endure the accumulation of $65-70 \%$ of total body water as extracellular ice. In their most northern locations in Alaska, wood frogs are able to survive temperatures as low as $-18^{\circ} \mathrm{C}$ for months at a time (Larson et al., 2014). Wood frog natural habitats stretch from the Southern Appalachians to above the Arctic Circle, making them the most northerly-distributed amphibian in North America (Lee-Yaw et al., 2008). When 
in a frozen state, these frogs display no measurable brain activity, no heartbeat, no breathing and cease all skeletal muscle movement (Layne et al., 1989). Yet despite these challenges, the wood frog manages to safely freeze by intricately regulating various molecular systems such that, come spring, it is able to thaw unscathed and resume normal life (Storey and Storey, 2004).

Freezing in wood frogs can be initiated in two main ways: (a) by contact with environmental ice that seeds the freezing of body fluids across the skin, or (b) by the action of ice nucleators including non-specific nucleators such as bacteria on the skin or via the action of specifically-synthesized ice nucleating proteins (INPs) (Storey and Storey, 1985; Lee and Costanzo 1998; Zachariassen and Kristiansen 2000). This occurs at temperatures near the freezing point of body fluids, between $-0.5^{\circ} \mathrm{C}$ and $-3^{\circ} \mathrm{C}$. Freezing at such relatively high subzero temperatures allows for a slow rate of ice formation and minimizes the instantaneous ice surge (instant conversion of water to ice) that occurs if animals supercool extensively before freezing. This provides the frog with sufficient time to adjust their metabolism and synthesize and distribute cryoprotectant as the freezing front moves through the body (Storey, 1997). The initial ice nucleation event triggers a $\beta$-adrenergic signaling cascade (the fight-or-flight response) which initiates various molecular freeze tolerance responses that are highlighted below (Storey and Storey, 1996).

Within 1-2 minutes of ice nucleation, the liver begins to catabolize large quantities of glycogen and synthesize glucose resulting in the rapid increase of glucose levels from 1-2 $\mathrm{mM}$ in active frogs to $\sim 300 \mathrm{mM}$ in frozen frogs (Storey and Storey 1984; Storey et al., 1992). Wood frogs rely on the synthesis of the colligative cryoprotectant glucose as their main line of defense as glucose helps limit the amount of water that is converted into 
extracellular ice, minimize cell volume reduction, and help stabilize macromolecules (Costanzo et al., 1993). The liver rapidly exports glucose into the bloodstream where it is transported to the rest of the body before the heart stops beating and blood circulation ceases (Storey and Storey 1986). To facilitate this rapid synthesis and distribution of large quantities of glucose the wood frog prepares itself by building up large glycogen reserves and enhancing liver glucose transporter capacity in liver during the summer-autumn months (King et al. 1993, 1995). In addition, the nucleation event also triggers cardioacceleration that is believed to facilitate the rapid distribution of glucose (Layne et al., 1989).

When frozen, frogs must rely on their internal fuel reserves- this poses various restrictions on energy availability. To overcome this, the wood frog lowers it metabolic rate and transitions from oxidative phosphorylation to anaerobic glycolysis due to the lack of oxygen availability during freezing. Measurements in other hypometabolic animals show that metabolic rate decreases to $1-30 \%$ of normal resting rate (Storey and Storey, 2017). To endure prolonged freezing, the frog globally suppresses various energy-expensive cell processes such as DNA replication, transcription, translation, and numerous other nonessential energy-expensive processes. This is coordinated by many signal transduction cascades and biochemical regulatory mechanisms (Storey and Storey, 2004, 2007). Yet, despite the overall reduction in ATP turnover, there is a reprioritization of energy use towards select pathways required for basic cellular survival and for defending against freeze-associated damages (Storey and Storey, 2013). Indeed, against this background of global suppression of transcription and translation, a variety of freeze-upregulated genes have been identified and act to elevate protective proteins over freeze-thaw cycles and 
enhance pro-survival pathways (Cai and Storey, 1997; McNally et al., 2002; Storey and Storey, 2004). Findings from recent years have demonstrated that freezing in the wood frog results in the induction of $\mathrm{p} 38$ MAPK, AMPK, and Akt signal transduction pathways (Greenway and Storey, 2000; Rider, 2006; Zhang and Storey, 2013). This is in addition to the modulation of key enzymes involved in energetics and antioxidant defenses by posttranslational modifications (Dieni and Storey, 2008; Dieni et al., 2012; Dawson et al., 2015; Dawson and Storey, 2016).

\subsection{Post-transcriptional regulation of stress responses via microRNAs}

The reduction in ATP turnover and reprioritization of energy usage that occurs in the frozen state implies that quick, reversible, and energetically inexpensive regulatory mechanisms that are triggered by extracellular stimuli are responsible for adapting cells for survival. Indeed, studies have found that survival in this complex frozen state is orchestrated by various molecular mechanisms, including; reversible protein phosphorylation of many enzymes and functional proteins as well as transcription and translation factors, the use of epigenetic mechanisms to directly modify DNA and histone proteins, and posttranscriptional controls on mRNA through the action of microRNAs (miRNAs) (Storey, 2015). MicroRNAs are proving to be master regulators of virtually all cell processes with broad controls over many activities including the cell cycle, signal transduction pathways, and energy metabolism, among others (Leung and Sharp, 2010; Biggar and Storey, 2011). While this large group of highly-conserved, short, non-coding RNA transcripts ( $22 \mathrm{nt})$ comprises just $1-2 \%$ of the number of protein-coding genes, recent computational predictions indicate that they target more than $60 \%$ of protein-coding genes in humans, and influence almost every aspect of biological function (Bartel, 2009; Ebert and Sharp, 2012). 
Indeed, depending on the miRNA mode of action for each mRNA target, miRNAs can effectively mold the composition of the transcriptome during stress.

MicroRNAs function by associating with the RNA-induced silencing complex (RISC), directing the complex to the appropriate mRNA transcript(s), binding with partial or perfect complementarity to mRNA transcripts, and ultimately resulting in the translational repression or degradation of the bound mRNA (Bartel, 2004) (Figure 1.1.). The $5^{\prime}$ seed region of the guide strand, involving nucleotides two to eight, directs RISC activity towards target mRNAs with complementary motifs in their $3^{\prime}$ UTR (Huang et al., 2011). The brevity of the seed region is what allows miRNAs to exert their broad controls, wherein (a) a single miRNA can regulate multiple mRNA transcripts, and (b) one mRNA transcript may be subject to regulation by multiple miRNAs (Bartel, 2004). Once bound, the degree of complementarity between the miRNA and mRNA transcript dictates the fate of the mRNA transcript, providing another level of control. Imperfect complementarity causes mRNA transcripts to be sequestered to cytoplasmic loci such as P-bodies and stress granules, where they are subject to translational repression; in contrast, perfect complementarity triggers mRNA degradation by Argonaute endonucleases (Bartel, 2004). A transient outcome of miRNA targeting is accelerated target mRNA deadenylation which leads to mRNA destabilization and rapid mRNA decay (Wu et al., 2006). The importance of miRNA regulation can be observed in the high degree of conservation that miRNA sequences display between distant vertebrate species. Indeed, this is also emphasized by the conservation of corresponding target mRNA 3'UTR binding motifs that are $90-100 \%$ conserved, despite the overall low conservation generally observed in 3'UTR regions (Friedman et al., 2009; Lewis et al., 2009). 


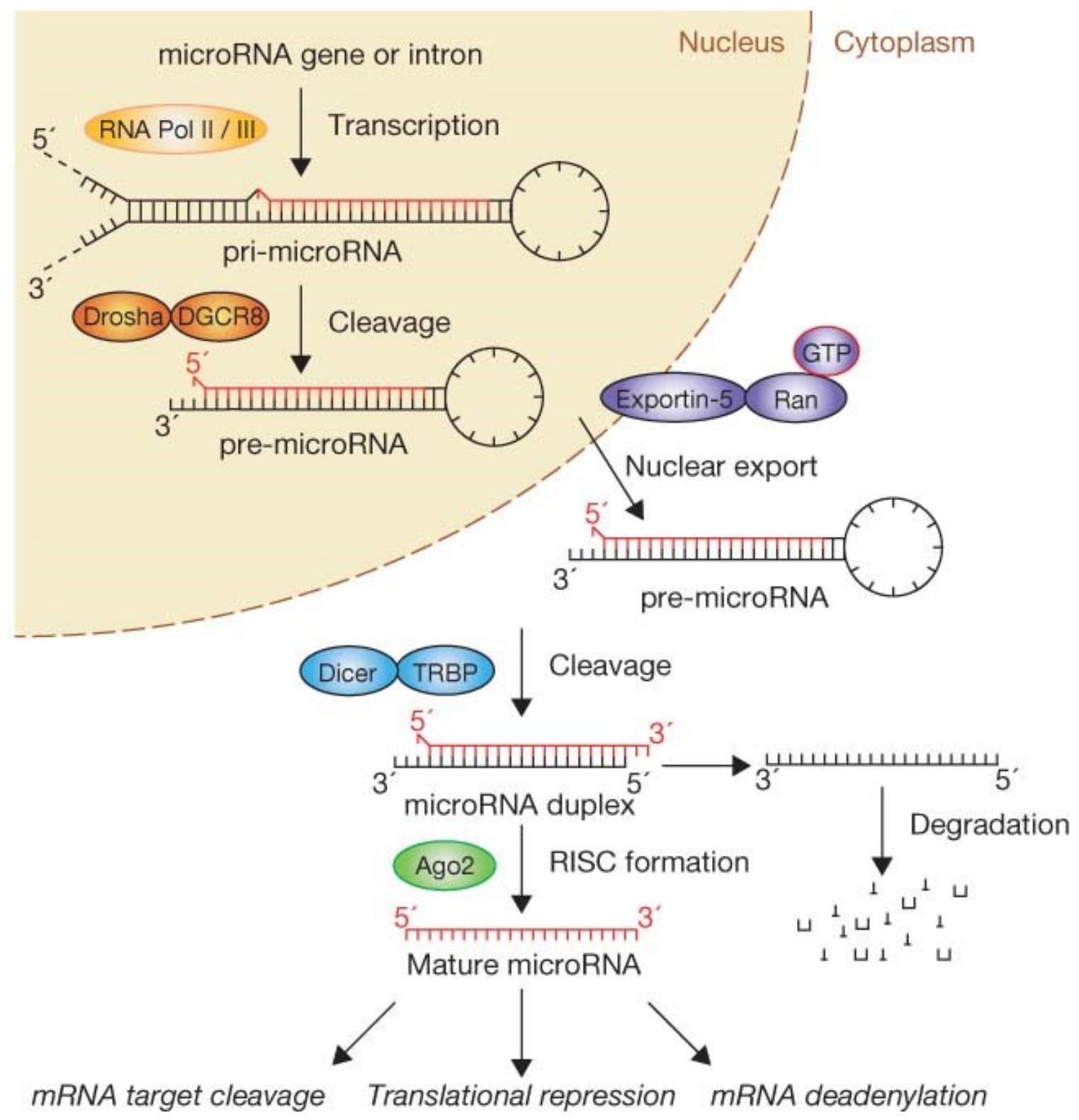

Figure 1.1. Canonical pathway of microRNA biogenesis and processing. This schematic includes the main biogenesis factors involved in 'linear' canonical microRNA maturation. Figure from (Winter et al., 2009). 
Since the initial discovery of miRNAs in Caenorhabditis elegans in 1993 (Lee et al., 1993), a growing number of studies in diverse stress-tolerant animals have shown that miRNAs involved in regulating cell survival are under dynamic regulation in response to environmental stress (Biggar and Storey, 2015a). MicroRNAs have been implicated as a regulatory mechanism in many situations of biochemical adaptation and/or transitions into hypometabolic states including; hibernation (Morin et al., 2008; Kornfeld et al., 2012; Wu et al., 2014, 2016; Hadj-Moussa et al., 2016), turtle and invertebrate anoxia tolerance (Biggar and Storey 2012; Biggar et al., 2012, 2015a), sea cucumber and frog estivation (Chen and Storey, 2014; Chen et al., 2013; Wu et al., 2013; Luu and Storey, 2015), freezeavoidance in insects (Lyons et al., 2015), and freeze tolerance in insects, frogs, and turtles (Biggar et al., 2009; Courteau et al., 2012; Lyons et al., 2015a; Bansal et al., 2016). Several investigations of miRNA involvement in cold-tolerant vertebrates have identified a subset of cold-associated miRNAs termed 'Cryo-miRs' that have been shown to be involved in muscle atrophy prevention, cell cycle regulation, and glucose and lipid metabolism (Lyons et al., 2013). A recent study on wood frog heart and skeletal muscle demonstrated tissuespecific differential expression of miRNAs involved in maintaining cell structure, muscle contraction, and reversible phosphorylation over the freeze-thaw cycle (Bansal et al., 2016). Taken together, these studies suggest that miRNAs play specific freeze and coldresponsive roles to facilitate the cryoprotective and metabolic changes necessary for successful freeze-thaw.

The thermodynamics of miRNA-mRNA interactions can be influenced by factors in the cellular environment, such as temperature ( $\mathrm{Wu}$ et al., 2002) (Figure 1.2.). This is of particular interest when examining ectothermic species that exhibit large fluctuations in 
body temperature. RNA strands hybridize to each other through hydrogen bonding, with two hydrogen bonds between Adensoine and Uracil, and three hydrogen bonds between Guanosine and Cytosine, in addition to surrounding hydrophobic interactions between nitrogenous bases that facilitate the formation of complex secondary structures. When body temperatures decrease, this favors miRNA binding to mRNA due to a decrease in the free energy required for the formation of the RNA duplex (Wu et al., 2002; Biggar and Storey, 2014). These cold-influenced interactions are a result of the differential strength of bondsas temperature changes, hydrophobic and hydrogen bonds have opposite thermal profiles. At low temperatures, the decrease in kinetic energy strengthens hydrogen bonds and contrastingly results in a decrease in hydrophobic interactions. As a result, events such as freezing have been shown to lead to significant changes in miRNA functionality, increasing the number of mRNA transcripts that can be binding partners of individual miRNAs, and thereby increase/change the number/type of mRNA transcripts that can be regulated by individual miRNAs (Biggar and Storey, 2015a, 2015b). It should be noted that temperature is not the only factor that affects miRNA binding thermodynamics and duplex formation, other factors include; intracellular ionic strength, protein interactions, phospholipids present in membranes, and neighbouring small molecular weight metabolites such as cryoprotectant glucose. 

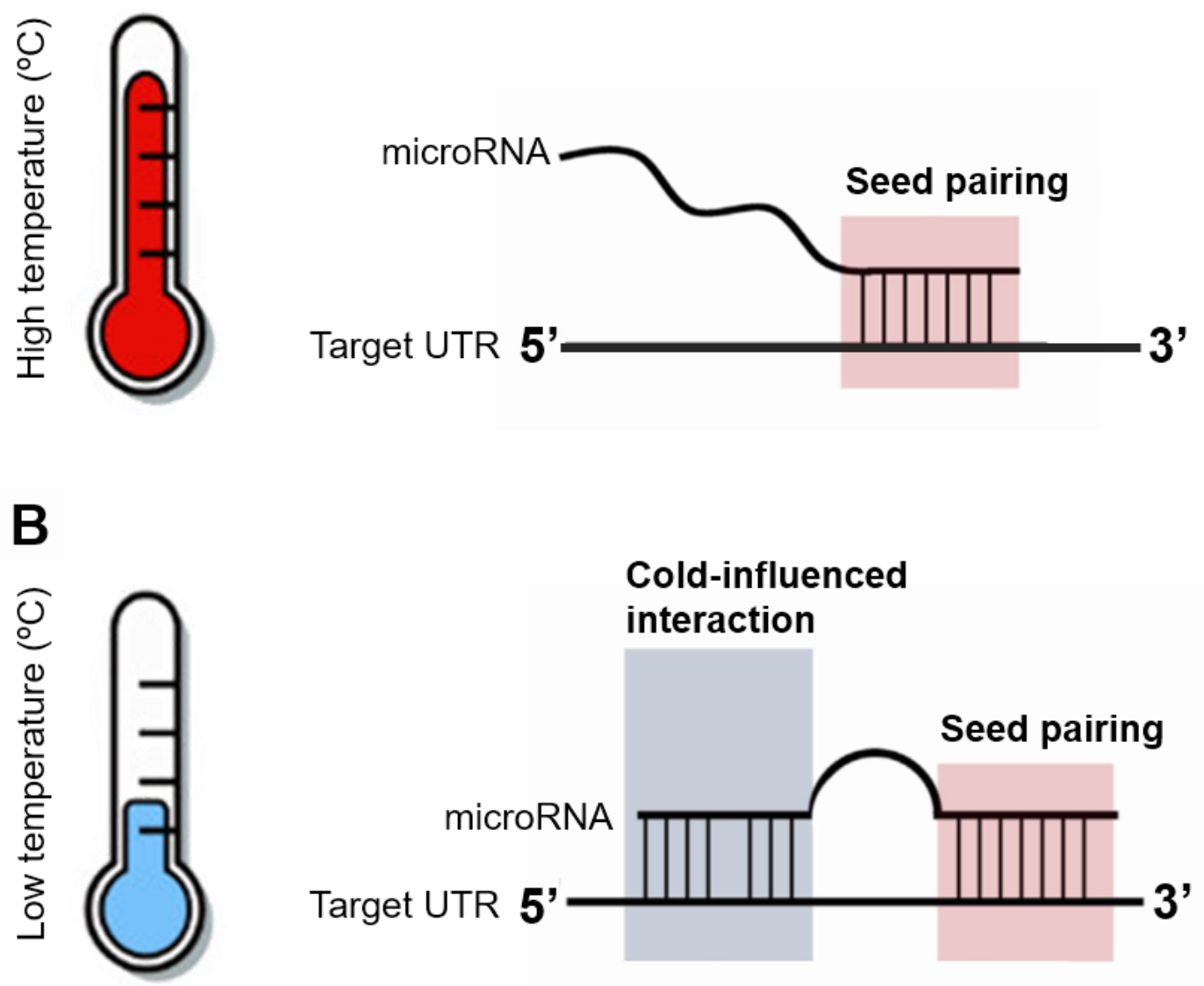

Figure 1.2. Temperature influence on miRNA targeting. Proposed temperature influence on stabilizing miRNA interactions with target mRNA. [A] Effect of high temperature on miRNA-mRNA binding, [B] Effect of low temperature on miRNA-mRNA binding. Modified from (Biggar and Storey, 2015b). 


\subsection{MicroRNA biogenesis}

Although miRNAs have emerged as master regulators of nearly all biological functions, including stress responses, relatively little work has gone into investigating the regulation of miRNA biogenesis machinery under stress conditions. Indeed, miRNA biogenesis pathways have been shown to be under tight spatial and temporal control, suggesting that this might also be the case during stress responses (Ha and Kim, 2014). MicroRNAs are initially transcribed by RNA polymerase II into long secondary hairpin structures known as primary miRNAs (pri-miRNAs) (Huang et al., 2011). The maturation process begins with the cleavage of the $5^{\prime}$ and $3^{\prime}$ ends by the RNase III endonuclease DROSHA and its binding partner the double-stranded RNA-binding protein DiGeorge syndrome critical region gene 8 (DGCR8) to form a $\sim 60-70$ nt precursor miRNA (pre-miRNA) (Figure 1.1.). Current processing models suggest that DGCR8 directs DROSHA to a specific cleavage site $\sim 11 \mathrm{bp}$ from the junction of the stem of the hairpin by recognizing the pri-miRNA ssRNA-dsRNA junction (Han et al., 2004, 2006; Lee et al., 2002).

The pre-miRNA then complexes with the nucleocytoplasmic transporter factor EXPORTIN-5 and RAN-GTP to protect against nuclear degradation and facilitate nuclear export into the cytoplasm for further processing (Bohnsack et al., 2004). At this stage, cytoplasmic maturation involves the cleavage of the pre-miRNA stem-loop by the RNase III endonuclease DICER to create a ds-miRNA duplex (MacFarlane and Murphy, 2010) (Figure 1.1.). DICER is associated with transactivating response RNA-binding protein (TRBP) that modulates the processing efficiency of select pre-miRNAs and tunes the length of mature miRNAs (Fukunaga et al., 2012; Lee and Doudna, 2012). The DICERTRBP complex is further complexed by the ARGONAUTE family of endonucleases and 
by protein activator of protein kinase R (PACT), a third dsRNA binding protein that currently plays an unknown role in miRNA biogenesis (Ha and Kim, 2014). The mature miRNA guide strand is then incorporated into the effector RISC, where miRNAs use basepairing to negatively regulate the expression of target mRNAs (Bartel, 2004).

\subsection{Discovery-driven objectives}

1.5.1. Objective 1: Examine microRNA biogenesis and regulation over the freeze-thaw cycle in wood frog brain

The brain's persistent flow of information and adaptations to environmental stimuli plays a pivotal role in the facilitation of stress responses and prolonged hypometabolic periods (Storey and Storey, 2007). While it has been reported that frozen wood frog brains display no detectable brain activity and both visual observations and magnetic resonance imaging have shown ice accumulation in brain ventricles, little other physiological work has been

done on freeze tolerant brains (Storey and Storey, 2017). Previous biochemical and molecular studies on frozen frog brains have revealed various cytoprotective "activations" of maintenance and freeze protective pathways that promote tissue survival (Aguilar et al., 2016; Cai and Storey, 1997; Greenway and Storey, 2000; Sullivan and Storey, 2012). The rapid, reversible, and energetically inexpensive nature of miRNA control renders these small molecules excellent candidates for coordinating the necessary neuroprotection of intricate neural networks and facilitating freeze survival. 
To that end, Chapter 3 explores how the miRNA biogenesis pathway is regulated in the wood frog brain by measuring the levels key proteins in the biogenesis pathway that include; DROSHA, DGCR8, EXPORTIN-5, RAN, DICER, TRBP, PACT, AGO1, and AGO2. This was followed up with a large-scale profile of miRNA expression levels over the freeze-thaw cycle. Bioinformatics tools were then used to identify the targets of the differentially expressed miRNAs and to elucidate the roles of the stress-responsive responsive miRNAs in brain freeze tolerance.

\subsubsection{Objective 2: Examine microRNA biogenesis and regulation over a freeze-thaw cycle} in wood frog liver

Changes to liver metabolism are central to freeze survival. Wood frog liver is responsible for generating the cryoprotectant glucose and exporting it to the whole body. The liver also produces various proteins essential for survival, such as fibrinogen that protects against and repairs minor ice damage injuries, and novel proteins FR10, FR47, and Li16 that promote freeze survival (Storey and Storey, 1986; Cai and Storey, 1997). Indeed, the liver is the last organ to freeze and the first one to thaw, emphasizing its vital role in facilitating successful freezing and its high concentration of glucose cryoprotectant (Rubinsky et al., 1994). The regulatory mechanisms that govern the tight regulation of liver functions during a time of global metabolic rate depression are not well characterized and one promising candidate control mechanism is the specific and reversible action of miRNAs. 
Chapter 4 investigates the effects of freeze-thaw on miRNA biogenesis by measuring the protein levels of the main miRNA biogenesis protein members in liver; DROSHA, DGCR8, EXPORTIN-5, RAN, DICER, TRBP, PACT, AGO1, and AGO2. The regulation of liver miRNA expression levels was then measured over the freeze-thaw cycle. The biological functions and mRNA targets of the freeze- and thaw-responsive miRNAs were then bioinformatically identified and their role in hepatic freeze tolerance was determined. 


\section{REFERENCES: [CHAPTER 1]}

Aguilar, O. A., Hadj-Moussa, H., \& Storey, K. B. (2016). Regulation of SMAD transcription factors during freezing in the freeze tolerant wood frog, Rana sylvatica. Comparative Biochemistry and Physiology Part B: Biochemistry and Molecular Biology, 201, 64-71.

Bansal, S., Luu, B. E., \& Storey, K. B. (2016). MicroRNA regulation in heart and skeletal muscle over the freeze-thaw cycle in the freeze tolerant wood frog. Journal of Comparative Physiology B, 186(2), 229-241.

Bartel, D. P. (2004). MicroRNAs: genomics, biogenesis, mechanism, and function. Cell, $116(2), 281-297$.

Bartel, D. P. (2009). MicroRNAs: target recognition and regulatory functions. Cell, 136(2), 215-233.

Biggar, K. K., Dubuc, A., \& Storey, K. (2009). MicroRNA regulation below zero: differential expression of miRNA-21 and miRNA-16 during freezing in wood frogs. Cryobiology, 59(3), 317-321.

Biggar, K. K., \& Storey, K. B. (2010). The emerging roles of microRNAs in the molecular responses of metabolic rate depression. Journal of molecular cell biology, 3(3), 167-175.

Biggar, K. K., \& Storey, K. B. (2012). Evidence for cell cycle suppression and microRNA regulation of cyclin D1 during anoxia exposure in turtles. Cell Cycle, 11(9), 1705-1713.

Biggar, K. K., Kornfeld, S. F., Maistrovski, Y., \& Storey, K. B. (2012). MicroRNA regulation in extreme environments: differential expression of microRNAs in the intertidal snail Littorina littorea during extended periods of freezing and anoxia. Genomics, proteomics \& bioinformatics, 10(5), 302-309.

Biggar, K. K., \& Storey, K. B. (2014). Insight into temperature-dependent microRNA function in mammalian hibernators: perspectives on cold-influenced microRNA/target interaction. Temperature, 1(2), 84-86.

Biggar, K. K., \& Storey, K. B. (2015a). Insight into post-transcriptional gene regulation: stress-responsive microRNAs and their role in the environmental stress survival of tolerant animals. Journal of Experimental Biology, 218(9), 1281-1289.

Biggar, K. K., \& Storey, K. B. (2015b). Low-temperature microRNA expression in the painted turtle, Chrysemys picta during freezing stress. FEBS letters, 589(23), 36653670 .

Bohnsack, M. T., Czaplinski, K., \& Gorlich, D. (2004). Exportin 5 is a RanGTPdependent dsRNA-binding protein that mediates nuclear export of pre-miRNAs. $R N A, 10(2), 185-191$.

Cai, Q., \& Storey, K. B. (1997). Freezing-induced genes in wood frog (Rana sylvatica): fibrinogen upregulation by freezing and dehydration. American Journal of 
Physiology-Regulatory, Integrative and Comparative Physiology, 272(5), R1480R1492.

Chen, M., \& Storey, K. B. (2014). Large-scale identification and comparative analysis of miRNA expression profile in the respiratory tree of the sea cucumber Apostichopus japonicus during aestivation. Marine genomics, 13, 39-44.

Chen, M., Zhang, X., Liu, J., \& Storey, K. B. (2013). High-throughput sequencing reveals differential expression of miRNAs in intestine from sea cucumber during aestivation. PLoS One, 8(10), e76120.

Costanzo, J. P., Lee, R. E., \& Lortz, P. H. (1993). Glucose concentration regulates freeze tolerance in the wood frog Rana sylvatica. Journal of Experimental Biology, 181(1), 245-255.

Costanzo, J. P., Lee, R. E., \& Ultsch, G. R. (2008). Physiological ecology of overwintering in hatchling turtles. Journal of Experimental Zoology Part A: Ecological Genetics and Physiology, 309(6), 297-379.

Courteau, L. A., \& Storey, K. B. (2012). Differential expression of microRNA species in a freeze tolerant insect, Eurosta solidaginis. Cryobiology, 65(3), 210-214.

Dawson, N. J., \& Storey, K. B. (2016). A hydrogen peroxide safety valve: The reversible phosphorylation of catalase from the freeze-tolerant North American wood frog, Rana sylvatica. Biochimica et Biophysica Acta (BBA)-General Subjects, 1860(3), 476-485.

Dawson, N. J., Katzenback, B. A., \& Storey, K. B. (2015). Free-radical first responders: the characterization of CuZnSOD and MnSOD regulation during freezing of the freeze-tolerant North American wood frog, Rana sylvatica. Biochimica et Biophysica Acta (BBA)-General Subjects, 1850(1), 97-106.

Dieni, C. A., \& Storey, K. B. (2008). Regulation of 5'-adenosine monophosphate deaminase in the freeze tolerant wood frog, Rana sylvatica. BMC biochemistry, 9(1), 12.

Dieni, C. A., Bouffard, M. C., \& Storey, K. B. (2012). Glycogen synthase kinase-3: cryoprotection and glycogen metabolism in the freeze-tolerant wood frog. Journal of Experimental Biology, 215(3), 543-551.

Ebert, M. S., \& Sharp, P. A. (2012). Roles for microRNAs in conferring robustness to biological processes. Cell, 149(3), 515-524.

Friedman, R. C., Farh, K. K. H., Burge, C. B., \& Bartel, D. P. (2009). Most mammalian mRNAs are conserved targets of microRNAs. Genome research, 19(1), 92-105.

Fukunaga, R., Han, B. W., Hung, J. H., Xu, J., Weng, Z., \& Zamore, P. D. (2012). Dicer partner proteins tune the length of mature miRNAs in flies and mammals. Cell, 151(3), 533-546. 
Greenway, S. C., \& Storey, K. B. (2000). Activation of mitogen-activated protein kinases during natural freezing and thawing in the wood frog. Molecular and cellular biochemistry, 209(1), 29-37.

Ha, M., \& Kim, V. N. (2014). Regulation of microRNA biogenesis. Nature reviews Molecular cell biology, 15(8), 509-524.

Hadj-Moussa, H., Moggridge, J. A., Luu, B. E., Quintero-Galvis, J. F., Gaitán-Espitia, J. D., Nespolo, R. F., \& Storey, K. B. (2016). The hibernating South American marsupial, Dromiciops gliroides, displays torpor-sensitive microRNA expression patterns. Scientific reports, 6(1), 25627.

Han, J., Lee, Y., Yeom, K. H., Nam, J. W., Heo, I., Rhee, J. K., ... \& Kim, V. N. (2006). Molecular basis for the recognition of primary microRNAs by the Drosha-DGCR 8 complex. Cell, 125(5), 887-901.

Han, J., Lee, Y., Yeom, K. H., Kim, Y. K., Jin, H., \& Kim, V. N. (2004). The DroshaDGCR 8 complex in primary microRNA processing. Genes \& development, 18(24), 3016-3027.

Holmstrup, M. (2014). The ins and outs of water dynamics in cold tolerant soil invertebrates. Journal of thermal biology, 45, 117-123.

Huang, Y., Shen, X. J., Zou, Q., Wang, S. P., Tang, S. M., \& Zhang, G. Z. (2011). Biological functions of microRNAs: a review. Journal of physiology and biochemistry, 67(1), 129-139.

King, P. A., Rosholt, M. N., \& Storey, K. B. (1993). Adaptations of plasma membrane glucose transport facilitate cryoprotectant distribution in freeze-tolerant frogs. American Journal of Physiology-Regulatory, Integrative and Comparative Physiology, 265(5), R1036-R1042.

King, P. A., Rosholt, M. N., \& Storey, K. B. (1995). Seasonal changes in plasma membrane glucose transporters enhance cryoprotectant distribution in the freezetolerant wood frog. Canadian journal of zoology, 73(1), 1-9.

Kornfeld, S. F., Biggar, K. K., \& Storey, K. B. (2012). Differential expression of mature microRNAs involved in muscle maintenance of hibernating little brown bats, Myotis lucifugus: a model of muscle atrophy resistance. Genomics, proteomics \& bioinformatics, 10(5), 295-301.

Larson, D. J., Middle, L., Vu, H., Zhang, W., Serianni, A. S., Duman, J., \& Barnes, B. M. (2014). Wood frog adaptations to overwintering in Alaska: new limits to freezing tolerance. Journal of Experimental Biology, 217(12), 2193-2200.

Layne, J. R., Lee, R. E., \& Heil, T. L. (1989). Freezing-induced changes in the heart rate of wood frogs (Rana sylvatica). American Journal of Physiology-Regulatory, Integrative and Comparative Physiology, 257(5), R1046-R1049.

Lee, H. Y., \& Doudna, J. A. (2012). TRBP alters human precursor microRNA processing in vitro. $R N A, 18(11), 2012-2019$. 
Lee Jr, R. E., \& Costanzo, J. P. (1998). Biological ice nucleation and ice distribution in cold-hardy ectothermic animals. Annual Review of Physiology, 60(1), 55-72.

Lee, R. C., Feinbaum, R. L., \& Ambros, V. (1993). The C. elegans heterochronic gene lin-4 encodes small RNAs with antisense complementarity to lin-14. Cell, 75(5), 843-854.

Lee, Y., Jeon, K., Lee, J. T., Kim, S., \& Kim, V. N. (2002). MicroRNA maturation: stepwise processing and subcellular localization. The EMBO journal, 21(17), 46634670 .

Lee-Yaw, J. A., Irwin, J. T., \& Green, D. M. (2008). Postglacial range expansion from northern refugia by the wood frog, Rana sylvatica. Molecular Ecology, 17(3), 867-884.

Leung, A. K., \& Sharp, P. A. (2010). MicroRNA functions in stress responses. Molecular Lewis, B. P., Burge, C. B., \& Bartel, D. P. (2005). Conserved seed pairing, often flanked by adenosines, indicates that thousands of human genes are microRNA targets. cell, 120(1), 15-20. Cell, 40(2), 205-215.

Lewis, B. P., Burge, C. B., \& Bartel, D. P. (2005). Conserved seed pairing, often flanked by adenosines, indicates that thousands of human genes are microRNA targets. Cell, 120(1), 15-20.

Luu, B. E., \& Storey, K. B. (2015). Dehydration triggers differential microRNA expression in Xenopus laevis brain. Gene, 573(1), 64-69.

Lyons, P. J., Lang-Ouellette, D., \& Morin, P. J. (2013). CryomiRs: towards the identification of a cold-associated family of microRNAs. Comparative Biochemistry and Physiology Part D: Genomics and Proteomics, 8(4), 358-364.

Lyons, P. J., Storey, K. B., \& Morin, P. J. (2015a). Expression of miRNAs in response to freezing and anoxia stresses in the freeze tolerant fly Eurosta solidaginis. Cryobiology, 71(1), 97-102.

Lyons, P. J., Crapoulet, N., Storey, K. B., \& Morin, P. J. (2015b). Identification and profiling of miRNAs in the freeze-avoiding gall moth Epiblema scudderiana via next-generation sequencing. Molecular and cellular biochemistry, 410(1-2), 155163.

MacFarlane, L. A., \& R Murphy, P. (2010). MicroRNA: biogenesis, function and role in cancer. Current genomics, 11(7), 537-561.

McNally, J. D., Wu, S. B., Sturgeon, C. M., \& Storey, K. B. (2002). Identification and characterization of a novel freezing inducible gene, li16, in the wood frog Rana sylvatica. The FASEB Journal, 16(8), 902-904.

Murphy, D. J. (1983). Freezing resistance in intertidal invertebrates. Annual review of physiology, 45(1), 289-299.

Morin, P. J., A., \& Storey, K. B. (2008). Differential expression of microRNA species in organs of hibernating ground squirrels: a role in translational suppression during 
torpor. Biochimica et Biophysica Acta (BBA)-Gene Regulatory Mechanisms, 1779(10), 628-633.

Rider, M. H. (2006). The ubiquitin-associated domain of AMPK-related protein kinases allows LKB1-induced phosphorylation and activation. Biochemical Journal, 394(3), e7.

Ring, R. A. (1982). Freezing-tolerant insects with low supercooling points. Comparative Biochemistry and Physiology Part A: Physiology, 73(4), 605-612.

Rubinsky, B., Wong, S. T., Hong, J. S., Gilbert, J., Roos, M., \& Storey, K. B. (1994). 1H magnetic resonance imaging of freezing and thawing in freeze-tolerant frogs. American Journal of Physiology-Regulatory, Integrative and Comparative Physiology, 266(6), R1771-R1777.

Storey, K. B., \& Storey, J. M. (1984). Biochemical adaption for freezing tolerance in the wood frog, Rana sylvatica. Journal of Comparative Physiology B: Biochemical, Systemic, and Environmental Physiology, 155(1), 29-36.

Storey, J. M., \& Storey, K. B. (1985a). Triggering of cryoprotectant synthesis by the initiation of ice nucleation in the freeze tolerant frog, Rana sylvatica. Journal of Comparative Physiology B, 156(2), 191-195.

Storey, K. B., \& Storey, J. M. (1986). Freeze tolerant frogs: cryoprotectants and tissue metabolism during freeze-thaw cycles. Canadian Journal of Zoology, 64(1), 4956.

Storey, K. B., \& Storey, J. M. (1988). Freeze tolerance in animals. Physiological Reviews, 68(1), 27-84.

Storey, K. B. (1990). Life in a frozen state: adaptive strategies for natural freeze tolerance in amphibians and reptiles. American Journal of Physiology-Regulatory, Integrative and Comparative Physiology, 258(3), R559-R568.

Storey, K. B., Bischof, J., \& Rubinsky, B. (1992). Cryomicroscopic analysis of freezing in liver of the freeze-tolerant wood frog. American Journal of PhysiologyRegulatory, Integrative and Comparative Physiology, 263(1), R185-R194.

Storey, J. M., \& Storey, K. B. (1996). $\beta$-Adrenergic, hormonal, and nervous influences on cryoprotectant synthesis by liver of the freeze-tolerant wood frog Rana sylvatica. Cryobiology, 33(1), 186-195.

Storey, K. B. (1997). Organic solutes in freezing tolerance. Comparative Biochemistry and Physiology Part A: Physiology, 117(3), 319-326.

Storey, K. B., \& Storey, J. M. (2004). Metabolic rate depression in animals: transcriptional and translational controls. Biological Reviews, 79(1), 207-233.

Storey, K. B., \& Storey, J. M. (2007). Tribute to PL Lutz: putting life on pause-molecular regulation of hypometabolism. Journal of Experimental Biology, 210(10), 17001714. 
Storey, K. B., \& Storey, J. M. (2013). Molecular biology of freezing tolerance. Comprehensive Physiology, 3(3), 1283-1308.

Storey, K. B. (2015). Regulation of hypometabolism: insights into epigenetic controls. Journal of Experimental Biology, 218(1), 150-159.

Storey, K. B., \& Storey, J. M. (2017). Molecular physiology of freeze tolerance in vertebrates. Physiological Reviews, 97(2), 623-665.

Sullivan, K. J., \& Storey, K. B. (2012). Environmental stress responsive expression of the gene li16 in Rana sylvatica, the freeze tolerant wood frog. Cryobiology, 64(3), 192-200.

Thomashow, M. F. (1999). Plant cold acclimation: freezing tolerance genes and regulatory mechanisms. Annual review of plant biology, 50(1), 571-599.

Winter, J., Jung, S., Keller, S., Gregory, R. I., \& Diederichs, S. (2009). Many roads to maturity: microRNA biogenesis pathways and their regulation. Nature cell biology, 11(3), 228-234.

Wu, C. W., Biggar, K. K., \& Storey, K. B. (2013). Dehydration mediated microRNA response in the African clawed frog Xenopus laevis. Gene, 529(2), 269-275.

Wu, C. W., Biggar, K. K., \& Storey, K. B. (2014). Expression profiling and structural characterization of microRNAs in adipose tissues of hibernating ground squirrels. Genomics, proteomics \& bioinformatics, 12(6), 284-291.

Wu, C. W., Biggar, K. K., Luu, B. E., Szereszewski, K. E., \& Storey, K. B. (2016). Analysis of microRNA expression during the torpor-arousal cycle of a mammalian hibernator, the 13-lined ground squirrel. Physiological genomics, 48(6), 388-396.

Wu, L., Fan, J., \& Belasco, J. G. (2006). MicroRNAs direct rapid deadenylation of mRNA. Proceedings of the National Academy of Sciences of the United States of America, 103(11), 4034-4039.

Wu, P., Nakano, S. I., \& Sugimoto, N. (2002). Temperature dependence of thermodynamic properties for DNA/DNA and RNA/DNA duplex formation. European Journal of Biochemistry, 269(12), 2821-2830.

Zachariassen, K. E., \& Kristiansen, E. (2000). Ice nucleation and antinucleation in nature. Cryobiology, 41(4), 257-279.

Zhang, J., \& Storey, K. B. (2013). Akt signaling and freezing survival in the wood frog, Rana sylvatica. Biochimica et Biophysica Acta (BBA)-General Subjects, 1830(10), 4828-4837. 
CHAPTER 2

General Materials and Methods 


\subsection{Animal experiments}

Male wood frogs were collected from spring meltwater ponds in Oxford Mills, Ontario, Canada, and transported to Carleton University on crushed ice. Frogs were washed in a tetracycline bath and acclimated at $5^{\circ} \mathrm{C}$ for two weeks in plastic containers lined with sphagnum moss. Active control frogs were sampled from this condition.

For the frozen condition, frogs were moved to $-3^{\circ} \mathrm{C}$ plastic containers lined with damp paper towel and an initial (45 min) cooling period was used to initiate freezing during which body temperature cooled to below $-0.5^{\circ} \mathrm{C}$. Incubator temperatures were then raised to $-2.5^{\circ} \mathrm{C}$ and at which point the $24 \mathrm{~h}$ freezing was timed. Frogs were sampled after $24 \mathrm{~h}$ of freezing as this was the time required to reach maximal ice content formation (Storey, 1990). Frozen frogs were randomly sampled from this condition. The remaining frogs were assigned to the recovery group and were then transferred to $5^{\circ} \mathrm{C}$. Thawed frogs were sampled after $8 \mathrm{~h}$ of thawing at this temperature. Frogs were sampled after 8 hours of thawing as this was when they displayed a consistent "recovery phenotype", with a return of heartbeat, breathing, and limb movement.

All control, $24 \mathrm{~h}$ frozen, and $8 \mathrm{~h}$ thawed frogs were euthanized by spinal pithing. Brain and liver were then rapidly excised, flash frozen in liquid nitrogen, and stored at $-80^{\circ} \mathrm{C}$ until use. Animal care protocols and experimentation were performed in accordance with the Carleton University Animal Care Committee and followed the guidelines set by the Canadian Council on Animal Care. 


\subsection{Total soluble protein extraction}

Total soluble protein extracts were prepared from liver and brain tissue of control, $24 \mathrm{~h}$ frozen, $8 \mathrm{~h}$ thawed wood frog conditions. Samples $(500 \mathrm{mg})$ of frozen tissue were crushed with a mortar and pestle in liquid $\mathrm{N}_{2}$ and then homogenized 1:2 w:v using a Polytron PT10 homogenizer (Kinematica) and chilled homogenization buffer (20 mM HEPES, $200 \mathrm{mM}$ NaCl, 0.1 mM EDTA, 10 mM NaF, 1 mM Na $3 V_{4}, 10$ mM $\beta$-glycerophosphate, $p H$ 7.4) with a few crystals of PMSF and $1 \mu \mathrm{L} / \mathrm{mL}$ of protease inhibitor (Cat\# PIC002; BioShop Canada Inc., Burlington, ON, Canada). Homogenates were centrifuged for $15 \mathrm{~min}$ at $10,000 \mathrm{~g}\left(4^{\circ} \mathrm{C}\right)$, and soluble protein-containing supernatants were collected.

Total protein concentration of each sample was quantified using the BioRad protein assay (Cat\# 5000002; BioRad Laboratories, Hercules, CA, USA) at $595 \mathrm{~nm}$ on an MR5000 microplate reader (Dynatech Laboratories, Chantilly, VA, USA). Protein concentrations were standardized to $10 \mu \mathrm{g} / \mu \mathrm{L}$ using homogenization buffer. Standardized samples were mixed 1:1 v/v with SDS buffer (100 mM Tris-HCl, 4\% w/v SDS, 20\% v/v glycerol, 0.2 w/v bromophenol, $10 \% \mathrm{v} / \mathrm{v} \beta$-mercaptoethanol, $\mathrm{pH} 6.8$ ), to a final concentration of 5 $\mu \mathrm{g} / \mu \mathrm{L}$. Samples were boiled for $10 \mathrm{~min}$ to denature and linearize all proteins and were then stored at $-40^{\circ} \mathrm{C}$ until use.

\subsection{Immunoblotting}

Sodium dodecyl sulfate polyacrylamide gel electrophoresis (SDS-PAGE) was run by loading equal amounts $(25-40 \mu \mathrm{g})$ of control, $24 \mathrm{~h}$ frozen, and $8 \mathrm{~h}$ thawed total protein into 6-15\% discontinuous SDS polyacrylamide gels. Depending on the size of the protein of interest, either $4 \mu \mathrm{L}$ of PiNK Plus pre-stained protein ladder (10.5-175 kDa; Cat\# PM005- 
0500K; FroggaBio, Toronto, ON, Canada) or $6 \mu \mathrm{L}$ of BLUeye pre-stained protein ladder (10 to $245 \mathrm{kDa}$; Cat\# PM007-0500; FroggaBio) was loaded into one lane of each gel for reference. Upper stacking gels consisted of 4\% acrylamide v/v, 1 M Tris buffer [pH 6.8], $0.1 \%$ SDS, $0.1 \%$ ammonium persulphate (APS), and $0.1 \% \mathrm{~N}, \mathrm{~N}, \mathrm{~N}^{\prime}, \mathrm{N}^{\prime}$-tetramethylethane1,2-diamine (TEMED). Resolving gels were comprised of 6-15\% acrylamide v/v, $1.5 \mathrm{M}$ Tris buffer [pH 8.8], $0.1 \%$ SDS, $0.1 \%$ APS, and 0.1\% TEMED. Loaded SDS-PAGE gels were then run at $180 \mathrm{~V}$ for $1-4 \mathrm{~h}$ at $4^{\circ} \mathrm{C}$ in running buffer $(25 \mathrm{mM}$ Tris-base, $190 \mathrm{mM}$ glycine, $0.1 \%$ w/v SDS, [pH 7.6]), using a BioRad Mini Protean III system (BioRad Laboratories, Hercules, CA, USA). Resolved protein gels were then transferred to $0.45 \mu \mathrm{m}$ pore polyvinylidene difluoride (PVDF) membranes at $160 \mathrm{~mA}$ for $1.5-16 \mathrm{~h}$ in transfer buffer ( $25 \mathrm{mM}$ Tris-base, $192 \mathrm{mM}$ glycine 10\% v/v methanol, [pH 8.5]), at $4{ }^{\circ} \mathrm{C}$.

Transferred membranes were air-dried for $15 \mathrm{~min}$, reactivated in methanol for $5 \mathrm{~min}$ and incubated in $2-10 \% \mathrm{v} / \mathrm{v}$ skim milk in TBST $(20 \mathrm{mM}$ Tris base $[\mathrm{pH} 7.6], 140 \mathrm{mM} \mathrm{NaCl}$, $0.05 \%$ v:v Tween-20, and $90 \% \mathrm{v}: \mathrm{v} d d \mathrm{H}_{2} \mathrm{O}$ ). Membranes were subsequently incubated in skim milk (2-10\% v/v in TBST) for 15-30 min or in 30,000-70,000 MW polyvinyl alcohol (PVA) $(1 \mathrm{mg} / \mathrm{mL}$ in TBST) for $30-90 \mathrm{sec}$ on a rocker at room temperature. Blocking membranes with milk or PVA was performed to limit non-specific primary and secondary antibody binding. Blocked membranes were washed $3 \times 5$ min in TBST and subsequently probed with the primary antibody of interest $\left(1: 1000 \mathrm{v} / \mathrm{v}\right.$ dilution in TBST) rocking at $4^{\circ} \mathrm{C}$ overnight. For supplier information on the primary antibodies used, refer to Appendix B. Various steps were taken to ensure that the antibodies used in this study cross-reacted with the correct wood frog protein, for more information refer to Appendix $\boldsymbol{C}$. Membranes were then washed $3 \times 5$ min with TBST and probed for $30 \mathrm{~min}$ at room temperature with 
horseradish peroxidase (HRP)-conjugated goat anti-rabbit secondary antibody (1:8000 v/v

dilution in TBST; Cat\# APA002P; BioShop). Membranes were then washed 3 x 5 min in TBST. For the complete list of optimized immunoblotting conditions for each target protein, refer to Appendix D.

Protein bands were visualized using chemiluminescence with $\mathrm{H}_{2} \mathrm{O}_{2}$ and Luminol, and imaged using the ChemiGenius BioImaging System (Syngene, Frederick, MD, USA). Membranes were then stained with Coomassie blue $(0.25 \% \mathrm{w} / \mathrm{v}$ Coomassie Brilliant Blue,

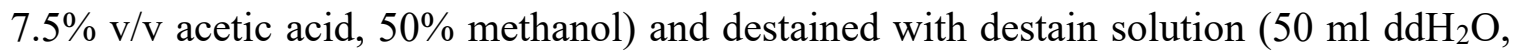
$50 \mathrm{ml}$ acetic acid, $150 \mathrm{ml}$ methanol) to visualize total protein as a loading control.

\subsection{RNA isolation}

For a general workflow of the microRNA experiments, data collection, and functional bioinformatics analyses refer to Figure 2.1. RNA isolation was performed on brain and liver tissues of control, $24 \mathrm{~h}$ frozen, $8 \mathrm{~h}$ thawed wood frogs ( $n=4$ individual animals). Samples (50-100 mg) of frozen tissue were crushed with a mortar and pestle in liquid $\mathrm{N}_{2}$ and then briefly homogenized in $1 \mathrm{~mL}$ of Trizol (Cat\# 15596-018; Invitrogen) using a Polytron PT1200 homogenizer (Kinematica). A $200 \mu \mathrm{L}$ aliquot of chloroform was then added and samples were centrifuged at $10,000 \mathrm{rpm}$ for $15 \mathrm{~min}$ at $4^{\circ} \mathrm{C}$. The upper aqueous phase was transferred to a new centrifuge tube and $500 \mu \mathrm{L}$ of isopropanol were added, mixing well. RNA was allowed to precipitate for $10 \mathrm{~min}$ on ice, then samples were centrifuged at 12,000 rpm for $15 \mathrm{~min}$ at room temperature. Pellets were washed with $1 \mathrm{~mL}$ of $70 \%$ ethanol and centrifuged at 7,500 rpm for $5 \mathrm{~min}$. Ethanol was decanted and RNA 
precipitates were allowed to air-dry for 10 min, followed by resuspending RNA in 30-50 $\mu \mathrm{L}$ of RNase-free water.

Concentration and purity of extracted RNA, as determined spectrophotometrically by the 260/280 $\mathrm{nm}$ ratio, was measured using a Take3 micro-volume quantification plate (BioTek) and a PowerWave HT spectrophotometer (BioTek). Only samples with 260/280 ratios greater than 1.8 were used for miRNA analyses. Next, total RNA integrity was examined using gel electrophoresis on a 1\% agarose gel stained with SYBR Green (Cat\# S7563; Invitrogen) and by verifying the presence of sharp bands for $28 \mathrm{~S}$ and $18 \mathrm{~S}$ ribosomal RNA. RNA isolates were then standardized to a final concentration of $1 \mu \mathrm{g} / \mu \mathrm{L}$ using RNase-free water and then frozen at $-80^{\circ} \mathrm{C}$ until further use. 


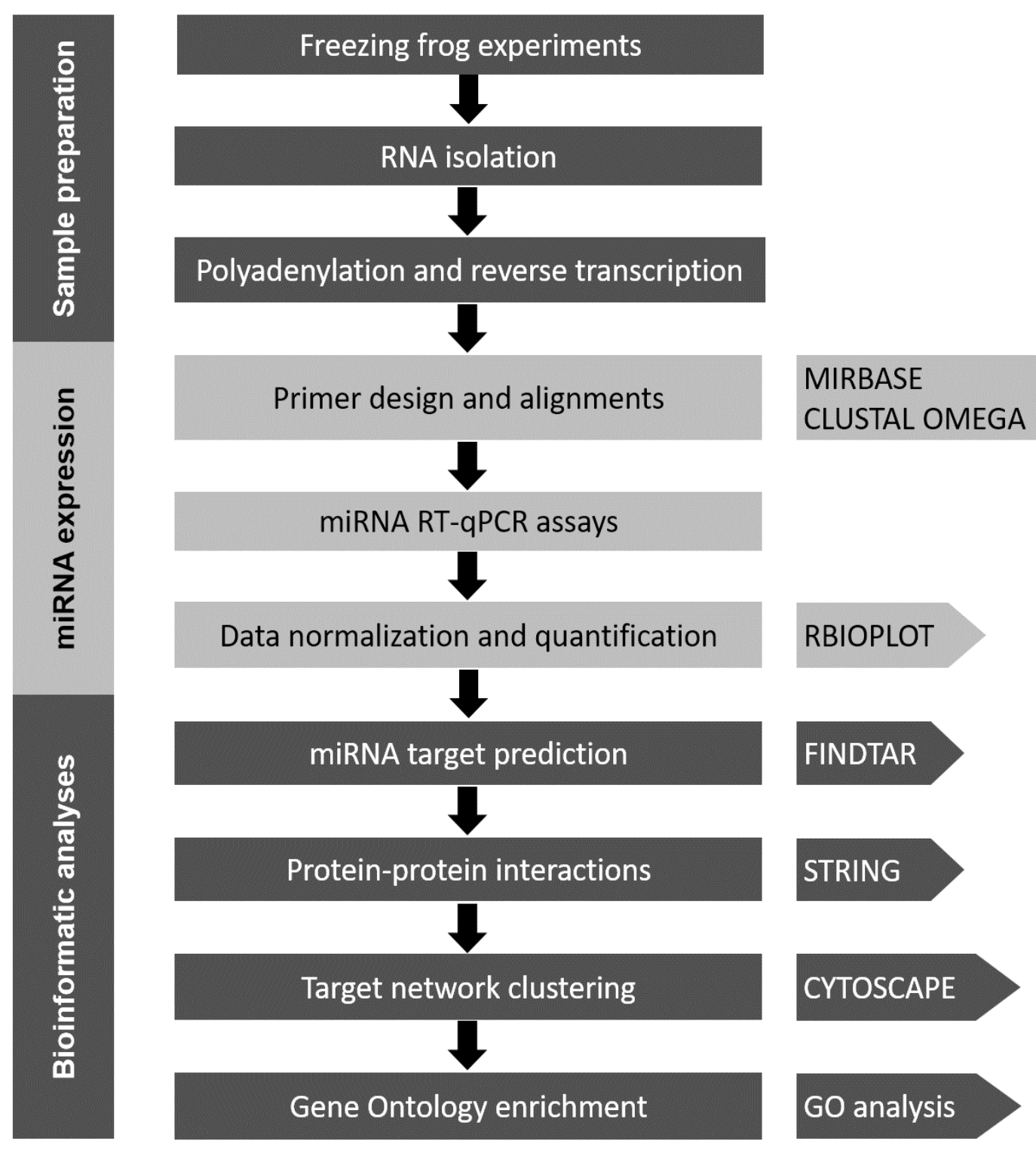

Figure 2.1. General workflow of microRNA experiments, data collection, and downstream functional bioinformatics analyses. Arrows on the right indicate the program and bioinfomtics tools used. 


\subsection{Polyadenylation and stem-loop reverse transcription}

Extracted RNA samples were prepared for miRNA analysis by polyadenylation, stem-loop adapter annealing, and reverse transcription (Biggar et al., 2014). Polyadenylation of miRNA was carried out using the PolyA tailing kit from Epi-Bio (Cat\# PAP5104H; Epicentre, Madison, WI, USA). Each $10 \mu \mathrm{L}$ reaction contained $3 \mu \mathrm{g}$ total RNA, $1 \mathrm{mM}$ ATP, and $0.5 \mu \mathrm{L}(2 \mathrm{U})$ of E. coli poly (A) polymerase in buffered solution ( $0.1 \mathrm{M}$ Tris$\mathrm{HCl}[\mathrm{pH} 8.0], 0.25 \mathrm{M} \mathrm{NaCl}$, and $10 \mathrm{mM} \mathrm{MgCl}_{2}$ ). Reactions were incubated at $37^{\circ} \mathrm{C}$ for 30 min to adenylate, $95^{\circ} \mathrm{C}$ for 5 min to arrest, and then immediately chilled on ice. For reverse transcription, the polyadenylated products (10 $\mu \mathrm{L}$ samples) were combined with $5 \mu \mathrm{L}$ of 250 pM stem-loop RT adapter primer (5'- CTC ACA GTA CGT TGG TAT CCT TGT GAT GTT CGA TGC CAT ATT GTA CTG TGA GTT TTT TTT TVN -3’'). The addition of degenerate nucleotides $\mathrm{N}$ (indicating nucleotides $\mathrm{A}, \mathrm{T}, \mathrm{C}$, or $\mathrm{G}$ ) and $\mathrm{V}$ (indicating nucleotides A, C, or G) to the 3' end of the stem-loop RT adapter primer ensures microRNA-specific sequence binding, despite the variable lengths of the polyadenylated tails (Biggar et al., 2014). This was then heated to $95^{\circ} \mathrm{C}$ for 5 min to denature RNA, cooled to $60^{\circ} \mathrm{C}$ for $5 \mathrm{~min}$ to allow annealing, and placed on ice for $1 \mathrm{~min}$. The SuperScript ${ }^{\mathrm{TM}}$ III Reverse Transcriptase kit (Cat\# 18080044; ThermoFisher Scientific) was used on the polyadenylated and stem-loop primer-annealed RNA samples. Briefly, each sample was combined with: $1 \mu \mathrm{L}$ mouse Maloney leukemia virus (M-MLV) reverse transcriptase (2 $\mathrm{U}), 1 \mu \mathrm{L}$ deoxynucleotide triphosphate (dNTP) mixture containing $25 \mathrm{mM}$ of each nucleotide (Cat\# R1121; ThermoFisher Scientific), $2 \mu \mathrm{L} 0.1 \mathrm{M}$ dithiothreitol (DTT), and 4 $\mu \mathrm{L} 5 \mathrm{x}$ first-strand buffer. Samples were incubated at $16^{\circ} \mathrm{C}$ for $30 \mathrm{~min}, 42^{\circ} \mathrm{C}$ for $30 \mathrm{~min}$, and $85^{\circ} \mathrm{C}$ for $5 \mathrm{~min}$. Products were serially diluted and frozen at $-20^{\circ} \mathrm{C}$. 

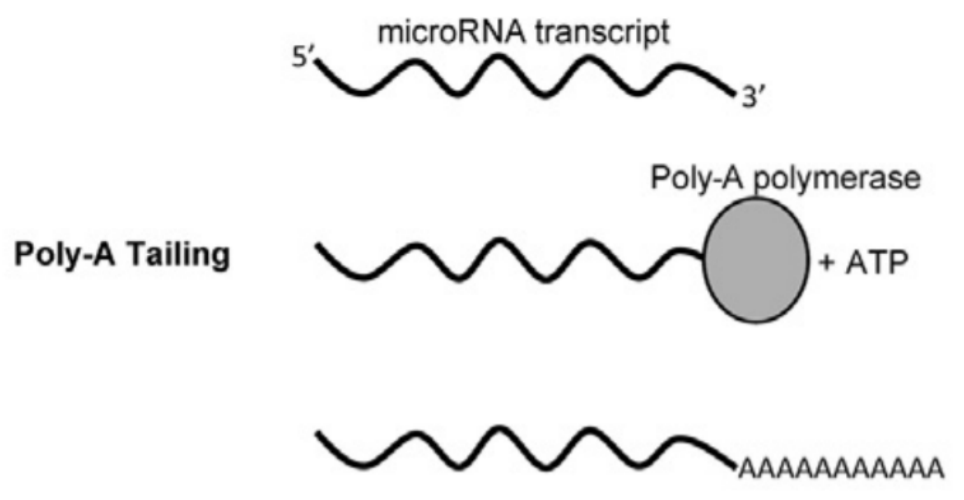

Adapter Ligation

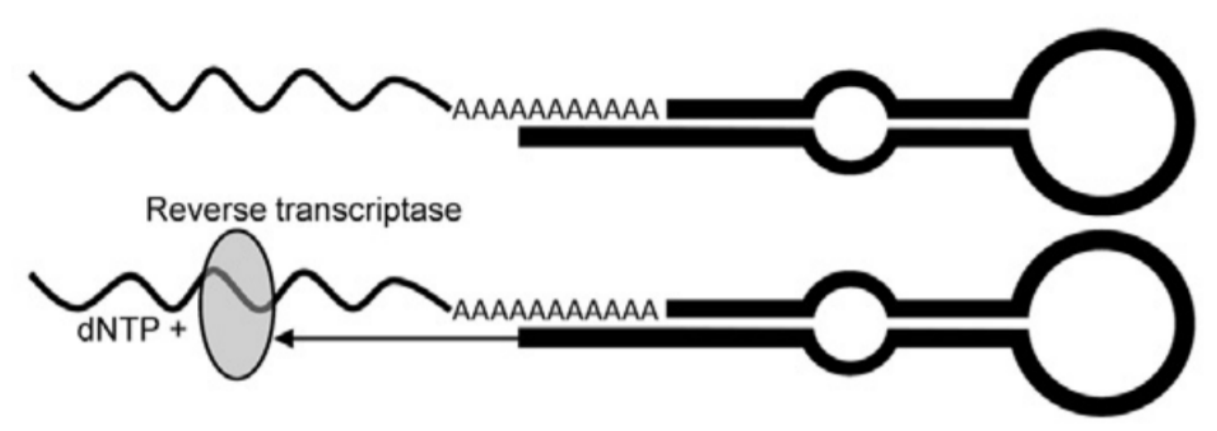

Figure 2.2. Schematic of the polyadenylated microRNA amplification protocol using stem-loop primers. A poly-A tail is added to microRNAs, followed by cDNA synthesis using an adapted primer and reverse transcriptase. The resulting cDNA is ready for RTqPCR amplification with a universal PCR primer and a microRNA-specific forward primer. This unique amplification system provides ease of use and efficient cDNA synthesis for the quantification of many mature microRNAs with a high degree of sensitivity and specificity. Figure from (Biggar et al., 2014). 


\subsection{MicroRNA sequence conservation and primer design}

The miRNA-specific forward primers were designed based on the annotated miRNA sequences from Xenopus tropicalis and Xenopus laevis genomes on XenBase (www.xenbase.org; accessed September 2016) and on the miRNA sequences deposited on miRBase (www.mirbase.org; Release 21). To validate the use of these primers for RTqPCR of miRNAs from $R$. sylvatica, the precursor miRNA stem-loop sequences of Xenopus were subjected to NCBI BLASTn (https://blast.ncbi.nlm.nih.gov/Blast.cgi) to assess miRNA conservation across taxa. Multiple sequence alignments were performed with EMBL-EBI CLUSTAL OMEGA (http://www.ebi.ac.uk/Tools/msa/clustalo/) with the stem-loop pre-miRNA sequences from Gallus gallus and Anolis carolinensis. In cases where sequences from these animals were unavailable or unannotated in NCBI GenBank, the alignments were conducted using sequences from various other vertebrates.

Each of the forward miRNA-specific primers were designed using the conserved 5' miRNA end, as to decrease the challenges associated with designing primers based on the conservation of miRNA between select evolutionarily related species. As such, all forward miRNA-specific primers were designed with a modified short adapter and the first 15 nucleotides from the 5' mature miRNA sequence (Biggar et al., 2014). Primers had the following general sequence 5'- ACA CTC CAG CTG GGN NNN NNN NNN NN -3', where nucleotides denoted with an $\mathrm{N}$ indicate miRNA-specific sequence binding regions that vary depending on the target miRNA. This sequence has been optimized for product size, primer pairing, melting temperatures, and any homo- or heterodimers that may occur. Refer to Appendix $\boldsymbol{E}$ for the individual primer sequences of all the miRNA species analyzed. Since all miRNA species have been polyadenylated, stem-loop adapter annealed, 
and reverse transcribed, this allowed for the use of a single universal reverse primer ( $5^{\prime}-$ CTC ACA GTA CGT TGG TAT CCT TGT G -3'). All primers were synthesized by Integrated DNA Technologies.

\subsection{RT-qPCR assays}

All RT-qPCR assays were performed as previously described (Pellissier et al., 2006) using a CFX Connect ${ }^{\mathrm{TM}}$ Real-Time PCR Detection System (Cat\# 1855201; BioRad), following MIQE guidelines (Bustin et al., 2009). Each $20 \mu \mathrm{L}$ RT-qPCR reaction consisted of $2 \mu \mathrm{L}$ of diluted RT product, $10.115 \mu \mathrm{L}$ of $\mathrm{ddH}_{2} \mathrm{O}, 4 \mu \mathrm{L}$ of $1 \mathrm{M}$ trehalose (Cat\# TRE222; BioShop), $2 \mu \mathrm{L}$ RT-qPCR buffer (100 mM Tris-HCl [pH 8.5], $500 \mathrm{mM} \mathrm{KCl,} \mathrm{1.5 \%} \mathrm{Triton} \mathrm{X-100,} 20$ $\mathrm{mM} \mathrm{MgCl} 2,2 \mathrm{mM}$ dNTPs, and $100 \mathrm{nM}$ fluorescein), $0.5 \mu \mathrm{L}$ formamide (Cat\# FOR001; BioShop), $0.5 \mu \mathrm{L}$ of $25 \mathrm{mM}$ miRNA-specific forward primer, $0.5 \mu \mathrm{L}$ of $25 \mathrm{mM}$ universal reverse primer, $0.1 \mu \mathrm{L}$ of 100x SYBR green mix diluted in dimethyl sulfoxide (DMSO) (Cat\# S7585; Invitrogen), $0.16 \mu \mathrm{L}$ of $25 \mathrm{mM}$ dNTPs, and $0.125 \mu \mathrm{L}$ of $5 \mathrm{U} / \mu \mathrm{L}$ Taq polymerase (Cat\# TAQ001.1; BioShop). Reactions were incubated in a 96-well plate at $95^{\circ} \mathrm{C}$ for $3 \mathrm{~min}$, followed by 40 cycles of $95^{\circ} \mathrm{C}$ for $15 \mathrm{~s}$ and $60^{\circ} \mathrm{C}$ for $1 \mathrm{~min}$. To ensure primer specificity and the amplification of a single PCR product only, all RT-qPCR assays were subjected to post-run melt-curve analysis; reactions that amplified multiple nonspecific products were rejected. 


\subsection{Bioinformatics microRNA target identification and enrichment}

To further characterize the potential miRNA targets of the differentially expressed miRNAs, mature miRNA sequences were searched against 3'UTR sequences from the $X$. tropicalis reference genome available on the UCSC table browsed (JGI 7.0/xenTro7) using the temperature sensitive miRNA target identification program FINDTAR3 (v.3.11.12) (http://bio.sz.tsinghua.edu.cn/) (Ye et al., 2008). When aligning small fragments of genetic sequences against a reference genetic sequence, mutations and mismatches, annotated as gaps, must be taken into consideration. As such, for the optimization of sequence alignment FINDTAR3 uses a scoring system to account for the number of gaps and the different gap sizes through the penalization of gap openings and gap extensions, respectively. FINDTAR determines the AT, GC, and GT weight scores with revised Gotoh's and Marks' methods (Ye et al., 2008). FINDTAR3 was used with the following parameters: AT and GC weight of 5 , GT weight of 2 , a gap opening penalty of -8 , a gap extension penalty of -2 , target duplex with maximum threshold free energy $-20 \mathrm{kcal} / \mathrm{mol}$, and demand strict 5 ' seed pairing. RNA secondary structure and target predictions were carried out at a temperature of $-2^{\circ} \mathrm{C}$ for miRNAs that significantly changed during freezing, and at $5^{\circ} \mathrm{C}$ for miRNAs that significantly changed during thawing. The FINDTAR3 parameters descried above were used to provide a "flexible" window for the selection of miRNA:mRNA seed region matching and target selection. For a detailed miRNA target prediction bioinformatics workflow, refer to Appendix $\boldsymbol{F}$.

The list of miRNA-targeted genes generated by FINDTAR3 were then functionally enriched to examine the potential interplay between miRNA-targeted mRNA. This was done by mapping protein-protein interactions using the STRING (http://string-db.org/) 
Xenopus tropicalis database (Szklarczyk et al., 2014). STRING is an integrated quality controlled database and web resource of known and predicted protein-protein interactions. These include direct (physical) and indirect (functional) interactions that stem from numerous sources including; genomic context predictions, high-throughput lab experiments, conserved co-expression, automated text mining, and existing knowledge in databases. The protein interaction network generated was subsequently modelled using CYTOSCAPE, a network analysis and visualization platform (http://www.cytoscape.org), (Shannon et al., 2003) and STRING combined scores were clustered using Markov clustering (MCL). MCL is a divisive clustering algorithm used for network partitioning and is commonly applied to complex biological networks and protein-protein interaction networks. MCL clustering was performed on the CYTOSCAPE ClusterMaker application. Pathway enrichment analysis for miRNA-targeted clusters was performed on miRNAs found to significantly change in response to freezing and thawing. This analysis was performed using the Gene Ontology (GO) Enrichment Analysis available for Xenopus tropicalis using the PANTHER classification system (v.11.1) (http://www.geneontology.org/). GO Enrichment Analysis was based on functional biological processes, wherein a biological process is a recognized as a series of molecular functions and events with a defined beginning and end.

\subsection{Quantification and Statistics}

Chemiluminescent immunoblot protein bands were quantified by densitometry using a ChemiGenius BioImaging System and GeneTools Software (Syngene, Frederick, MD, USA). Immunoblot band intensity in each lane was standardized against a group of 
Coomassie blue-stained protein bands to correct for any minor variations in sample loading. Immunoblot data for each experimental condition are expressed as means $\pm \mathrm{SEM}$ with $n=4$ samples from different animals. Statistical analysis was performed by one-way ANOVA and a Dunnett's post-hoc test $(p<0.05$ accepted as significant) using the RBIOPLOT statistics and graphing R package (Zhang and Storey, 2016).

For RT-qPCR, the comparative $\Delta \Delta \mathrm{Cq}$ method was used to calculate relative levels of miRNA expression (Bustin et al., 2009). Raw Cq values were transformed to the $2^{-\mathrm{Cq}}$ form, such that the miRNA of interest could be standardized to the endogenous controls, snord 68 (brain) and U6 snRNA (liver). Both sno68 and U6 snRNA were experimentally determined to be suitable reference genes based on their stable expression in $R$. sylvatica brain and liver, respectively, under all experimental conditions and using the calculations previously described (Schmittgen and Livak, 2008). Other small RNAs (non-miRNAs) were used as reference genes to control for the miRNA sample preparation protocol as these RNA species are also small and not polyadenylated. Data were collected and analyzed as mean relative expressions (mean $\pm \mathrm{SEM}$ ), where $n=4$ independent biological replicates from different animals at each sampling point with two highly comparable technical replicates for each quantification. Differences in miRNA relative expression between control, frozen, and thawed conditions were considered significant (indicated by *) when a one-way ANOVAs and Dunnett's post-hoc test yielded a $p<0.05$. Statistical analyses were performed using the RBIOPLOT statistics and graphing R package (Zhang and Storey, 2016). 


\section{REFERENCES [CHAPTER 2]}

Biggar, K. K., Wu, C. W., \& Storey, K. B. (2014). High-throughput amplification of mature microRNAs in uncharacterized animal models using polyadenylated RNA and stem-loop reverse transcription polymerase chain reaction. Analytical biochemistry, 462, 32-34.

Bustin, S. A., Benes, V., Garson, J. A., Hellemans, J., Huggett, J., Kubista, M., ... \& Vandesompele, J. (2009). The MIQE guidelines: minimum information for publication of quantitative real-time PCR experiments. Clinical chemistry, 55(4), 611-622.

Pellissier, F., Glogowski, C. M., Heinemann, S. F., Ballivet, M., \& Ossipow, V. (2006). Lab assembly of a low-cost, robust SYBR green buffer system for quantitative real-time polymerase chain reaction. Analytical biochemistry, 350(2), 310-312.

Reczko, M., Maragkakis, M., Alexiou, P., Grosse, I. \& Hatzigeorgiou, A. G. (2012). Functional microRNA targets in protein coding sequences. Bioinformatics, 28, 771-776.

Schmittgen, T. D., \& Livak, K. J. (2008). Analyzing real-time PCR data by the comparative CT method. Nature protocols, 3(6), 1101-1108.

Shannon, P., Markiel, A., Ozier, O., Baliga, N. S., Wang, J. T., Ramage, D., ... \& Ideker, T. (2003). Cytoscape: a software environment for integrated models of biomolecular interaction networks. Genome research, 13(11), 2498-2504.

Storey, K. B. (1990). Life in a frozen state: adaptive strategies for natural freeze tolerance in amphibians and reptiles. American Journal of Physiology-Regulatory, Integrative and Comparative Physiology, 258(3), R559-R568.

Szklarczyk, D., Franceschini, A., Wyder, S., Forslund, K., Heller, D., Huerta-Cepas, J., ... \& Kuhn, M. (2014). STRING v10: protein-protein interaction networks, integrated over the tree of life. Nucleic acids research, gku1003.

Vlachos, I. S. et al. (2012). DIANA miRPath v.2.0: investigating the combinatorial effect of microRNAs in pathways. Nucleic Acids Res. 40, W498-504.

Ye, W., Lv, Q., Wong, C. K. A., Hu, S., Fu, C., Hua, Z., ... \& Zhang, Y. (2008). The effect of central loops in miRNA: MRE duplexes on the efficiency of miRNAmediated gene regulation. PloS one, 3(3), e1719.

Zhang, J., \& Storey, K. B. (2016). RBioplot: an easy-to-use R pipeline for automated statistical analysis and data visualization in molecular biology and biochemistry. PeerJ, 4, e2436. 


\section{CHAPTER 3}

Frozen Brain MicroRNAs 


\section{Micromanaging freeze tolerance: The biogenesis and regulation of neuroprotective microRNAs in frozen brains}

Hanane Hadj-Moussa and Kenneth B. Storey*

Department of Biology and Institute of Biochemistry, Carleton University, 1125 Colonel By Drive, Ottawa, Ontario K1S 5B6, Canada

*Corresponding author: kenneth.storey@carleton.ca 


\section{ABSTRACT}

When temperatures plummet below $0^{\circ} \mathrm{C}$, the wood frog (Rana sylvatica) freezes up to $65 \%$ of its body water in extracellular ice masses, displaying no measurable brain activity, no breathing, no movement, and a flat-lined heart. To survive, freeze tolerant frogs retreat into a state of suspended animation characterized by global suppression of metabolic function and reprioritization of energy usage to essential survival processes that is elicited, in part by the regulatory controls of microRNAs. The present study is the first to investigate miRNA biogenesis and regulation in the brain of a freeze tolerant vertebrate. Indeed, proper brain function and adaptations to environmental stimuli play a critical role in coordinating stress responses. Immunoblotting of the miRNA biogenesis factors illustrated an overall reduction in the majority of processing proteins suggesting a potential suppression of miRNA maturation over the freeze-thaw cycle. This was coupled with a large-scale RTqPCR analysis of relative expression levels of 113 microRNA species in the brains of control, $24 \mathrm{~h}$ frozen, and $8 \mathrm{~h}$ thawed $R$. sylvatica. Of the 41 microRNAs differentially regulated during freezing and thawing, only two were significantly upregulated. Bioinformatic target enrichment of the downregulated miRNAs, performed at the low temperatures experienced during freezing and thawing, predicted their involvement in the potential activation of various neuroprotective processes such as synaptic signalling, intracellular signal transduction, and anoxia/ischemia injury protection. The predominantly downregulated microRNA fingerprint identified herein suggests a microRNA-mediated cryoprotective mechanism responsible for maintaining neuronal functions and facilitating successful whole brain freezing and thawing.

KEYWORDS: Rana sylvatica; Cryoprotection; miRNA; FINDTAR3; STRING 


\subsection{INTRODUCTION}

Freezing is deadly for most organisms. Yet despite the challenges it poses, freeze tolerance has arisen multiple times across the eukaryotic kingdom ranging from microorganisms, plants, insects, invertebrates, reptiles, and amphibians (Costanzo et al., 2008; Denlinger and Lee, 2010; Holmstrup, 2014; Storey and Storey, 2017; Strimbeck et al., 2015). One of the most well-studied and extreme vertebrate models of natural freeze tolerance is the wood frog, Rana sylvatica (also known as Lithobates sylvaticus), capable of freezing up to $65 \%$ of total body water in extracellular ice masses. When the wood frog retreats into its frozen state of suspended animation, that can last months at a time, it exhibits no measurable brain activity, no breathing, no movement, and a flat-lined heart (Storey, 1990). To freeze and thaw months later, unscathed, wood frogs have developed various key adaptations that include; [1] initiation of freezing above the freezing point of body fluids at $\left(-0.5^{\circ} \mathrm{C}\right)$, to minimize damaging instantaneous ice surges that occur if animals are extensively supercooled prior to freezing, [2] synthesis and distribution of cryoprotectant glucose to minimize cell volume reduction and limit extracellular ice formation, and [3] global metabolic rate depression coupled with the activation of select "survival" pathways that protect and stabilize cellular macromolecules (Storey and Storey, 2017).

Wood frogs are subject to numerous freeze-associated challenges such as; anoxia/ischemia, dehydration, hyperglycemia, osmotic shock, mechanical damage from ice crystallization, and even thawing brings with it the dangers of rapid oxygen reperfusion (Storey and Storey, 2017). To survive such extreme environmental stress, these frogs have evolved various protective mechanisms, such as the upregulation of protective antioxidant defenses (Dawson et al., 2015), coupled with the global reprioritization of energy consumption 
(Storey and Storey, 2004). While freezing itself is a prolonged dormant state that is characterized by global metabolic rate depression, entrance into the frozen state does not involve extensive gene expression changes (Storey, 2006). This is likely because the energy-limited frozen state does not facilitate major reorganizations of the cellular environment. To survive, it appears that the frog has adapted molecular mechanisms that are; [1] modulated by environmental stimuli, [2] applicable to nearly all biological pathways, [3] easily inducible, [4] not energetically costly, and [5] rapidly reversible to allow for smooth transitions between active and dormant states (Storey, 2015). Indeed, studies on various hypometabolic animals have found this complex hypometabolic state to be orchestrated by a multitude of molecular mechanisms that fit these criteria including; epigenetic modifications of DNA (e.g. methylation) and histones, transcription factors, changes in transcription factor activity and signal transduction, in addition to posttranscriptional regulation of mRNA transcripts by RNA-binding proteins and the inhibitory action of microRNAs (miRNAs) (Storey, 2015).

MicroRNAs are short ( $\sim 22 \mathrm{nt})$ non-coding RNA transcripts that have been predicted to target more than $60 \%$ of protein-coding genes in humans (Bartel, 2009; Ebert and Sharp, 2012). This large and highly-conserved group are proving to be master regulators of virtually all cell processes with broad controls stretching into cell cycle, signal transduction, and energy metabolism pathways, among others (Biggar and Storey, 2011; Leung and Sharp, 2010). MicroRNAs rely on sequence-specificity to mediate posttranscriptional gene suppression via either translational inhibition or mRNA degradation, where a single miRNA can target multiple mRNAs and each mRNA can have multiple miRNA binding sites (Bartel, 2009). Add to this, that the thermodynamic nature of 
miRNA-mRNA interactions is sensitive to changes in the cellular environment, such as temperature (Wu et al., 2002). This leaves the miRNA-mRNA duplex vulnerable to large fluctuations in body temperature such as those experienced by ectothermic species, where drops in body temperature result in a decrease of the free energy required for the formation of the RNA duplex which in turn favours the binding of miRNA to mRNA (Biggar and Storey, 2014; Wu et al., 2002). Indeed, events such as freezing have been shown to result in significant changes in miRNA functionality and the individual mRNA molecules they target (Biggar and Storey, 2015).

The biogenesis and processing of microRNAs is under tight spatial and temporal control (Ha and Kim, 2014). Canonical microRNA biogenesis is a stepwise process that progresses from long imperfect dsRNA-like hairpins known as primary-miRNAs (pri-miRNAs). Maturation ensues with the cleavage of the 5' and 3' ends by DROSHA, a Class 2 RNase III endonuclease, and its binding partner DiGeorge's syndrome critical region gene 8 (DGCR8), a dsRNA binding protein, to release the $\sim 60-70 \mathrm{nt}$ precursor-miRNA (premiRNA) (Huang et al., 2011). Pre-miRNA then complexes with the nucleoplasmic transporter factor EXPORTIN-5 (XPO5) and RAN-GTP to protect against nuclear degradation and facilitate nuclear export into the cytoplasm for further processing (Bohnsack et al., 2004). Once in the cytoplasm, the RNAse III endonuclease DICER cleaves the loop structures to yield $\sim 21 \mathrm{nt}$ miRNA duplexes (Jaskiewicz and Filipowicz, 2008; MacFarlane and Murphy, 2010). This is facilitated by transactivator response RNA binding protein (TRBP) and protein kinase RNA activator (PACT) that complex with DICER to modulate efficient pre-miRNA processing (Fukunaga et al., 2012; Lee and Doudna, 2012). While one of the miRNA strands is usually degraded, the mature guide 
strand is incorporated into the effector (RISC) complex along with a member of the Argonaute family of endonucleases (AGO1-4), where miRNAs then use base-pairing to negatively regulate the expression of target mRNAs (Bartel, 2009).

Proper brain development and function rely on the gene-regulatory capacity of miRNA networks to coordinate between the transcriptomes and proteomes of the diverse neural cell types and their functional specializations (Abe and Bonini, 2013; O'Carroll and Schaefer, 2013). The brain's persistent flow of information and adaptations to environmental stimuli plays a pivotal role in the facilitation of stress responses and prolonged hypometabolic periods (Storey and Storey, 2007). Despite the lack of any measurable brain activity or nerve conductance (Storey and Storey, 2017), biochemical studies on the brains of frozen $R$. sylvatica have revealed various neuroprotective aspects such as the increase in novel lil6 and frlo which have been linked with the mitigation of oxygen restriction damages and freeze protection, increased levels of c-FOS, and the elevation of phosphorylated Smad3 levels that has been implicated in the promotion of tissue survival (Aguilar et al., 2016; Cai and Storey, 1997; Greenway and Storey, 2000; Sullivan and Storey, 2012). Other neuronal "activations" found to occur in frozen wood frog brains include; the upregulation of protein kinase $\mathrm{C}$ phosphorylation status, the upregulation of the acidic ribosomal phosphoprotein $\mathrm{P} 0$, and increased levels of the large ribosomal subunit protein 7 (Dieni and Storey, 2014; Wu et al., 2008; Wu and Storey, 2005). Our initial investigations into the regulatory response of miRNAs in wood frog heart, skeletal muscle, and liver have demonstrated tissue-specific differential expression of miRNAs involved in the maintenance of muscle contraction and reversible protein phosphorylation over the freeze-thaw cycle (Bansal et al., 2016; Biggar et al., 2009). 
A growing number of studies by our lab, and others, have implicated miRNAs in various stress responses and adaptations in diverse animals ranging from; marsupial hibernation (Hadj-Moussa et al., 2016), sea cucumber estivation (Chen and Storey, 2014), invertebrate anoxia tolerance (Biggar et al., 2012), insect freeze-avoidance (Lyons et al., 2015), and turtle, frog, and insect freeze tolerance (Lyons et al., 2013; Storey and Storey, 2017). In the present study, which to our knowledge is the first investigation of miRNAs in freeze tolerant brains, immunoblotting of the miRNA biogenesis proteins revealed a potential suppression of miRNA processing over the freeze-thaw cycle in wood frog brains. Largescale analysis of the expression levels of 113 miRNAs, using RT-qPCR, found that the 41 differentially regulated miRNAs displayed a pattern of downregulation during both freezing and thawing. Bioinformatic miRNA target prediction of the downregulated miRNAs was then performed at physiologically relevant low temperatures of $-2^{\circ} \mathrm{C}$ and $5^{\circ} \mathrm{C}$, for $24 \mathrm{~h}$ frozen and $8 \mathrm{~h}$ thawed conditions, respectively. Functional enrichment and clustering revealed that the mRNA targets of the downregulated miRNAs networks mediate neuroprotective processes and hypoxia/anoxia protection. Our findings suggest a miRNA-mediated mechanism for tuning of neuronal functions and brain cryoprotection over the course of the freeze-thaw cycle. Understanding the role that miRNAs play in facilitating successful freeze tolerance and protecting living brain tissue and neuronal networks from freeze-associated damages sheds light on new approaches on the basic processes that must be maintained for successful brain function. 


\subsection{MATERIALS AND METHODS}

\subsubsection{Animals}

Rana sylvatica mature males were collected from spring meltwater ponds in the Ottawa region and transported on ice to an animal facility at Carleton University. Frogs were washed in a tetracycline bath and acclimated at $5^{\circ} \mathrm{C}$ for two weeks in containers lined with sphagnum moss. Three experimental treatments were run in parallel with individuals randomly selected for control, frozen and thawed conditions. Active (non-stressed) control frogs were sampled at this condition. For freezing experiments, frogs were placed in an incubator at $-3^{\circ} \mathrm{C}$ in plastic containers lined with damp paper towels. An initial (45 min) cooling period was used to initiate freezing during which the body temperature cooled to below $-0.5^{\circ} \mathrm{C}$, and at which point ice nucleation was triggered due to skin contact with ice crystals formed on the paper towels, as previously described (Aguilar et al., 2017). Following the initial $45 \mathrm{~min}$, the incubator temperature was raised to $-2.5^{\circ} \mathrm{C}$ and the length of maximal ice content formation ( $24 \mathrm{~h}$ ) was timed from this point (Storey, 1990). Frogs were randomly assigned to either the freezing group, that were then sampled after the $24 \mathrm{~h}$ freezing exposure, or assigned to the recovery group and were subsequently transferred to $5^{\circ} \mathrm{C}$ and sampled after $8 \mathrm{~h}$ of thawing. Animals were euthanized by spinal pithing and brains were rapidly dissected, immediately flash frozen in liquid nitrogen, and then subsequently stored at $-80^{\circ} \mathrm{C}$ until use. Animal care protocols, experimentation, and euthanasia were performed in accordance with the Carleton University Animal Care Committee and followed the guidelines set forth by the Canadian Council on Animal Care. 


\subsubsection{Total protein extraction}

Total protein extracts were prepared as previously described (Aguilar et al., 2016). Briefly, $\sim 500 \mathrm{mg}$ of brain tissue ( $n=4$ individual animals) were homogenized in 1:2 w:v chilled homogenization buffer (20 mM Hepes, $200 \mathrm{mM} \mathrm{NaCl}, 0.1 \mathrm{mM}$ EDTA, $10 \mathrm{mM} \mathrm{NaF}, 1 \mathrm{mM}$ $\mathrm{Na}_{3} \mathrm{VO}_{4}, 10 \mathrm{mM} \beta$-glycerophosphate, $\mathrm{pH}$ 7.5) with a few crystals of phenylmethylsulfonyl fluoride (Bioshop) and $1 \mu \mathrm{L} / \mathrm{mL}$ of protease inhibitor (Cat\# PIC002; Bioshop Canada Inc., Burlington, ON, Canada) using a Polytron PT10 homogenizer. Samples were then centrifuged at $4^{\circ} \mathrm{C}$ for $15 \mathrm{~min}$ at $10,000 \mathrm{~g}$, and soluble protein-containing supernatants were collected. Protein concentrations were measured using the BioRad reagent (Cat\# 5000002; Hercules, CA) as per the manufacturer's instructions. Soluble brain protein concentrations were standardized to $10 \mu \mathrm{g} / \mu \mathrm{L}$ using homogenization buffer and mixed with 1:1 v:v SDS buffer (100 mM Tris-HCl, 4\% w:v SDS, 20\% glycerol, 0.2\% w:v bromophenol blue, $10 \%$ $\mathrm{v}: \mathrm{v} \beta$-mercaptoethanol, $\mathrm{pH} 6.8$ ) to yield a final concentration of $5 \mu \mathrm{g} / \mu \mathrm{L}$. Samples were then boiled for $10 \mathrm{~min}$ and subsequently stored at $-20^{\circ} \mathrm{C}$ until use.

\subsubsection{Immunoblotting}

Equal amounts of total protein homogenates (20-40 $\mu \mathrm{g}$ depending on target being probed) from each sample were loaded onto $6-15 \%$ polyacrylamide gels and prepared with $5 \%$ upper stacking gels. Proteins were then separated in SDS-PAGE running buffer (190 mM glycine, $0.1 \% \mathrm{w} / \mathrm{v}$ SDS, $25 \mathrm{mM}$ Tris-base $[\mathrm{pH} 6.8]$ ) at $180 \mathrm{~V}$ for $60-120 \mathrm{~min}$ at $4^{\circ} \mathrm{C}$ with a

BioRad Mini Protean III system. Proteins were then electroblotted by wet transfer onto a $0.45 \mu \mathrm{m}$ polyvinylidene difluoride membrane in a pre-chilled transfer solution $(25 \mathrm{mM}$ Tris [pH 8.8], $192 \mathrm{mM}$ glycine, and $10 \% \mathrm{v} / \mathrm{v}$ methanol) at $4^{\circ} \mathrm{C}$ for $1-16 \mathrm{~h}$ at $160 \mathrm{~mA}$. Transferred membranes were air-dried for $15 \mathrm{~min}$, reactivated in methanol for $5 \mathrm{~min}$ and 
incubated in $2-10 \% \mathrm{v} / \mathrm{v}$ skim milk in TBST $(20 \mathrm{mM}$ Tris base $[\mathrm{pH} 7.6], 140 \mathrm{mM} \mathrm{NaCl}$, $0.05 \% \mathrm{v}: \mathrm{v}$ Tween-20, and $90 \% \mathrm{v}: \mathrm{v}$ dd $\mathrm{H}_{2} \mathrm{O}$ ) and blocked for $10-45 \mathrm{~min}$ in 2-10\% w:v milk in TBST. This was followed by $3 \times 5$ min washes with TBST and overnight incubations with the appropriate primary antibodies (1:1000 v:v dilution in TBST) at $4^{\circ} \mathrm{C}$. Primary antibodies were purchased for DROSHA (NeoBiolab; Cat\# A8336), DGCR8 (GeneTex; Cat\# GTX130061), DICER (SantaCruz; Cat\# SC-30226), AGO1 (GeneTex; Cat\# GTX47799), AGO2 (ECM BioSciences; Cat\# AP5281), p-AGO2 ${ }^{\text {Tyr393 }}$ (ECM BioSciences; Cat\# AP5311), RAN (GeneTex; Cat\# GTX114139), EXPORTIN-5 (GeneTex; Cat\# 130727), TRBP (GeneTex; Cat\# GTX485546), and PACT (GeneTex; Cat\# GTX114215). After probing with primary antibody, membranes were washed for $3 \times 5$ min with TBST and incubated with HRP-linked anti-rabbit secondary antibody (BioShop; 1:8000 v:v dilution in TBST) for $30 \mathrm{~min}$ at room temperature. Membranes were then washed in TBST and bands were visualized with enhanced chemiluminescence $\left(\mathrm{H}_{2} \mathrm{O}_{2}\right.$ and Luminol) on a ChemiGenius Bio-Imaging System (Syngene, Frederick, MD). Membranes were then stained with Coomassie blue $(0.25 \% \mathrm{w} / \mathrm{v}$ Coomassie brilliant blue, $7.5 \%$ v:v acetic acid, and $50 \%$ v:v methanol) to visualize total protein levels.

\subsubsection{Total RNA extraction}

Isolation of RNA was conducted as previously described (Hadj-Moussa et al., 2016). Approximately $50 \mathrm{mg}$ of brain tissue ( $\mathrm{n}=4$ individual animals) were briefly homogenized using a Polytron PT1200 homogenizer in $1 \mathrm{~mL}$ of Trizol (Invitrogen; Cat\# 15596-018), as per the manufacturers instructions. RNA quality was assessed by measuring the 260/280 nm ratio (>1.8) using a Take3 micro-volume quantification plate (BioTek) and a PowerWave HT spectrophotometer (BioTek). Total RNA integrity was determined by 
running RNA isolates on a 1\% agarose gel stained with SYBR Green and verifying the presence of sharp bands for $28 \mathrm{~S}$ and $18 \mathrm{~S}$ ribosomal RNA. All RNA samples were standardized to a final concentration of $1 \mu \mathrm{g} / \mu \mathrm{L}$ with RNase-free water. Samples were frozen at $-80^{\circ} \mathrm{C}$ until use.

\subsubsection{MicroRNA polyadenylation and stem-loop reverse transcription}

RNA samples were prepared for miRNA analysis as previously described (Biggar et al., 2014). Polyadenylation was carried out using the PolyA tailing kit from Epi-Bio (Cat\# PAP5104H; Epicentre, Madison, WI, USA). Each $10 \mu \mathrm{L}$ reaction contained; $3 \mu \mathrm{g}$ RNA, 1 $\mathrm{mM}$ ATP, and $0.5 \mu \mathrm{L}(2 \mathrm{U})$ of $E$. coli poly (A) polymerase in buffered solution $(0.1 \mathrm{M}$ Tris- $\mathrm{HCl}$ [pH 8.0], $0.25 \mathrm{M} \mathrm{NaCl}$, and $10 \mathrm{mM} \mathrm{MgCl}_{2}$ ). Reactions were incubated for $30 \mathrm{~min}$ at $37^{\circ} \mathrm{C}$ to adenylate, for $5 \mathrm{~min}$ at $95^{\circ} \mathrm{C}$ to arrest, and immediately chilled on ice. Stemloop adapter ligation was carried out by combining the polyadenylated products $(10 \mu \mathrm{L}$ samples) with $5 \mu \mathrm{L}$ of $250 \mathrm{pM}$ stem-loop adapter primers (Supp. Table S3.1) and heating the mixture for $5 \mathrm{~min}$ at $95^{\circ} \mathrm{C}$ to denature RNA, cooling it for $5 \mathrm{~min}$ at $60^{\circ} \mathrm{C}$ to allow annealing, and then immediately chilling on ice. For reverse transcription, each polyadenylated and stem-loop ligated RNA sample $(15 \mu \mathrm{L})$ was then combined with: $1 \mu \mathrm{L}$ mouse Maloney leukemia virus (M-MLV) reverse transcriptase (2 U) (Cat\# 18080044; ThermoFisher Scientific), $1 \mu \mathrm{L}$ deoxynucleotide triphosphate (dNTP) mixture containing $25 \mathrm{mM}$ of each nucleotide (Cat\# R1121; ThermoFisher Scientific), $2 \mu \mathrm{L} 0.1 \mathrm{M}$ dithiothreitol (DTT), and $4 \mu \mathrm{L}$ 5x first-strand buffer (Cat\# 18080044; ThermoFisher Scientific). Samples were then incubated at $16^{\circ} \mathrm{C}$ for $30 \mathrm{~min}, 42^{\circ} \mathrm{C}$ for $30 \mathrm{~min}$, and $85^{\circ} \mathrm{C}$ for 5 min. Polyadenylated, stem-loop ligated, and reverse transcribed cDNA was serially diluted and stored at $-20^{\circ} \mathrm{C}$. 


\subsubsection{Relative microRNA quantification}

All RT-qPCR assays were performed using a BioRad MyIQ2 Detection System (BioRad, Hercules, CA, USA), as previously described (Hadj-Moussa et al., 2016). The miRNAspecific forward primers were designed based on annotated miRNA sequences of Xenopus obtained from miRBase (Release 21). All miRNA-specific forward primers and the universal reverse primer were designed using the previously described method to amplify conserved miRNAs (Biggar et al., 2014) (Supp. Table S3.1). Primers were synthesized by Integrated DNA Technologies. To ensure primer specificity and the amplification of a single PCR product, all RT-qPCR assays were subjected to a post-run melt-curve analysis; reactions that amplified non-specific products were rejected.

\subsubsection{Bioinformatic microRNA target identification and pathway enrichment}

To characterize the potential miRNA targets, mature miRNA sequences were searched against the 3'UTR sequences from the $X$. tropicalis reference genome available on the UCSC table browser (JGI 7.0/xenTro7) using FINDTAR3 (v.3.11.12) (Ye et al., 2008). FINDTAR3 was used with the following parameters: AT and GC weight of 5, GT weight of 2, a gap opening penalty of -8 , a gap extension penalty of -2 , target duplex with maximum threshold free energy $-20 \mathrm{kcal} / \mathrm{mol}$, and demand strict 5' seed pairing. Target predictions were carried out at a temperature of $-2^{\circ} \mathrm{C}$ for miRNAs differentially expressed during freezing and at $5^{\circ} \mathrm{C}$ for miRNAs that changed during thawing. FINDTAR3 generated a list of miRNA-targeted genes that were then functionally enriched and analyzed. To characterize the functions and potential interplay between miRNA-targeted mRNA, protein-protein interactions were mapped using only the high-confidence STRING Xenopus tropicalis interaction database (Szklarczyk et al., 2015). This was subsequently 
modelled using CYTOSCAPE software (Shannon et al., 2003) and clustered using Markov clustering (MCL) default clustering parameters on the STRING combined scores. Two groups were queried separately: [1] the 23 miRNAs downregulated in frozen brains, and [2] the 33 miRNAs downregulated in thawed brains. Pathway enrichment analysis for miRNA-targeted clusters was performed on miRNAs found to significantly changing in response to freezing and thawing. This analysis was performed using the Gene Ontology (GO) Enrichment Analysis available for X. tropicalis using the PANTHER classification system (v.11.1). The enriched biological processes discussed herein are statistically significant clusters analyzed with Bonferroni tests and with $p<0.05$.

\subsubsection{Data quantification and statistics}

For relative protein level quantification, chemiluminescent bands were quantified by densitometry using a ChemiGenious Biolmaging Sytem and GeneTools Software (Syngene, MD, USA). To correct for minor variations in sample loading, the band intensity in each lane was standardized against a group of Coomassie blue-stained protein bands. Immunoblot data is expressed as means \pm SEM, relative to control values $(n=3-4$ from different animals). Data were statistically analyzed using one-way ANOVAs and a Dunnett's post-hoc test, results were considered significant when a Dunnett's test resulted in $p<0.05$. Statistical analyses were performed using the RBIOPLOT statistics and graphing R package (Zhang and Storey, 2016).

For RT-qPCR analyses, the comparative $\Delta \Delta \mathrm{Cq}$ method was used to calculate the relative miRNA expression levels. Raw $\mathrm{Cq}$ values were transformed to the $2^{-\mathrm{Cq}}$ form, each individual miRNA was then normalized to the reference gene, snord68. Snord68 RNA was experimentally deemed a suitable reference gene as it exhibited stable expression under 
control, frozen, and thawed conditions, as previously described (Schmittgen and Livak, $2008)$. Data is reported as mean expression levels, relative to control values ( $\pm \mathrm{SEM}$, where $n=3-4$ independent biological replicates of tissue from different animals). Data were statistically analyzed using a one-way ANOVA and a Dunnett's post-hoc test, miRNAs were considered significantly changing when $p<0.05$. Statistical analysis and histogram generation was performed using RBIOPLOT (Zhang and Storey, 2016).

\subsection{RESULTS:}

\subsubsection{Protein expression of miRNA biogenesis and processing machinery}

We analyzed protein abundance relative to the control of key proteins involved in the microRNA biogenesis pathway using immunoblotting in wood frog brains over a freezethaw cycle. Levels of DROSHA and DGCR8, the two sole members of the microprocessor complex, were found to significantly decrease during $24 \mathrm{~h}$ freezing to $0.666 \pm 0.042$ and $0.667 \pm 0.064$ of control levels, respectively (Fig. 3.1). This decrease was sustained throughout $8 \mathrm{~h}$ thawing with DROSHA and DGCR8 levels at $0.628 \pm 0.045$ and $0.432 \pm$ 0.012 of control, respectively (Fig. 3.1). EXPORTIN-5 protein levels significantly decreased in the brain during freezing and thawing to $0.749 \pm 0.041$ and $0.767 \pm 0.037$ of control, respectively (Fig. 3.1). DICER and RAN protein levels remained constant over the freeze-thaw cycle (Fig. 3.1). TRBP protein levels also remained constant during freezing but significantly decreased to $0.584 \pm 0.037$ of control during thawing (Fig. 3.1). Protein levels of the dsRNA binding protein PACT were found to decrease to $0.546 \pm 0.028$ of control during freezing and return to control levels during thawing (Fig. 3.1). AGO1 
protein levels were found to remain constant over the freeze-thaw cycle (Fig. 3.1). Total protein levels of the predominant AGO2 were found to significantly decrease during both freezing and thawing stress to $0.754 \pm 0.045$ and $0.556 \pm 0.012$ of control, respectively (Fig. 3.1). Levels of p-AGO2 $2^{\mathrm{Ty} 393}$ remained unchanged during freezing but significantly decreased to $0.602 \pm 0.03$ of control during $8 \mathrm{~h}$ thawing.

\subsubsection{Differential miRNA expression over the freeze-thaw cycle}

The primary goal of this study was to identify freeze and thaw-specific patterns of miRNA expression in the brains of $R$. sylvatica. Of the 113 miRNAs successfully quantified by RTqPCR, 41 miRNAs were differentially regulated over the freeze-thaw cycle, relative to control levels (Fig. 3.2). During freezing, 24 miRNAs were found to significantly change (Fig. 3.2). Nearly all of the miRNAs differentially expressed during freezing were found to be downregulated, with 23 miRNAs significantly decreasing to $0.421-0.845$ of control levels. The subset of miRNAs significantly downregulated during freezing comprised of; rsy-miR-103/107-3p, rsy-miR-10b-5p, rsy-miR-125a-5p, rsy-miR-126-3p, rsy-miR-140-5p, rsy-miR-145-5p, rsy-miR-155-5p, rsy-miR-183-5p, rsy-miR-184-3p, rsy-miR-18-5p, rsymiR-193-3p, rsy-miR-196b-5p, rsy-miR-204-5p, rsy-miR-210-3p, rsy-miR-214-3p, rsymiR-24a-5p, rsy-miR-2970-5p, rsy-miR-30b-5p, rsy-miR-30d-5p, rsy-miR-31b-5p, rsy$m i R-367-3 p$, $r s y-m i R-449 b-5 p$, and $r s y-m i R-9407-5 p$ (Fig. 3.2 and Supp. Table S3.2). Only $r s y-m i R-451-5 p$ expression levels significantly increased during freezing to $2.963 \pm$ 0.445 of control (Fig. 3.2 and Supp. Table S3.2).

During thawing, 34 miRNAs were found to be differentially expressed (Fig. 3.2). Similar to the pattern observed during freezing, the majority of the differentially expressed 
miRNAs were found to significantly decrease during thawing, with 33 miRNAs significantly decreasing to $0.421-0.861$ of control (Fig. 3.2 and Supp. Table S3.2). The subset of miRNAs downregulated during thawing are; rsy-miR-let-7f-3p, rsy-miR103/107-3p, rsy-miR-10b-5p, rsy-miR-125a-5p, rsy-miR-126-3p, rsy-miR-129-5p, rsy$m i R-130 a-5 p, r s y-m i R-140-5 p, r s y-m i R-145-5 p, r s y-m i R-155-5 p, r s y-m i R-181 a-3 p, r s y-$ miR-183-5p, rsy-miR-184-3p, rsy-miR-193-3p, rsy-miR-196b-5p, rsy-miR-204-5p, rsymiR-208-3p, rsy-miR-210-3p, rsy-miR-212-3p, rsy-miR-221-3p, rsy-miR-222-3p, rsy-miR22-5p, rsy-miR-24a-5p, rsy-miR-26-3p, rsy-miR-26-5p, rsy-miR-30b-5p, rsy-miR-30d-5p, $r s y-m i R-31 b-5 p, r s y-m i R-365-3 p, r s y-m i R-425-5 p, r s y-m i R-449 c-3 p, r s y-m i R-96-5 p$, and $r s y-m i R-98-5 p$ (Fig. 3.2 and Supp. Table S3.2). Despite a backdrop of unchanging and downregulated miRNAs, $r s y-m i R-192-5 p$ was upregulated during thawing to $2.56 \pm 0.457$ of control.

A subset of miRNAs were found to be downregulated during both freezing and thawing; rsy-miR-103/107-3p, rsy-miR-10b-5p, rsy-miR-125a-5p, rsy-miR-126-3p, rsy-miR-140-5p, $r s y-m i R-145-5 p, r s y-m i R-155-5 p, r s y-m i R-183-5 p, r s y-m i R-184-3 p, r s y-m i R-193-3 p, r s y-$ $m i R-196 b-5 p, \quad r s y-m i R-204-5 p, \quad r s y-m i R-210-3 p, \quad r s y-m i R-24 a-5 p, \quad r s y-m i R-30 b-5 p, \quad r s y-$ $m i R-30 d-5 p$, and $r s y-m i R-31 b-5 p$ (Fig. 3.2 and Supp. Table S3.2).

\subsubsection{Bioinformatic analyses of miRNA-targeted pathways}

Using miRNA-mRNA target predictions at physiologically relevant temperatures, proteinprotein interaction networks, and biological process enrichment, the following targeted gene networks and processes were identified. Clusters predicted that the key pathways targeted by the miRNAs downregulated during the $24 \mathrm{~h}$ frozen condition consisted of; [1] intracellular signal transduction pathways, [2] RNA processing and mRNA splicing, [3] 
synaptic signalling, [4] lipid phosphorylation, [5] microtubule nucleation, [6] DNA replication, [7] generation of precursor metabolites and energy, [8] developmental process, and [9] proton transport and ATP hydrolysis (Fig. 3.3 and Supp. Table S3.3). The key networks targeted by the miRNAs downregulated in the $8 \mathrm{~h}$ thawing condition were comprised of; [1] intracellular signal transduction, [2] DNA replication, [3] proton transport, [4] RNA processing and mRNA splicing, [5] protein folding and chaperones, [6] synaptic signalling, [7] ATP hydrolysis, [8] microtubule nucleation, [9] generation of

precursor metabolites and energy, [10] lipid phosphorylation, [11] oligosach-lipid intermediate biosynthesis, and [12] developmental process (Fig. 3.4 and Supp. Table S3.4). The majority of remaining clusters in both freezing and thawing remained unclassified, likely due to the limited nature of annotated X. tropicalis network interactions.

\subsection{DISCUSSION}

The rapid and reversible nature of miRNA-mediated gene silencing renders them excellent candidates for fine tuning the gene expression profile required to protect brain networks and processes during prolonged periods of severe environmental stress. Studies on diverse animal models have demonstrated that the brain is critical for coordinating environmental stimuli and for mounting a molecular stress responses. While global metabolic rate depression is one of the main characteristics of freeze tolerance, neuroprotective processes such as the promotion of tissue survival has been shown to be active and upregulated in frozen wood frog brains, emphasizing the importance of maintaining brain functionality (Aguilar et al., 2016). One of the main questions we sought to investigate is, what happens 
to the brains of freeze tolerant animals? Indeed, while it has been reported that brains from frozen wood frogs display no measurable brain activity and both visual observations and magnetic resonance imaging have shown ice accumulation in brain ventricles, little other physiological work has been done on freeze tolerant brains (Storey and Storey, 2017). In the present study, we investigated the regulation of microRNA biogenesis and the expression levels of 113 miRNAs over the course of a freeze-thaw cycle in wood frog brains. This lead to the identification of a global pattern of downregulation of both miRNA biogenesis and relative expression levels. This freeze-thaw specific miRNA fingerprint of downregulation suggests that wood frog brains may utilize miRNAs in a cryoprotective manner to maintain neural networks and circumvent extensive transcriptional and translational repression.

Recent studies have demonstrated that the activity, function, and abundance of miRNAprotein complexes can alter miRNA biogenesis via the regulation of environmental and stress-responsive signalling pathways (Leung and Sharp, 2010). An examination of the miRNA biogenesis and processing proteins revealed an overall pattern of downregulation over the freeze-thaw cycle (Fig. 3.1). As the efficiency of DROSHA-mediated processing is crucial for determining miRNA abundance, the observed reduction of microprocessor protein levels suggests a freeze-induced reduction in initial miRNA maturation and premiRNA synthesis that could be a result of the energy limitations imposed during freezing and thawing (Tang et al., 2013). Furthermore, reduced levels of EXPORTIN-5 have been shown to not only lead to reductions in cytoplasmic miRNA levels, but also to pre-miRNA nucleolytic degradation in the nucleus (Fig. 3.1) (Yi et al., 2003). However, this warrants further investigation as EXPORTIN-5 functions in a GTP-dependant fashion and protein 
levels of the RAN GTP-binding protein, that complexes with EXPORTIN-5 to transport pre-miRNAs into the cytoplasm, remained constant (Fig. 3.1) (Bohnsack et al., 2004; Okada et al., 2009). Following nuclear export, nascent pre-miRNAs are cleaved into mature miRNA duplexes by DICER's endonuclease activity and while DICER levels remained constant during both freezing and thawing, protein levels of two other components of the RISC complex, TRBP and PACT, were downregulated during thawing and freezing, respectively (Fig. 3.1). Indeed, reduced levels of TRBP and PACT lower the efficiency of post-transcriptional gene silencing and the depletion of TRBP could play a role in DICER destabilization and miRNA downregulation (Ha and Kim, 2014; Winter et al., 2009). However, it should be noted that TRBP and PACT are non-essential facilitators of DICER-mediated pre-miRNA processing (Lee et al., 2006).

The miRNA guide strand is then loaded onto an Argonaute protein effector complex, and while levels of AGO1, that is known to interact with only $\sim 30 \%$ of miRNAs, remained unchanged, protein levels of AGO2, that interacts with $\sim 60 \%$ of all miRNAs, were downregulated over the freeze-thaw cycle (Wang et al., 2012) (Fig. 3.1). AGO2 is the sole Argonaute with slicer activity and the loss of $\mathrm{AGO} 2$ has been demonstrated to result in the reduction of mature miRNA expression and activity (Winter et al., 2009). However, levels of $\mathrm{p}-\mathrm{AGO} 2^{\mathrm{Ty} 393}$ were downregulated during thawing which contradicted the overall observed suppression of miRNA biogenesis as this phosphorylation, commonly associated with hypoxic conditions, has been shown to result in the inhibition of miRNA processing and DICER binding (Shen et al., 2013) (Fig. 3.1). Indeed, AGO2 is a prime candidate for the regulation of miRNA biogenesis and function and our findings suggest that there likely is diminished mature mRNA translational inhibition, destabilization, and/or target 
degradation. While our findings suggest an overall reduction in miRNA biogenesis and processing, especially during the nuclear maturation steps, future investigation should also explore regulation of the less common non-canonical miRNA biogenesis mechanisms such as the DROSHA and DGCR8-independent pathway, the DICER-independent pathway, and the terminal uridylyl transferase dependant pathway which may be used to circumvent the reduced levels of these miRNA biogenesis factors (Ha and Kim, 2014).

Initial studies on frozen wood frog miRNAs examined the regulation of miR-16 and miR21 in liver and skeletal muscle and 53 miRNA species in cardiac and skeletal muscle over the course of a freeze-thaw cycle (Bansal et al., 2016; Biggar et al., 2009). The present large-scale study provides us with a better understanding of the cellular mechanisms that contribute to successful brain freeze tolerance. During freezing, 23 of the 113 miRNA species analyzed in brain were found to be significantly downregulated while $r s y-m i R-451$ $5 p$ was the only miRNA found to be freeze-upregulated (Fig. 3.2). Previous work on miR451 has shown this unique miRNA to act as a conditional glucose sensing switch, whereby high glucose levels result in its overexpression, which in turn leads to the suppression of downstream protein kinases and the PI3K/AKT pathway, resulting in unrestrained mTOR activation (Godlewski et al., 2010; Tian et al., 2012). Indeed, during freezing, the brain, and all wood frog organs, are flooded with large amounts of cryoprotectant glucose that protect against mechanical ice damage and excessive cellular dehydration (Storey and Storey, 2017). The upregulation of $r s y-m i R-451-5 p$ could be a result of abundant glucose levels but could also be involved in maintaining neural functions during freezing. In addition, miR-451 has also been shown to protect against anoxia/reoxygenation injury, a 
stress that frozen wood frog brains are vulnerable to, through the inhibition of $14-3-3 \zeta$ and high mobility group box 1 expression, respectively (Xie et al., 2016; Yu et al., 2010).

While the majority of miRNA species measured exhibited constant expression levels over the freeze-thaw cycle, 23 of the differentially expressed miRNAs were found to be significantly downregulated during freezing (Fig. 3.2). Predicted targets of the freezedownregulated miRNAs were collectively analyzed and found to potentially be involved in maintaining cellular processes such as intracellular signal transduction, RNA processing, and synaptic signalling, among others (Fig. 3.3). The reduced levels of these differentially expressed miRNAs implies that the gene transcripts under their control are more likely to be translated, which in turn implies that the protein product the gene encodes for will be expressed and may play a role in facilitating freeze tolerance. The various biological processes targeted by the downregulated miRNAs appear to be involved in the maintenance of basic neuronal cell function and survival. Targeting of proteins essential for neurotransmitter release and pre-synaptic functions such as synaptosomal-associated protein of $25 \mathrm{kDa}(\mathrm{SNAP} 25)$ suggests an enhancement of their roles during freezing, possibly in a protective manner. Downregulated miRNAs 103/107-5p, 10b-5p, 183-5p, 184-3p, 18-5p, 193-3p, 196b-5p, 210-3p, 214-3p, 2970-5p, 449b-5p (Supp. Table S3.3) were predicted to target SNAP25, a known pre-synaptic reactive oxygen species (ROS) sensor that exhibits ROS-induced functional impairment. This suggests that its predicted upregulation during freezing and thawing could be in an effort to minimize impairment and promote synaptic vesicle priming and signalling (Giniatullin et al., 2006).

Interestingly, a similar activation of long-term potentiation and axon guidance were reported in other miRNA studies of brains of other hypometabolic survival strategies to 
environmental stress including mammalian hibernation and frog estivation (Biggar et al., 2014; Luu and Storey, 2015). Interestingly, this pattern of downregulation and the promotion of neuroprotective mechanisms has also been reported in whole brains of another environmentally stressed frog, the estivating African-clawed frog, where 12 of the 43 miRNAs measured exhibited a significant downregulation (Luu and Storey, 2015). The downregulation of miRNAs in the brains of environmentally stressed anurans may be an evolutionarily conserved response. Indeed, while freezing is a unique physiological response, it builds on and exaggerates many pre-existing strategies that stem from the ability of anurans to survive dehydration as dehydration is one of the main component stresses of freezing (Costanzo et al., 1993; Storey and Storey, 2017). These results suggest that these miRNAs are involved in regulating brain neural plasticity. Despite the fact that wood frogs display no measurable brain activity during freezing, upon thawing, frogs are able to immediately resume normal functions with no apparent neurological damage, loss of pre-existing memories, or deleterious behavioural changes. We therefore propose the existence of a miRNA-mediated neuroprotective mechanism to circumvent the degeneration of neural synapses and for the protection against the negative effects of prolonged states of hypometabolic dormancy. Indeed, our data suggest that rather than modulating metabolic rate depression, the differentially expressed miRNAs in frozen wood frog brains appear to be responsible for neural network plasticity and maintenance. Our findings point away from a miRNA-mediated mechanism for a freeze-induced state of translational suppression but rather towards a role for miRNAs as tuners of neuronal maintenance and function. 
Thawing exhibited a similar but more pronounced response to that of freezing, with the downregulation of 33 miRNAs and the upregulation of rsy-miR-192-5p (Fig. 3.2 and Supp. Table S3.2). Bioinformatic target enrichment implicated the downregulated miRNAs in the predicted activation of numerous brain-specific cytoprotective processes such as chaperone-mediated protein folding, synaptic signalling, and intracellular signal transduction (Fig. 3.4 and Supp. Table S3.4). Protein folding networks are a critical cell preservation strategy essential for ensuring protein homeostasis via the proper assembly of new proteins, the prevention of their aggregation, and the promotion of their efficient folding (Hartl et al., 2011). Molecular chaperones have been shown to be cold/freeze upregulated in cold hardy arthropods and in oxygen-limited states including those experienced by anoxia tolerant turtles (Jiang et al., 2016; Ramaglia and Buck, 2004; Storey and Storey, 2011, 2012). Furthermore, the importance of chaperones in mediating successful freeze-thaw has also been demonstrated in yeast, where the deletion of chaperones resulted in reduced cell viability to freeze-thaw events (Naicker et al., 2012). As such, the predicted activation of chaperone-mediated protein folding in the brains of thawing wood frogs through the upregulation of heat shock proteins Hsp10 and Hsp60 mitochondrial chaperones, and other proteins in the network (Fig. 3.4) underscores the involvement of stress-induced chaperone expression in protecting nervous system tissues. Interestingly, a recent study on zebrafish has shown that injection of Hsp60 triggers tissue regeneration and wound healing, another possible role for the protein during thawing (Pei et al., 2016).

The observed thaw-induced downregulation of $r s y-m i R-181 a-3 p$ could potentially serve a similar function to the role it plays in the brains of ischemic mice, where it was found to 
promote long lasting brain recovery via its action as an apoptotic modulator capable of inducing the upregulation of apoptosis-inhibiting and pro-survival proteins, XIAP and BCL2, respectively (Moon et al., 2013; Xu et al., 2015). Indeed, BCL2 has been shown to play a central role in the regulation of neuronal cell survival and neurodegeneration and is a known target of miR-204, that was found to be downregulated during both freezing and thawing (Akhtar et al., 2004; Sacconi et al., 2012) (Fig. 3.2). In addition, miR-210, that was downregulated during both freezing and thawing, was also shown to provide neuroprotection against hypoxic/ischemic brain injury, as its suppression has been associated with increased levels of glucocorticoid receptors which resulted in improvement to long-term neurological function recovery and reduced brain infarct size (Ma et al., 2016). However, despite this pattern of downregulation observed during thawing, $r s y-m i R$ 192-5p was identified as the sole thaw-upregulated miRNA (Fig. 3.2). The enhanced expression of miR-192 has been shown to be tightly associated with Smad3, where Smad3 is able to mediate TGF- $\beta 1$-induced miR-192 overexpression by binding to, and activating the miR-192 promoter (Chung et al., 2010). Interestingly, this corroborates the aforementioned significant upregulation of $\mathrm{p}$-Smad3 protein levels in $R$. sylvatica brains during both freezing, and to a larger extent during thawing (Aguilar et al., 2016; Chung et al., 2010). A known target of miR-192, that could be targeted in thawed brains, is Semaphorin 3A, the suggested miR-192 mediated silencing of Semaphorin 3A could be an ischemia protection mechanism. Indeed, elevated Semaphorin 3A have been shown to be a critical facilitator of cerebrovascular permeability and ischemia-induced brain damage and as such its silencing should aid in protecting frogs from the ischemic damages associates with thawing (Hou et al., 2015; Yan-Chun et al., 2017). 
Taken together, the miRNA biogenesis and relative expression findings suggest that the global reduction in miRNA biogenesis proteins during both freezing and thawing may be facilitating the overall downregulation of differentially expressed miRNAs (Fig. 3.1 and Fig. 3.2). Many of the downregulated miRNAs appear to be involved in providing neuroprotection against hypoxic/ischemic brain injury during both freezing and thawing. Indeed, the downregulation of the plasticity-related miR-24 over the freeze-thaw cycle, has been linked with the induction and persistence of long-term potentiation through the rapid release of target mRNA transcript inhibition (Ryan et al., 2012). Furthermore, numerous pathways such as intracellular signal transduction were found to be targeted during both freezing and thawing, suggesting a consistent enhancement of their regulatory functions over the course of the freeze-thaw cycle (Fig. 3.3 and 3.4). These processes are involved in neuronal maintenance and could act to better adapt neurons to environmental cues and protect delicate brain tissue from freeze-thaw induced damages. Since miRNA expression in brains is region-specific, neuron type-specific, and cell compartment-specific (O’Carroll and Schaefer, 2013), further dissecting the observed whole-brain miRNA fingerprint may provide us with additional insights into the miRNA involvement of these various factors. 


\subsection{CONCLUSION}

Our findings demonstrate, for the first time, that wood frog brains appear to circumvent the transcriptional and translational repression that is typical of other organs, possibly to sustain brain function though prolonged periods of environmental stress. The correlation between the downregulation of miRNA biogenesis factor levels and the differentially expressed miRNAs over the freeze-thaw cycle emphasizes the importance of brain function for the overall adaptation of the animal to freezing. Indeed, the downregulated miRNAs appear to impart neuroprotective functions by targeting pathways involved in neuronal maintenance, survival, and anoxia/ischemia protective mechanisms. These results suggest that the 41 differentially expressed miRNAs are not only freeze-thaw responsive but are also involved in maintaining brain function and protecting against freeze-associated damages. Taken together, this study provides us with insights into the molecular underpinnings of neuronal adaptations to environmental changes and the mechanisms to facilitate natural freeze tolerance in vertebrate central nervous systems.

\section{ACKNOWLEDGEMENTS}

This work was supported by a Discovery grant (Grant \# 6793) from the Natural Sciences and Engineering Research Council (NSERC) of Canada. KBS holds the Canada Research Chair in Molecular Physiology and HH holds a NSERC Postgraduate scholarship.

\section{CONFLICT OF INTEREST}

The authors declare no conflict of interest. 

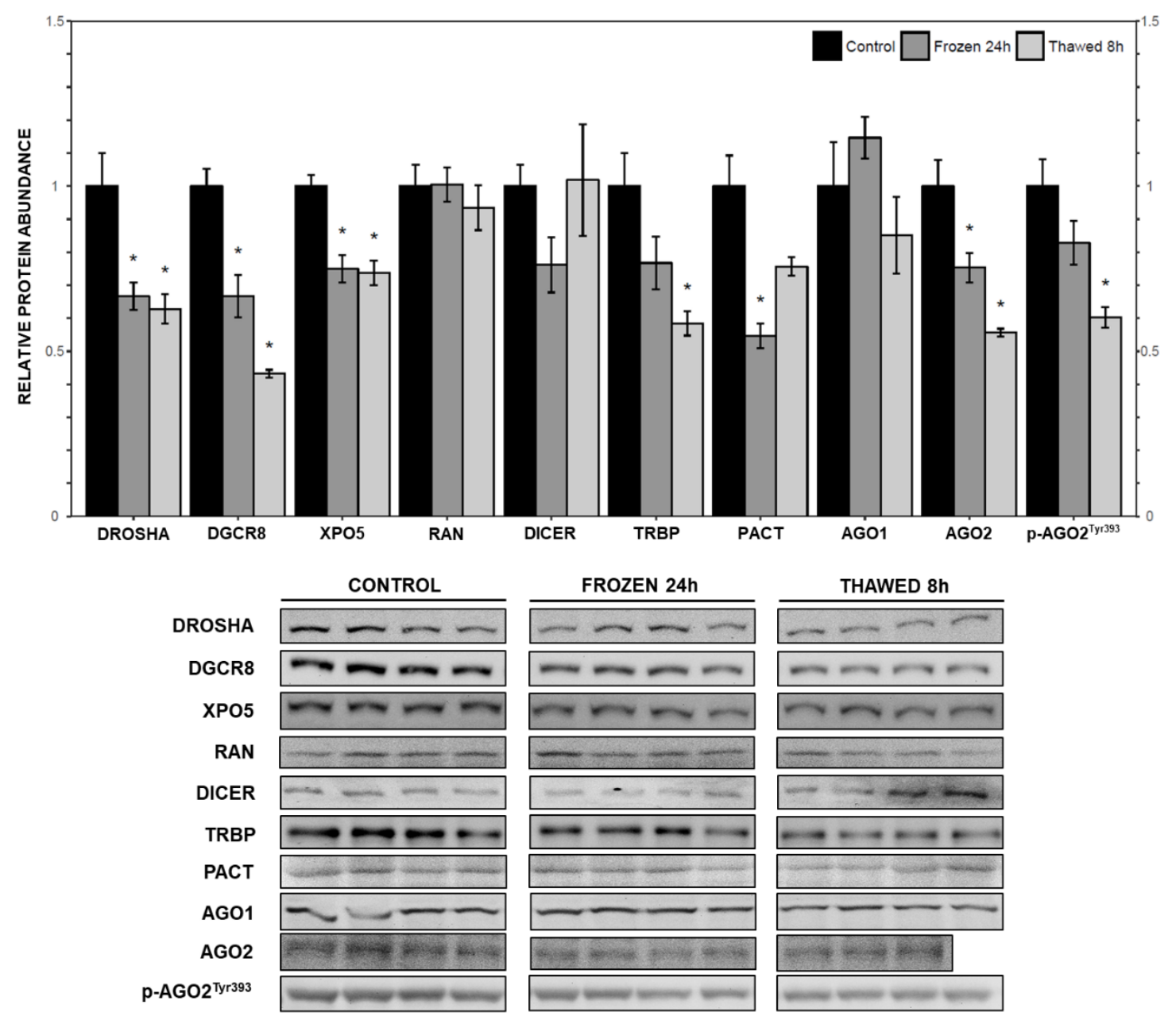

Figure 3.1. Analysis of miRNA biogenesis pathway protein levels in wood frog brain over a freeze-thaw cycle using immunoblotting. Upper panel displays the histogram showing protein levels, relative to control, of DROSHA, DGCR8, XPO5, RAN, DICER, TRBP, PACT, AGO1, AGO2, and p-AGO2 ${ }^{\text {Tyr393 }}$ under control, $24 \mathrm{~h}$ frozen, and $8 \mathrm{~h}$ thawed conditions. Data are means \pm SEM of 3-4 independent biological replicates, relative to control values. Data are analyzed using a one-way ANOVA with a Dunnett's post hoc test, $* p<0.05$. Bottom panel show representative immunoblots of each protein and experimental condition. 


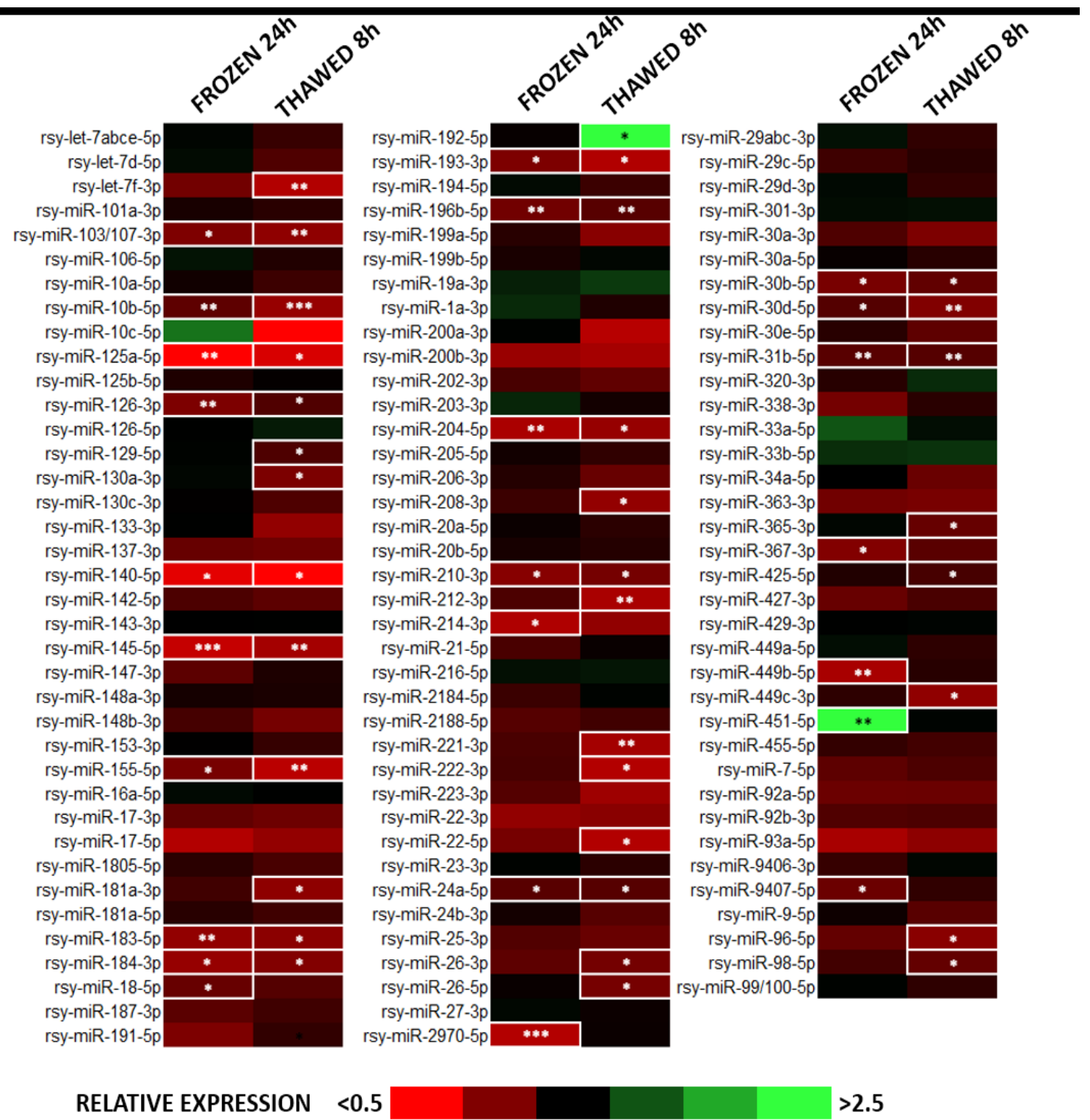

Figure 3.2. Heatmap of RT-qPCR measured expression levels of 113 miRNA species examined in $24 \mathrm{~h}$ frozen and $8 \mathrm{~h}$ thawed wood frog brain, relative to control. All miRNAs were standardized against Snord68 reference gene expression. Data are means \pm SEM of 3-4 independent biological replicates. Statistical testing used a one-way ANOVA with a Dunnett's post hoc test, ${ }^{*} p<0.05$. For the relative expression \pm SEM values of all 113 miRNA species examined, refer to Supplementary Table S2. 
Figure 3.3. Functional target enrichment and network clustering of the subset of miRNAs downregulated in the brains of $24 \mathrm{~h}$ frozen wood frogs. Downstream miRNA target prediction was performed at $-2^{\circ} \mathrm{C}$ using FINDTAR3. Protein-protein interactions of the downstream networks was performed using the STRING high-confidence filter on the $X$. tropicalis database. MCL clustering and visualization was performed on CYTOSCAPE and coupled with functional biological enrichment using GO ANALYSIS. The full cluster map is illustrated on the right and significantly enriched representative clusters are highlighted on the left. Refer to Supplementary Table S3.3 for more information on individual clusters, proteins, and the targeting of individual miRNA species. 


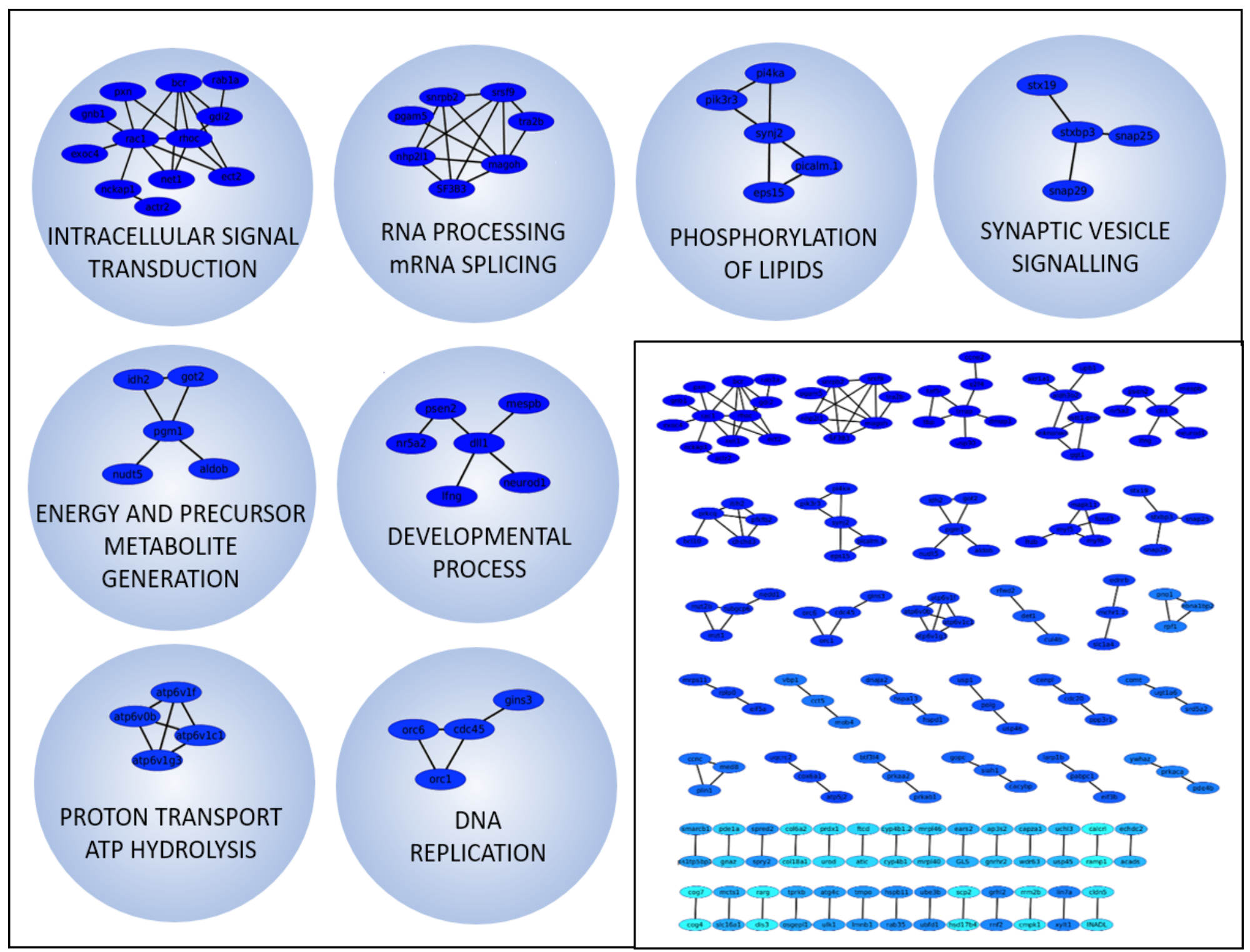


Figure 3.4. Functional target enrichment and network clustering of the subset of miRNAs downregulated in the brains of $8 \mathrm{~h}$ thawed wood frogs. Downstream miRNA target prediction was performed at $5^{\circ} \mathrm{C}$ using FINDTAR3. Protein-protein interactions of the downstream networks was performed using the STRING high-confidence filter on the $X$. tropicalis database. MCL clustering and visualization was performed on CYTOSCAPE and coupled with functional biological enrichment using GO ANALYSIS. The full cluster map is illustrated on the right and significantly enriched representative clusters are highlighted on the left. Refer to Supplementary Table S3.4 for more information on individual clusters, proteins, and the targeting of individual miRNA species. 


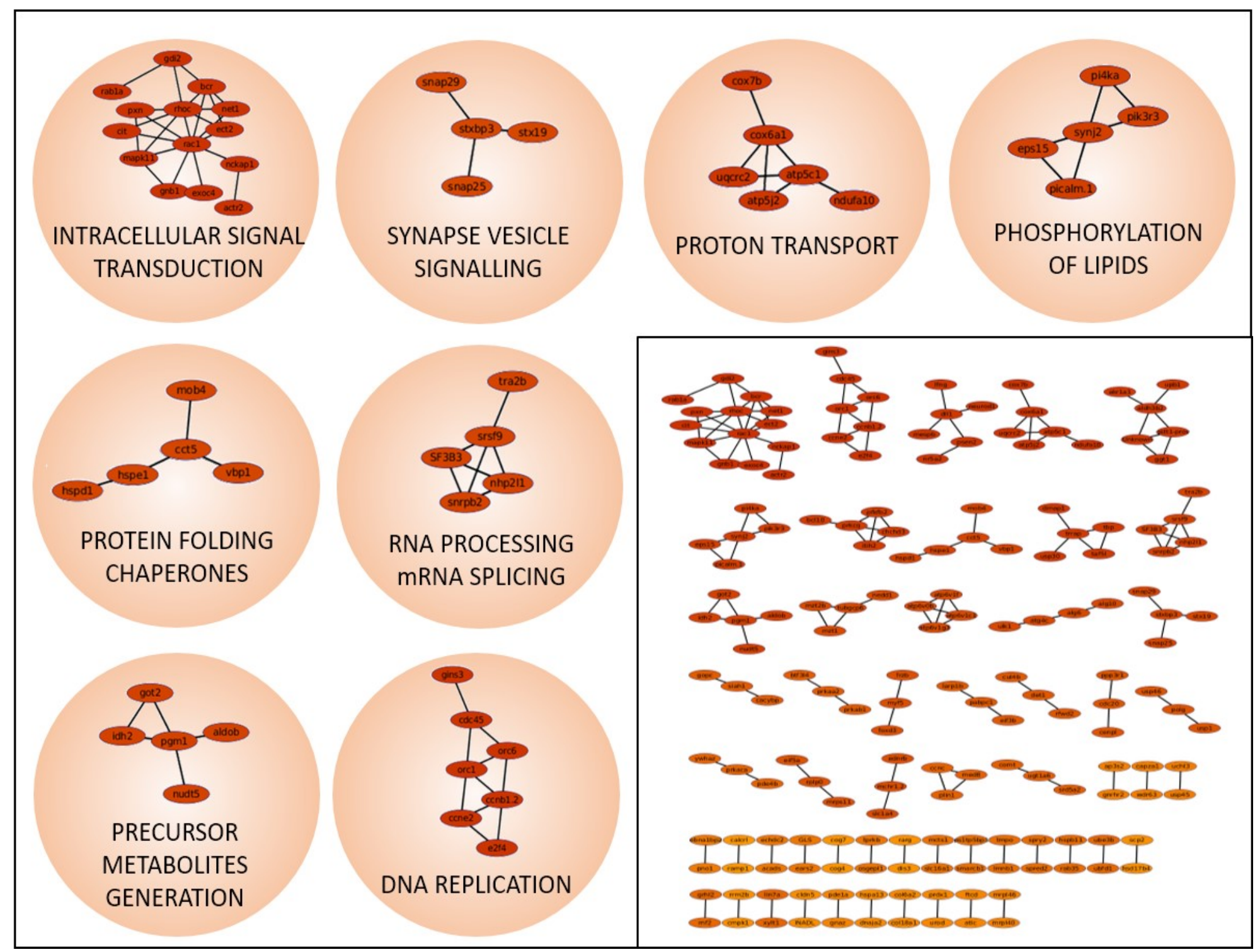




\section{SUPPLEMNTARY INFORMATION}

To access and download the four additional supplementary files that accompany this manuscript:

1. Visit: http://kenstoreylab.com/?page $\mathrm{id}=2899$

2. Enter the password: HananeWantsToPass [*CaSe SeNsiTivE]

Supplementary Table S3.1. Primers used for analysis of miRNA expression in the brain of $R$. sylvatica, including miRNA-specific forward primers, universal reverse primer, and the stem-loop adapter for reverse-transcription.

Supplementary Table S3.2. Relative expression levels of 113 miRNA species examined in the brain of $R$. sylvatica. MicroRNA relative expression was evaluated by RT-qPCR of reverse-transcribed, polyadenylated transcripts. Data represent means of $n=3-4$ biological replicates from different animals \pm SEM. Relative expression of genes was calculated by standardizing against snord68 expression. Control values were adjusted to 1 and the $24 \mathrm{~h}$ frozen and $8 \mathrm{~h}$ thawed values were expressed relative to the control. Statistical testing used the Dunnett's test, values were considered significantly different from the corresponding control when $* p<0.05, * * p<0.01$, and $* * * p<0.001$.

Supplementary Table S3.3. Identity of the significantly enriched biological processes, protein members, and miRNA species identified using MCL clustered protein networks and functional GO ANALYSIS of the miRNAs downregulated in $24 \mathrm{~h}$ frozen frog brain.

Supplementary Table S3.4. Identity of the significantly enriched biological processes, protein members, and miRNA species identified using MCL clustered protein networks and functional GO ANALYSIS of the miRNAs downregulated in $8 \mathrm{~h}$ thawed frog brain. 


\section{REFERENCES [CHAPTER 3]}

Abe, M., Bonini, N.M., 2013. MicroRNAs and neurodegeneration: role and impact. Trends Cell Biol. 23, 30-6.

Aguilar, O.A., Hadj-Moussa, H., Storey, K.B., 2017. Freeze-responsive regulation of MEF2 proteins and downstream gene networks in muscles of the wood frog, Rana sylvatica. J. Therm. Biol.

Aguilar, O.A., Hadj-Moussa, H., Storey, K.B., 2016. Regulation of SMAD transcription factors during freezing in the freeze tolerant wood frog, Rana sylvatica. Comp. Biochem. Physiol. B. Biochem. Mol. Biol. 201, 64-71.

Akhtar, R.S., Ness, J.M., Roth, K.A., 2004. Bcl-2 family regulation of neuronal development and neurodegeneration. Biochim. Biophys. Acta - Mol. Cell Res. 1644, 189-203.

Bansal, S., Luu, B.E., Storey, K.B., 2016. MicroRNA regulation in heart and skeletal muscle over the freeze-thaw cycle in the freeze tolerant wood frog. J. Comp. Physiol. B 186, 229-241.

Bartel, D.P., 2009. MicroRNAs: Target recognition and regulatory functions. Cell 136, 215-233.

Biggar, K.K., Dubuc, A., Storey, K., 2009. MicroRNA regulation below zero: Differential expression of miRNA-21 and miRNA-16 during freezing in wood frogs. Cryobiology 59, 317-321.

Biggar, K.K., Kornfeld, S.F., Maistrovski, Y., Storey, K.B., 2012. MicroRNA Regulation in Extreme Environments: Differential Expression of MicroRNAs in the Intertidal Snail Littorina littorea During Extended Periods of Freezing and Anoxia. Genomics. Proteomics Bioinformatics 10, 302-309.

Biggar, K.K., Storey, K.B., 2015. Low-temperature microRNA expression in the painted turtle, Chrysemys picta during freezing stress. FEBS Lett. 589, 3665-3670.

Biggar, K.K., Storey, K.B., 2014. Insight into temperature-dependent microRNA function in mammalian hibernators. Temperature 1, 84-86.

Biggar, K.K., Storey, K.B., 2011. The emerging roles of microRNAs in the molecular responses of metabolic rate depression. J. Mol. Cell Biol. 3, 167-175.

Biggar, K.K., Wu, C.-W., Storey, K.B., 2014. High-throughput amplification of mature microRNAs in uncharacterized animal models using polyadenylated RNA and stem-loop reverse transcription polymerase chain reaction. Anal. Biochem. 462, 3234.

Bohnsack, M.T., Czaplinski, K., Gorlich, D., 2004. Exportin 5 is a RanGTP-dependent dsRNA-binding protein that mediates nuclear export of pre-miRNAs. RNA 10, 185- 
91.

Cai, Q., Storey, K.B., 1997. Upregulation of a novel gene by freezing exposure in the freeze-tolerant wood frog (Rana sylvatica). Gene 198, 305-12.

Chen, M., Storey, K.B., 2014. Large-scale identification and comparative analysis of miRNA expression profile in the respiratory tree of the sea cucumber Apostichopus japonicus during aestivation. Mar. Genomics 13, 39-44.

Chung, A.C.K., Huang, X.R., Meng, X., Lan, H.Y., 2010. miR-192 mediates TGFbeta/Smad3-driven renal fibrosis. J. Am. Soc. Nephrol. 21, 1317-25.

Costanzo, J.P., Lee, R.E., Lortz, P.H., 1993. Physiological responses of freeze-tolerant and -intolerant frogs: clues to evolution of anuran freeze tolerance. Am. J. Physiol. 265, R721-5.

Costanzo, J.P., Lee, R.E., Ultsch, G.R., 2008. Physiological ecology of overwintering in hatchling turtles. J. Exp. Zool. Part A Ecol. Genet. Physiol. 309A, 297-379.

Dawson, N.J., Katzenback, B.A., Storey, K.B., 2015. Free-radical first responders: The characterization of $\mathrm{CuZnSOD}$ and MnSOD regulation during freezing of the freezetolerant North American wood frog, Rana sylvatica. Biochim. Biophys. Acta - Gen. Subj. 1850, 97-106.

Denlinger, D.L., Lee, R.E., 2010. Low temperature biology of insects. Cambridge University Press.

Dieni, C.A., Storey, K.B., 2014. Protein kinase C in the wood frog, Rana sylvatica: reassessing the tissue-specific regulation of $\mathrm{PKC}$ isozymes during freezing. PeerJ 2 , e558.

Ebert, M.S., Sharp, P.A., 2012. Roles for microRNAs in conferring robustness to biological processes. Cell 149, 515-24.

Fukunaga, R., Han, B.W., Hung, J.-H., Xu, J., Weng, Z., Zamore, P.D., 2012. Dicer Partner Proteins Tune the Length of Mature miRNAs in Flies and Mammals. Cell $151,533-546$.

Giniatullin, A.R., Darios, F., Shakirzyanova, A., Davletov, B., Giniatullin, R., 2006. SNAP25 is a pre-synaptic target for the depressant action of reactive oxygen species on transmitter release. J. Neurochem. 98, 1789-1797.

Godlewski, J., Bronisz, A., Nowicki, M.O., Chiocca, E.A., Lawler, S., 2010. microRNA451: A conditional switch controlling glioma cell proliferation and migration. Cell Cycle 9, 2742-8.

Greenway, S.C., Storey, K.B., 2000. Activation of mitogen-activated protein kinases during natural freezing and thawing in the wood frog. Mol. Cell. Biochem. 209, 2937. 
Ha, M., Kim, V.N., 2014. Regulation of microRNA biogenesis. Nat. Rev. Mol. Cell Biol.

Hadj-Moussa, H., Moggridge, J.A., Luu, B.E., Quintero-Galvis, J.F., Gaitán-Espitia, J.D., Nespolo, R.F., Storey, K.B., 2016. The hibernating South American marsupial, Dromiciops gliroides, displays torpor-sensitive microRNA expression patterns. Sci. Rep. 6, 24627.

Hartl, F.U., Bracher, A., Hayer-Hartl, M., 2011. Molecular chaperones in protein folding and proteostasis. Nature 475, 324-332.

Holmstrup, M., 2014. The ins and outs of water dynamics in cold tolerant soil invertebrates. J. Therm. Biol. 45, 117-123.

Hou, S.T., Nilchi, L., Li, X., Gangaraju, S., Jiang, S.X., Aylsworth, A., Monette, R., Slinn, J., 2015. Semaphorin3A elevates vascular permeability and contributes to cerebral ischemia-induced brain damage. Sci. Rep. 5, 7890.

Huang, Y., Shen, X.J., Zou, Q., Wang, S.P., Tang, S.M., Zhang, G.Z., 2011. Biological functions of microRNAs: a review. J. Physiol. Biochem. 67, 129-139.

Jaskiewicz, L., Filipowicz, W., 2008. Role of Dicer in posttranscriptional RNA silencing. Curr. Top. Microbiol. Immunol. 320, 77-97.

Jiang, J.-J., Xia, E.-H., Gao, C.-W., Gao, L.-Z., 2016. The complete mitochondrial genome of western painted turtle, Chrysemys picta bellii (Chrysemys, Emydidae). Mitochondrial DNA 27, 787-788.

Lee, H.Y., Doudna, J.A., 2012. TRBP alters human precursor microRNA processing in vitro. RNA 18, 2012-2019.

Lee, Y., Hur, I., Park, S.-Y., Kim, Y.-K., Suh, M.R., Kim, V.N., 2006. The role of PACT in the RNA silencing pathway. EMBO J. 25, 522-532.

Leung, A.K.L., Sharp, P.A., 2010. MicroRNA functions in stress responses. Mol. Cell 40, 205-215.

Luu, B.E., Storey, K.B., 2015. Dehydration triggers differential microRNA expression in Xenopus laevis brain. Gene.

Lyons, P.J., Crapoulet, N., Storey, K.B., Morin, P.J., 2015. Identification and profiling of miRNAs in the freeze-avoiding gall moth Epiblema scudderiana via next-generation sequencing. Mol. Cell. Biochem. 410, 155-163.

Lyons, P.J., Lang-Ouellette, D., Morin, P.J., 2013. CryomiRs: Towards the identification of a cold-associated family of microRNAs. Comp. Biochem. Physiol. Part D Genomics Proteomics 8, 358-364.

Ma, Q., Dasgupta, C., Li, Y., Bajwa, N.M., Xiong, F., Harding, B., Hartman, R., Zhang, L., 2016. Inhibition of microRNA-210 provides neuroprotection in hypoxicischemic brain injury in neonatal rats. Neurobiol. Dis. 89, 202-212. 
MacFarlane, L.-A., Murphy, P.R., 2010. MicroRNA: Biogenesis, Function and Role in Cancer. Curr. Genomics 11, 537-561.

Moon, J., Xu, L., Giffard, R.G., 2013. Inhibition of microRNA-181 reduces forebrain ischemia-induced neuronal loss. J. Cereb. Blood Flow Metab. 33, 1976-82.

Naicker, M.C., Seul Jo, I., Im, H., 2012. Identification of chaperones in freeze tolerance in Saccharomyces cerevisiae. J. Microbiol. 50, 882-887.

O'Carroll, D., Schaefer, A., 2013. General principals of miRNA biogenesis and regulation in the brain. Neuropsychopharmacology 38, 39-54.

Okada, C., Yamashita, E., Lee, S.J., Shibata, S., Katahira, J., Nakagawa, A., Yoneda, Y., Tsukihara, T., 2009. A high-resolution structure of the pre-microRNA nuclear export machinery. Science 326, 1275-9.

Pei, W., Tanaka, K., Huang, S.C., Xu, L., Liu, B., Sinclair, J., Idol, J., Varshney, G.K., Huang, H., Lin, S., Nussenblatt, R.B., Mori, R., Burgess, S.M., 2016. Extracellular HSP60 triggers tissue regeneration and wound healing by regulating inflammation and cell proliferation. npj Regen. Med. 1, 16013.

Ramaglia, V., Buck, L.T., 2004. Time-dependent expression of heat shock proteins 70 and 90 in tissues of the anoxic western painted turtle. J. Exp. Biol. 207, 3775-3784.

Ryan, M.M., Ryan, B., Kyrke-Smith, M., Logan, B., Tate, W.P., Abraham, W.C., Williams, J.M., 2012. Temporal profiling of gene networks associated with the late phase of long-term potentiation in vivo. PLoS One 7, e40538.

Sacconi, A., Biagioni, F., Canu, V., Mori, F., Di Benedetto, A., Lorenzon, L., Ercolani, C., Di Agostino, S., Cambria, A.M., Germoni, S., Grasso, G., Blandino, R., Panebianco, V., Ziparo, V., Federici, O., Muti, P., Strano, S., Carboni, F., Mottolese, M., Diodoro, M., Pescarmona, E., Garofalo, A., Blandino, G., 2012. miR-204 targets Bcl-2 expression and enhances responsiveness of gastric cancer. Cell Death Dis. 3, e423.

Schmittgen, T.D., Livak, K.J., 2008. Analyzing real-time PCR data by the comparative CT method. Nat. Protoc. 3, 1101-1108.

Shannon, P., Markiel, A., Ozier, O., Baliga, N.S., Wang, J.T., Ramage, D., Amin, N., Schwikowski, B., Ideker, T., 2003. Cytoscape: A software environment for integrated models of biomolecular interaction networks. Genome Res. 13, 2498 2504 .

Shen, J., Xia, W., Khotskaya, Y.B., Huo, L., Nakanishi, K., Lim, S.-O., Du, Y., Wang, Y., Chang, W.-C., Chen, C.-H., Hsu, J.L., Wu, Y., Lam, Y.C., James, B.P., Liu, X., Liu, C.-G., Patel, D.J., Hung, M.-C., 2013. EGFR modulates microRNA maturation in response to hypoxia through phosphorylation of AGO2. Nature 497, 383-7.

Storey, K., Storey, J.M., 2011. Heat shock proteins and hypometabolism: adaptive 
strategy for proteome preservation. Res. Rep. Biol. 2, 57-68.

Storey, K.B., 2015. Regulation of hypometabolism: insights into epigenetic controls. J. Exp. Biol. 218.

Storey, K.B., 2006. Reptile freeze tolerance: Metabolism and gene expression. Cryobiology 52, 1-16.

Storey, K.B., 1990. Life in a frozen state: adaptive strategies for natural freeze tolerance in amphibians and reptiles. Am. J. Physiol. 258, R559-68.

Storey, K.B., Storey, J.M., 2017. Molecular physiology of freeze tolerance in vertebrates. Physiol. Rev. 97, 623-665.

Storey, K.B., Storey, J.M., 2012. Insect cold hardiness: metabolic, gene, and protein adaptation. Can. J. Zool. 90, 456-475.

Storey, K.B., Storey, J.M., 2007. Tribute to P. L. Lutz: putting life on 'pause' - molecular regulation of hypometabolism. J. Exp. Biol. 210.

Storey, K.B., Storey, J.M., 2004. Metabolic rate depression in animals: transcriptional and translational controls. Biol. Rev. Camb. Philos. Soc. 79, 207-33.

Strimbeck, G.R., Schaberg, P.G., Fossdal, C.G., Schröder, W.P., Kjellsen, T.D., 2015. Extreme low temperature tolerance in woody plants. Front. Plant Sci. 6, 884.

Sullivan, K.J., Storey, K.B., 2012. Environmental stress responsive expression of the gene li16 in Rana sylvatica, the freeze tolerant wood frog. Cryobiology 64, 192200.

Szklarczyk, D., Franceschini, A., Wyder, S., Forslund, K., Heller, D., Huerta-Cepas, J., Simonovic, M., Roth, A., Santos, A., Tsafou, K.P., Kuhn, M., Bork, P., Jensen, L.J., von Mering, C., 2015. STRING v10: protein-protein interaction networks, integrated over the tree of life. Nucleic Acids Res. 43, D447-D452.

Tang, X., Wen, S., Zheng, D., Tucker, L., Cao, L., Pantazatos, D., Moss, S.F., Ramratnam, B., 2013. Acetylation of Drosha on the N-Terminus Inhibits Its Degradation by Ubiquitination. PLoS One 8, e72503.

Tian, Y., Nan, Y., Han, L., Zhang, A., Wang, G., Jia, Z., Hao, J., Pu, P., Zhong, Y., Kang, C., 2012. MicroRNA miR-451 downregulates the PI3K/AKT pathway through CAB39 in human glioma. Int. J. Oncol. 40, 1105-12.

Wang, D., Zhang, Z., O’Loughlin, E., Lee, T., Houel, S., O'Carroll, D., Tarakhovsky, A., Ahn, N.G., Yi, R., 2012. Quantitative functions of Argonaute proteins in mammalian development. Genes Dev. 26, 693-704.

Winter, J., Jung, S., Keller, S., Gregory, R.I., Diederichs, S., 2009. Many roads to maturity: microRNA biogenesis pathways and their regulation. Nat. Cell Biol. 11, $228-234$. 
Wu, P., Nakano, S., Sugimoto, N., 2002. Temperature dependence of thermodynamic properties for DNA/DNA and RNA/DNA duplex formation. Eur. J. Biochem. 269, 2821-30.

Wu, S., De Croos, J.N.A., Storey, K.B., 2008. Cold acclimation-induced up-regulation of the ribosomal protein $\mathrm{L} 7$ gene in the freeze tolerant wood frog, Rana sylvatica. Gene $424,48-55$.

Wu, S., Storey, K.B., 2005. Up-regulation of acidic ribosomal phosphoprotein P0 in response to freezing or anoxia in the freeze tolerant wood frog, Rana sylvatica. Cryobiology 50, 71-82.

Xie, J., Hu, X., Yi, C., Hu, G., Zhou, X., Jiang, H., 2016. MicroRNA-451 protects against cardiomyocyte anoxia/reoxygenation injury by inhibiting high mobility group box 1 expression. Mol. Med. Rep. 13, 5335-41.

Xu, L.-J., Ouyang, Y.-B., Xiong, X., Stary, C.M., Giffard, R.G., 2015. Post-stroke treatment with miR-181 antagomir reduces injury and improves long-term behavioral recovery in mice after focal cerebral ischemia. Exp. Neurol. 264, 1-7.

Yan-Chun, L., Hong-Mei, Y., Zhi-Hong, C., Qing, H., Yan-Hong, Z., Ji-Fang, W., 2017. MicroRNA-192-5p Promote the Proliferation and Metastasis of Hepatocellular Carcinoma Cell by Targeting SEMA3A. Appl. Immunohistochem. Mol. Morphol. $25,251-260$.

Ye, W., Lv, Q., Wong, C.-K.A., Hu, S., Fu, C., Hua, Z., Cai, G., Li, G., Yang, B.B., Zhang, Y., 2008. The effect of central loops in miRNA:MRE duplexes on the efficiency of miRNA-mediated gene regulation. PLoS One 3, e1719.

Yi, R., Qin, Y., Macara, I.G., Cullen, B.R., 2003. Exportin-5 mediates the nuclear export of pre-microRNAs and short hairpin RNAs. Genes Dev. 17, 3011-6.

Yu, D., dos Santos, C.O., Zhao, G., Jiang, J., Amigo, J.D., Khandros, E., Dore, L.C., Yao, Y., D’Souza, J., Zhang, Z., Ghaffari, S., Choi, J., Friend, S., Tong, W., Orange, J.S., Paw, B.H., Weiss, M.J., 2010. miR-451 protects against erythroid oxidant stress by repressing 14-3-3zeta. Genes Dev. 24, 1620-33.

Zhang, J., Storey, K.B., 2016. RBioplot: an easy-to-use R pipeline for automated statistical analysis and data visualization in molecular biology and biochemistry. PeerJ 4, e2436. 


\section{CHAPTER 4}

\section{Frozen Liver MicroRNAs}




\section{Biogenesis and regulation of the freeze-thaw responsive microRNA fingerprint in wood frog liver}

Hanane Hadj-Moussa and Kenneth B. Storey*

Department of Biology and Institute of Biochemistry, Carleton University, 1125 Colonel By Drive, Ottawa, Ontario K1S 5B6, Canada

*Corresponding author: kenneth.storey@carleton.ca 


\section{ABSTRACT}

Freeze tolerant animals undergo significant physiological and biochemical changes to overcome the challenges associated with prolonged whole body freezing. An extreme example of this phenomena is the freeze tolerant wood frog, Rana sylvatica, that freezes up to $65 \%$ of total body water as extracellular ice masses. When the frog retreats into this state of suspended animation, it displays no detectable brain activity, no movement, no breathing, and a flat-lined heart. To survive extensive freezing, it integrates various regulatory mechanisms to ensure quick and smooth transitions into this hypometabolic state. One such rapid and reversible regulatory molecule capable of coordinating all aspects of biological functions is microRNA. Herein, we present a large-scale analysis of the biogenesis and regulation of microRNAs in wood frog liver over the course of a freezethaw cycle (control, $24 \mathrm{~h}$ frozen, and $8 \mathrm{~h}$ thawed). Immunoblotting of key microRNA biogenesis factors showed an upregulation and enhancement of microRNA processing capacity during freezing and thawing. This was followed with RT-qPCR analysis of 109 microRNA species; of which 20 were significantly differentially expressed during freezing and thawing, with the majority displaying a general pattern of upregulation. Downstream bioinformatics miRNA-mRNA targeting coupled with, protein-protein interactions, and functional clustering of biological processes suggests these microRNAs are suppressing pro-growth functions including; DNA replication, mRNA processing and splicing, protein translation and turnover, and carbohydrate metabolism. Our findings suggest that the enhanced miRNA maturation capacity observed might be responsible for the vital hepatic miRNA-mediated suppression of energy-expensive processes needed for freeze survival.

KEYWORDS: Rana sylvatica; freeze tolerance; miRNA; metabolic rate depression; FINDTAR3 


\subsection{INTRODUCTION}

Animals have evolved various adaptations to overcome the challenges associated with extreme winter landscapes that include; low temperatures, food scarcity, short photoperiods, etc. One key theme employed in the various winter survival strategies that range from hibernation, to freeze avoidance, to freeze tolerance, is the use of hypometabolism (Storey and Storey, 2004). Depressing metabolic rate to less than $30 \%$ of active levels allows these animals to survive prolonged periods of dormancy using only internal fuel reserves. This requires extensive molecular reorganization and energy reprioritization to processes necessary for vital cellular functions and pro-survival pathways (Storey and Storey, 2017). One of the more extreme survival strategies is freeze tolerance, and an excellent model of natural vertebrate freeze tolerance is the North American wood frog Rana sylvatica (also known as Lithobates sylvaticus). The wood frog, that ranges from the Southern Appalachians to Alaska, can retreat into a frozen state of suspended animation, for months at a time, and can endure the freezing of up to $65 \%$ of total body water as extracellular ice masses (Lee-Yaw et al., 2008). When frozen, wood frogs display no detectable brain activity, no breathing, no movement, and a flat-lined heart; and yet, when temperatures warm they are able to thaw, unscathed. Various adaptations are in place to facilitate successful freeze tolerance, these include; [1] minimization of cell volume reduction and excessive extracellular ice formation via the synthesis and distribution of cryoprotectant glucose (Storey, 1990), [2] global metabolic rate depression and reprioritization of energy usage, and [3] upregulation and activation of select pro-survival and protective mechanisms (Storey and Storey, 2017). Indeed, despite being in a hypometabolic state, wood frogs must expend energy on protective functions, 
such as antioxidant defenses (Dawson and Storey, 2016), to ensure that they can endure the myriad of freeze-associated challenges that include; dehydration, mechanical damage from ice, anoxia/ischemia, hyperglycemia, etc. (Storey and Storey, 2017).

Numerous molecular mechanisms are in place to orchestrate smooth transitions between active and frozen dormant states. These intricate regulatory mechanisms, that have been reported in various hypometabolic strategies, include; epigenetic regulation through DNA and histone modifications, signal transduction pathways, regulation by transcription factors, regulatory post-translational modifications, and post-transcriptional regulation by RNA-binding proteins and microRNA (miRNA) expression (Storey, 2015). MicroRNAs are a key class of multifunctional molecules that are emerging as central modulators of diverse biological function, including those critical for environmental stress responses (Biggar and Storey, 2011). These highly conserved small non-coding RNA transcripts ( 22 nt) can selectively fine tune gene expression of essential and non-essential cellular functions by binding to over $60 \%$ of protein-coding genes in humans, and either promote their degradation or induce their translation suppression (Davis-Dusenbery and Hata, 2010; Ebert and Sharp, 2012). MicroRNA-mediated gene silencing is a prime regulatory candidate that can promote the reorganization required to facilitate the global metabolic rate depression and selective gene activation that is characteristic of hypometabolic stress responses. The inherent properties that make miRNAs excellent regulators include their capacity to be; [1] rapidly activated, [2] readily reversible, [3] easily inducible, [4] energetically inexpensive, and [5] responsive to environmental stimuli (Storey, 2015). The regulatory capacity of miRNAs is further enhanced by the ability of a single miRNA to 
target hundreds of mRNAs and a single mRNA transcript to be targeted by hundreds of miRNAs (Lui et al., 2015).

As this study examined miRNAs in a freeze tolerant model, the influence that temperature has on dictating miRNA functionality must also be investigated. While the binding of miRNA to mRNA targets is mainly dictated by the degree of complementarity at the seed region, it should be noted that the 3' end of miRNAs have been shown to bind in a manner that stabilizes the miRNA-mRNA duplex (Biggar and Storey, 2014). Therefore, the thermodynamic threshold of $-18 \mathrm{kcal} / \mathrm{mol}$ that is generally used to predict miRNA-mRNA targeting suggests that large fluctuations in body temperature will significantly affect miRNA targeting (Biggar and Storey, 2014; Wu et al., 2002). Indeed, at low temperatures, more miRNA-mRNA interactions will be stabilized which in turn increases the dynamic regulatory potential of these molecules. This has been demonstrated in a pilot study on freeze tolerant turtles that reported significant changes and enhanced miRNA functionality and targeting in frozen states (Biggar and Storey, 2015). An additional temperaturedependant effect concerns the thermodynamic stability of processed miRNA duplexes, in which even small shifts in temperature have been shown to modify strand-specific expression of miRNAs (Khvorova et al., 2003; Potla et al., 2015). Taken together, the large number of miRNA species, their complementary mRNA targets, and the temperaturedependant effects on binding all act to generate an expansive regulatory RNA network capable of enabling broad and flexible control over mRNA expression during stress.

MicroRNA biogenesis is an evolutionarily conserved stepwise process that has recently been shown to be under the tight spatial and temporal control of various regulatory factors (Davis-Dusenbery and Hata, 2010). The majority of miRNAs are biosynthesized via the 
canonical biogenesis pathway that begins with the transcription of long primary-miRNA (pri-miRNA) by RNA polymerase II. Pri-miRNA is then cleaved into a $\sim 70$ nt long RNA hairpin structure known as a precursor-miRNA (pre-miRNA), this is accomplished by the RNase III endonuclease DROSHA that is complexed with its binding partner DGCR8. Current processing models suggest that DGCR8 directs DROSHA to a specific cleavage site at the junction of the stem of the hairpin structure by recognizing the pri-miRNA ssRNA-dsRNA junction (Han et al., 2006). The pre-miRNA is then exported from the nucleus to the cytoplasm by the nuclear transporter EXPORTIN-5 (XPO5) and RAN-GTP, both of which act to facilitate export and to protect newly-formed pre-miRNA from nuclear degradation (Bohnsack et al., 2004). Processing is then continued by DICER, another member of the RNase III endonuclease class of enzymes, that cleaves the pre-miRNA transcript into a miRNA duplex composed of both the passenger strand, that is generally degraded, and the guide strand that functions as the mature miRNA (MacFarlane and Murphy, 2010). This is facilitated by the transactivator response RNA binding protein (TRBP) that complexes with DICER to monitor the length of mature miRNAs in addition to modulating the processing efficiency of select pre-miRNAs (Fukunaga et al., 2012; Lee and Doudna, 2012). A third elusive protein that is also present in this complex is protein kinase RNA activator (PACT), its role in miRNA maturation remains unknown (Fukunaga et al., 2012; Lee and Doudna, 2012). The mature miRNA strand then associates with the multi-component RNA-induced silencing complex (RISC) and a member of the Argonaute family of endonucleases (AGO 1-4) that then complementary bind to specific regions in the 3' untranslated region (3'-UTR) of target mRNA transcripts. Imperfect miRNA-mRNA binding leads to translational suppression via sequestration into stress granules and 
processing bodies (P-bodies), while perfect binding results in mRNA target degradation (Bartel, 2004).

As demonstrated by proton MRI, one of the last organs to succumb to ice accumulation and the first to thaw in wood frogs is the liver (Rubinsky et al., 1994). The liver is the metabolic centre of most organisms and is responsible for coordinating whole body metabolism, including; ketogenesis, gluconeogenesis and regulation of blood sugar levels, carbohydrate storage, detoxification, protein synthesis and release, and the processing and storage of nutrients. In addition to these vital processes, the liver takes on additional roles during freezing such as the synthesis and distribution of cryoprotectant glucose and freezeresponsive protein synthesis. This includes the enhanced synthesis of the blood clotting protein fibrinogen and novel freeze-responsive proteins FR-10 and FR-47 that function to ensure whole organism cryoprotection and freeze survival (Biggar et al., 2013; Cai and Storey, 1997; Sullivan et al., 2015). Within minutes of the initial ice nucleation event, the liver begins to convert its massive glycogen stores, that were accumulated during autumn (Storey and Storey, 1992), into glucose through the activation of select enzymes including glycogen phosphorylase. These newly-synthesized glucose molecules are immediately exported to the blood and the rest of the body to protect against excessive cellular dehydration and minimize intracellular and extracellular ice formation (Storey and Storey, 2017). While liver metabolic reorganization has been shown to be regulated by hormones, transcription factor networks, and enzymatic controls (Aguilar et al., 2017; Storey and Storey, 2017), recent work is now focusing on elucidating the role of post-transcriptional mechanisms in frozen wood frog livers. Initial wood frog miRNA studies performed on five hepatic miRNA species (miR-26a, miR-126, miR-217, miR-21, and miR-16), have 
shown these freeze-upregulated miRNAs to promote anti-apoptotic functions and cellcycle suppression (Biggar et al., 2009; Zhang and Storey, 2013). Furthermore, a more comprehensive miRNA examination in wood frog skeletal and cardiac muscles reported tissue-specific regulation of 53 miRNAs over the freeze-thaw cycle and suggested their involvement in maintaining muscle contractions and reversible protein phosphorylation (Bansal et al., 2016).

The rapidly expanding field of miRNAs has recently begun to examine their role in facilitating the adaptational strategies observed in nature that range from; estivating sea cucumbers (Chen et al., 2013), anoxia tolerant turtles and invertebrates (Biggar et al., 2012; Biggar and Storey, 2012), hibernating marsupials and squirrels (Hadj-Moussa et al., 2016; Liu et al., 2010), and even a few freeze tolerant and freeze avoidant animals and insects (Bansal et al., 2016; Lyons et al., 2013). In the present study, we performed a large-scale analysis of miRNA biogenesis and regulation in wood frog livers over the course of a freeze-thaw cycle. Immunoblotting of the key components of miRNA biogenesis machinery revealed a general upregulation during both freezing and thawing. RT-qPCR of the relative expression levels of 109 miRNAs revealed that the majority of differentially expressed miRNAs were upregulated during stress. This was further dissected by in silico miRNA target prediction and functional enrichment at the physiologically relevant low temperatures of $24 \mathrm{~h}$ frozen $\left(-2.5^{\circ} \mathrm{C}\right)$ and $8 \mathrm{~h}\left(5^{\circ} \mathrm{C}\right)$ thawed frogs. Characterization of the predicted downstream pathways identified a miRNA-mediated broad suppression of energy-expensive processes such as; DNA replication, protein translation and turnover, carbohydrate metabolism, etc. Taken together, our findings suggest that the increased miRNA maturation and processing capacity observed is responsible for the selective 
silencing of these - pathways thereby identifying a novel miRNA-mediated mechanism for the regulation and facilitation of successful wood frog freeze tolerance.

\subsection{MATERIALS AND METHODS}

\subsubsection{Animal experiments}

Male wood frogs were collected from spring meltwater ponds in Oxford Mills, Ontario, Canada. Frogs were washed in a tetracycline bath and acclimated at $5^{\circ} \mathrm{C}$ for two weeks in plastic containers lined with sphagnum moss. Active (non-stressed) control frogs were sampled from this condition. For the $24 \mathrm{~h}$ frozen condition, frogs were moved to $-2.5^{\circ} \mathrm{C}$ plastic containers lined with damp paper towel and the $24 \mathrm{~h}$ freezing exposure was timed from that point. Frozen frogs were randomly sampled from this condition. Remaining frogs were assigned to the recovery group and transferred to $5^{\circ} \mathrm{C}$ where they thawed for $8 \mathrm{~h}$. All control, $24 \mathrm{~h}$ frozen, and $8 \mathrm{~h}$ thawed frogs were euthanized by spinal pithing. Liver was rapidly excised, flash frozen in liquid nitrogen, and stored at $-80^{\circ} \mathrm{C}$. Animal care protocols and experimentation protocols were performed in accordance with the Carleton University Animal Care Committee and followed the guidelines set by the Canadian Council on Animal Care.

\subsubsection{Total soluble protein extraction}

Total soluble protein extracts were prepared from liver of control, $24 \mathrm{~h}$ frozen, $8 \mathrm{~h}$ thawed wood frogs. Samples $(\sim 500 \mathrm{mg})$ of frozen tissue were homogenized 1:2 w:v using a Polytron PT10 homogenizer and chilled homogenization buffer (20 mM HEPES, $200 \mathrm{mM}$ 
$\mathrm{NaCl}, 0.1 \mathrm{mM}$ EDTA, $10 \mathrm{mM} \mathrm{NaF}, 1 \mathrm{mM} \mathrm{Na} \mathrm{VO}_{4}, 10 \mathrm{mM} \beta$-glycerophosphate, $\mathrm{pH}$ 7.4) with a few crystals of PMSF and $1 \mu \mathrm{L} / \mathrm{mL}$ of protease inhibitor (Bioshop; Cat\# PIC002, ON, Canada). Homogenates were centrifuged for $15 \mathrm{~min}$ at $10,000 \mathrm{~g}\left(4^{\circ} \mathrm{C}\right)$, and soluble protein-containing supernatants were collected. The total protein concentration of each sample was quantified using the BioRad protein assay, as per the manufacturer's instructions (Cat\# 5000002; CA, USA). Protein concentrations were standardized to 10 $\mu \mathrm{g} / \mu \mathrm{L}$ using homogenization buffer. Standardized samples were mixed 1:1 $\mathrm{v} / \mathrm{v}$ with SDS buffer $(100 \mathrm{mM}$ Tris-HCl, 4\% w/v SDS, $20 \% \mathrm{v} / \mathrm{v}$ glycerol, $0.2 \mathrm{w} / \mathrm{v}$ bromophenol, $10 \% \mathrm{v} / \mathrm{v}$ $\beta$-mercaptoethanol, $\mathrm{pH}$ 6.8), to a final concentration of $5 \mu \mathrm{g} / \mu \mathrm{L}$. Finally, samples were boiled for 10 min to denature and linearize all proteins and then stored at $-40^{\circ} \mathrm{C}$ until use.

\subsubsection{Immunoblotting}

Equal amounts of total protein homogenates (25-40 $\mu \mathrm{g}$ depending on target being probed) of control, $24 \mathrm{~h}$ frozen, and $8 \mathrm{~h}$ thawed were loaded on 6-15\% discontinuous SDS-PAGE. Gels were run on a BioRad Mini Protean III system at $180 \mathrm{~V}$ for $1-3 \mathrm{~h}$ at $4^{\circ} \mathrm{C}$ in running buffer (25 mM Tris-base, $190 \mathrm{mM}$ glycine, 0.1\% w/v SDS, [pH 7.6]). Resolved protein gels were then transferred to $0.45 \mu \mathrm{m}$ pore polyvinylidene difluoride membranes at 160 $\mathrm{mA}$ for $1.5-16 \mathrm{~h}$ in pre-chilled transfer buffer $(25 \mathrm{mM}$ Tris-base, $192 \mathrm{mM}$ glycine $10 \% \mathrm{v} / \mathrm{v}$ methanol, [pH 8.5]), at $4^{\circ} \mathrm{C}$. Transferred membranes were air-dried for $15 \mathrm{~min}$, reactivated in methanol for $5 \mathrm{~min}$ and incubated in $2-10 \% \mathrm{v} / \mathrm{v}$ skim milk in TBST $(20 \mathrm{mM}$ Tris-base, $140 \mathrm{mM} \mathrm{NaCl}, 0.05 \% \mathrm{v} / \mathrm{v}$ Tween-20) for 10-45 min with rocking at room temperature (RT) (Chan et al., 2009). Blocked membranes were then washed 3 x 5 min in TBST and subsequently incubated overnight with the primary antibody of interest (1:1000 v:v dilution in TBST) with rocking at $4^{\circ} \mathrm{C}$. Primary antibodies were purchased for DROSHA 
(NeoBiolab; Cat\# A8336), DGCR8 (GeneTex; Cat\# GTX130061), DICER (SantaCruz; Cat\# SC-30226), AGO1 (GeneTex; Cat\# GTX47799), AGO2 (ECM BioSciences; Cat\# AP5281), p-AGO2 ${ }^{\text {Tyr393 }}$ (ECM BioSciences; Cat\# AP5311), p-AGO2 ${ }^{\mathrm{Ser} 387}$ (ECM BioSciences; Cat\# Ap5291), RAN (GeneTex; Cat\# GTX114139), EXPORTIN-5 (GeneTex; Cat\# 130727), TRBP (GeneTex; Cat\# GTX485546), and PACT (GeneTex; Cat\# GTX114215). Next, membranes were washed with TBST ( 3 x 5 min) and probed for 30 min at RT with horseradish peroxidase-conjugated goat anti-rabbit secondary antibody (1:8000 v/v dilution in TBST; BioShop; Cat\# APA002P). Finally, membranes were again washed $3 \times 5 \mathrm{~min}$ in TBST and protein bands were visualized using enhanced chemiluminescence $\left(\mathrm{H}_{2} \mathrm{O}_{2}\right.$ and Luminol $)$ and imaged using the ChemiGenius BioImaging System (Syngene, MD, USA). Total protein levels were visualized by staining membranes with Coomassie blue $(0.25 \% \mathrm{w} / \mathrm{v}$ Coomassie Brilliant Blue, $7.5 \% \mathrm{v} / \mathrm{v}$ acetic acid, $50 \%$ methanol) and subsequently destaining with destain solution $\left(50 \mathrm{ml} \mathrm{ddH}_{2} \mathrm{O}, 50 \mathrm{ml}\right.$ acetic acid, $150 \mathrm{ml}$ methanol).

\subsubsection{RNA isolation}

RNA isolation was performed on liver tissue ( $\sim 50 \mathrm{mg})$ of control, $24 \mathrm{~h}$ frozen, and $8 \mathrm{~h}$ thawed wood frogs ( $n=4$ individual animals) as previously described (Hadj-Moussa et al., 2016). Briefly, samples ( $\sim 50 \mathrm{mg})$ of frozen tissue were homogenized and extracted in a Trizol-choloroform extraction. RNA was precipitated with isopropanol and washed with $70 \%$ ethanol prior to being air-dried and resuspended in RNase-free water. RNA concentrations and purity were determined spectrophotometrically with the $260 / 280 \mathrm{~nm}$ ratio, using a Take3 micro-volume quantification plate (BioTek) and a PowerWave HT spectrophotometer (BioTek). Only samples with 260/280 ratios $>1.8$ were used for 
miRNA analyses. Total RNA integrity was then assessed by running gel electrophoresis on a $1 \%$ agarose gel stained with SYBR Green and by verifying the presence of sharp bands for $28 \mathrm{~S}$ and $18 \mathrm{~S}$ ribosomal RNA. RNA isolates were then standardized to a final concentration of $1 \mu \mathrm{g} / \mu \mathrm{L}$ with RNase-free water and stored at $-80^{\circ} \mathrm{C}$.

\subsubsection{Polyadenylation and stem-loop reverse transcription}

Extracted RNA samples were prepared for miRNA analysis following the protocol outlined by (Biggar et al., 2014). Polyadenylation was performed using the Epi-Bio PolyA tailing kit, where each $10 \mu \mathrm{L}$ reaction contained $3 \mu \mathrm{g}$ total RNA, $1 \mathrm{mM} \mathrm{ATP}$, and $0.5 \mu \mathrm{L}(2 \mathrm{U})$ of E. coli poly (A) polymerase in buffered solution $(0.1 \mathrm{M}$ Tris- $\mathrm{HCl}[\mathrm{pH} 8.0], 0.25 \mathrm{M} \mathrm{NaCl}$, and $10 \mathrm{mM} \mathrm{MgCl} 2$ ). Reactions were incubated at $37^{\circ} \mathrm{C}$ for $30 \mathrm{~min}$ to adenylate, $95^{\circ} \mathrm{C}$ for 5 min to arrest, and then immediately chilled on ice. The polyadenylated products $(10 \mu \mathrm{L}$ samples) were combined with $5 \mu \mathrm{L}$ of $250 \mathrm{pM}$ stem-loop RT adapter primer, heated to $95^{\circ} \mathrm{C}$ for $5 \mathrm{~min}$ to denature RNA, cooled to $60^{\circ} \mathrm{C}$ for $5 \mathrm{~min}$ to allow adapter annealing, and placed on ice for 1 min (Supp. Table S4.1). The SuperScript ${ }^{\mathrm{TM}}$ III Reverse Transcriptase kit was used on the polyadenylated and stem-loop primer-annealed RNA samples. Briefly, each sample was combined with; $1 \mu \mathrm{L}$ mouse Maloney leukemia virus (M-MLV) reverse transcriptase (2 $\mathrm{U}), 1 \mu \mathrm{L}$ deoxynucleotide triphosphate (dNTP) mixture containing $25 \mathrm{mM}$ of each nucleotide (ThermoFisher Scientific; Cat\# R1121), $2 \mu \mathrm{L} 0.1 \mathrm{M}$ dithiothreitol (DTT), and $4 \mu \mathrm{L} 5 \mathrm{x}$ first-strand buffer. Samples were incubated at $16^{\circ} \mathrm{C}$ for $30 \mathrm{~min}, 42^{\circ} \mathrm{C}$ for $30 \mathrm{~min}$, and $85^{\circ} \mathrm{C}$ for $5 \mathrm{~min}$. cDNA was serially diluted and frozen at $-20^{\circ} \mathrm{C}$. 


\subsubsection{Relative microRNA quantification}

MicroRNA-specific forward primers were designed using annotated Xenopus miRNA sequences obtained from miRBase (Release 21) and XenBase (accessed September 2016). All miRNA, universal reverse primer, and reference gene primers were designed as described by (Biggar et al., 2014) and are listed in (Supp. Table S4.1). Primers were synthesized by Integrated DNA Technologies. All RT-qPCR assays were performed as previously described (Pellissier et al., 2006) using a CFX Connect ${ }^{\mathrm{TM}}$ Real-Time PCR Detection System (BioRad; Cat\# 1855201), following MIQE guidelines (Bustin et al., $2009)$. Each $20 \mu \mathrm{L}$ RT-qPCR reaction consisted of $2 \mu \mathrm{L}$ of diluted RT product, $10.115 \mu \mathrm{L}$ of $\mathrm{ddH}_{2} \mathrm{O}, 4 \mu \mathrm{L}$ of $1 \mathrm{M}$ trehalose (BioShop; Cat\# TRE222), $2 \mu \mathrm{L}$ RT-qPCR buffer (100 $\mathrm{mM}$ Tris-HCl [pH 8.5], $500 \mathrm{mM} \mathrm{KCl,} \mathrm{1.5 \%} \mathrm{Triton} \mathrm{X-100,} 20 \mathrm{mM} \mathrm{MgCl}, 2$ mM dNTPs, and $100 \mathrm{nM}$ fluorescein), $0.5 \mu \mathrm{L}$ formamide (BioShop; Cat\# FOR001), $0.5 \mu \mathrm{L}$ of $25 \mathrm{mM}$ miRNA-specific forward primer, $0.5 \mu \mathrm{L}$ of $25 \mathrm{mM}$ universal reverse primer, $0.1 \mu \mathrm{L}$ of 100x SYBR green mix diluted in dimethyl sulfoxide (Invitrogen; Cat\# S7585), $0.16 \mu \mathrm{L}$ of $25 \mathrm{mM}$ dNTPs, and $0.125 \mu \mathrm{L}$ of $5 \mathrm{U} / \mu \mathrm{L}$ Taq polymerase (BioShop; Cat\# TAQ001.1). Reactions were incubated in a 96 -well plate at $95^{\circ} \mathrm{C}$ for $3 \mathrm{~min}$, followed by 40 cycles of $95^{\circ} \mathrm{C}$ for $15 \mathrm{~s}$, and $60^{\circ} \mathrm{C}$ for $1 \mathrm{~min}$. To ensure primer specificity and the amplification of a single PCR product, all RT-qPCR assays were subjected to post-run melt-curve analysis; reactions that generated non-specific products were rejected.

\subsubsection{Bioinformatics microRNA target identification and pathway enrichment}

To identify potential miRNA-mRNA interactions, mature miRNA sequences were searched against the 3'UTR sequences from the $X$. tropicalis reference genome available on the UCSC table browser (JGI 7.0/xenTro7) using the temperature sensitive miRNA 
target prediction program FINDTAR3 (v.3.11.12) (Ye et al., 2008). Target predictions were performed at $-2^{\circ} \mathrm{C}$ and $5^{\circ} \mathrm{C}$ for miRNAs that significantly changed during freezing and thawing, respectively. The following default parameters were used with FINDTAR3: AT and GC weight of 5, GT weight of 2, a gap opening penalty of -8 , a gap extension penalty of -2 , target duplex with maximum threshold free energy $-20 \mathrm{kcal} / \mathrm{mol}$, and demand strict 5' seed pairing.

The list of predicted miRNA-targets generated by FINDTAR3 was then functionally enriched to examine the connectivity of miRNA-targeted mRNA. This was done by mapping protein-protein interactions using STRING Xenopus tropicalis medium confidence interactions (Szklarczyk et al., 2015). Two groups were queried separately: [1] the 10 miRNAs upregulated in frozen livers, and [2] the 13 miRNAs upregulated in thawed livers. Protein interaction networks were then modelled using CYTOSCAPE software (Shannon et al., 2003) and clustered based on STRING combined scores using Markov Clustering (MCL) with the following default parameters; MCL granularity of 2 and edge weight cut-off of 0.5. Enrichment for biological processes was performed using the Gene Ontology (GO) annotations available for $X$. tropicalis using the PANTHER classification system (v.11.1). The enriched biological processes discussed and highlighted are statistically significant clusters, as determined by Bonferroni tests of $p<0.05$.

\subsubsection{Quantification and statistics}

Relative protein densitometric quantification was performed on chemiluminescent immunoblot protein bands using GeneTools Software on a ChemiGenius BioImaging System (Syngene, MD, USA). Immunoblot band intensity in each lane was standardized against a group of Coomassie blue-stained protein bands to correct for any minor variations 
in sample loading. Immunoblot data for each experimental condition are expressed as means ( \pm SEM), relative to control values, with $n=4$ samples from different animals. Statistical analysis was performed by one-way ANOVA and a Dunnett's post-hoc test $(p<$ 0.05 accepted as significant) using the RBIOPLOT statistics and graphing R package (Zhang and Storey, 2016).

MicroRNA relative expression levels were calculated using the comparative $\Delta \Delta \mathrm{Cq}$ method (Bustin et al., 2009). Raw Cq values were transformed to the $2^{-\mathrm{Cq}}$ form, such that the miRNA of interest could be normalized to the endogenous U6 snRNA reference gene. U6 snRNA was experimentally determined to be a suitable reference gene based on its stable expression in R. sylvatica liver under all experimental conditions tested, as described by (Schmittgen and Livak, 2008). Data are mean relative expression levels ( \pm SEM), relative to control values, where $n=4$ independent biological replicates from different animals at each sampling point. Statistically significant changes in miRNA relative expression were identified when a one-way ANOVAs and Dunnett's post-hoc test yielded a $* p<0.05$. Statistical analyses and histogram generation was performed using the RBIOPLOT statistics and graphing R package (Zhang and Storey, 2016).

\subsection{RESULTS:}

\subsubsection{Protein expression of miRNA biogenesis and processing machinery}

Immunoblotting was used to examine abundance levels, relative to control, of proteins involved in miRNA biogenesis in wood frog livers over a freeze-thaw cycle. Protein levels of both components of the microprocessor, DROSHA and DGCR8, were found to significantly increase to $1.890 \pm 0.106$ and $1.802 \pm 0.078$ of the control, respectively (Fig. 
4.1). This significant upregulation was sustained during thawing in both DROSHA and DGCR8 with levels increased to $1.894 \pm 0.192$ and $2.152 \pm 0.202$ of control, respectively (Fig. 4.1). Levels of nuclear export machinery XPO5 and RAN were significantly upregulated during freezing to $2.019 \pm 0.120$ and $2.046 \pm 0.190$ of control, respectively. This observed upregulation was sustained during thawing in both XPO5 and RAN with levels increasing to $1.522 \pm 0.163$ and $2.349 \pm 0.095$ of control, respectively (Fig. 4.1). Protein abundance levels of the RISC complex components, DICER, TRBP, and PACT all remained constant over the course of the freeze-thaw cycle (Fig. 4.1). AGO1 protein abundance remained constant during both freezing and thawing (Fig. 4.1). While total $\mathrm{AGO} 2$ protein levels were unchanged in freezing and thawing, protein levels of p$\mathrm{AGO} 2^{\mathrm{Ser} 387}$ significantly decreased during thawing to $0.464 \pm 0.124$ of control (Fig. 4.1). Levels of p-AGO2 $2^{\mathrm{Ty} 393}$ significantly decreased to $0.137 \pm 0.028$ and $0.276 \pm 0.023$ of control during freezing and thawing, respectively (Fig. 4.1).

\subsubsection{Differential miRNA expression over the freeze-thaw cycle}

A large-scale analysis of the expression of 109 miRNA species using RT-qPCR identified 20 freeze-thaw responsive miRNAs in wood frog livers (Fig. 4.2). The majority of differentially expressed miRNAs revealed an overall pattern of upregulation, during both freezing and thawing. Of the 13 miRNAs found to display significant differential expression during freezing, the following 10 increased to $1.48-2.57$, relative to control; rsy-miR-101a-3p, rsy-miR-181a-3p, rsy-miR-192-5p, rsy-miR-199a-5p, rsy-miR-200a-3p, $r s y-m i R-210-3 p, r s y-m i R-221-3 p, r s y-m i R-222-3 p, r s y-m i R-22-3 p$, and $r s y-m i R-301-3 p$

(Fig. 4.2 and Supp. Table S4.2). The following three miRNAs were found to be 
significantly downregulated to $0.42-0.63$ of control levels during freezing; rsy-miR-93a5p, rsy-miR-9406-3p, and rsy-miR-9-5p (Fig. 4.2 and Supp. Table S4.2).

During thawing, 15 miRNAs were found to be significantly differentially expressed and similar to freezing, the majority of miRNAs were upregulated (Fig. 4.2). Of the 15 thawresponsive miRNAs, the following 13 were significantly upregulated during freezing to $1.432-3.481$ of control levels; rsy-miR-10a-5p, rsy-miR-145-5p, rsy-miR-181a-3p, rsymiR-192-5p, rsy-miR-200a-3p, rsy-miR-208-3p, rsy-miR-210-3p, rsy-miR-221-3p, rsy$m i R-23-3 p$, rsy-miR-27-3p, rsy-miR-30a-3p, and rsy-miR-429-3p (Fig. 4.2 and Supp. Table S4.2). Only $r s y-m i R-93 a-5 p$ and $r s y-m i R-9406-3 p$ were found to decrease to 0.426 \pm 0.069 and $0.592 \pm 0.085$ of control levels, respectively (Fig. 4.2 and Supp. Table S4.2). The following subset of six miRNAs was significantly elevated during both freezing and thawing; rsy-miR-181a-3p, rsy-miR-192-5p, rsy-miR-199a-5p, rsy-miR-200a-3op, rsy$m i R-210-3 p$, and $r s y-m i R-221-3 p$. Conversely, $r s y-m i R-93 a-5 p$ and $r s y-m i R-9406-3 p$ were downregulated during both freezing and thawing (Fig. 4.2 and Supp. Table S4.2).

\subsubsection{Bioinformatic analyses of miRNA-targeted pathways}

The functional cluster enrichment of downstream miRNA targets was performed using temperature-sensitive miRNA-mRNA target prediction, protein-protein interaction analysis, and followed by the identification of enriched biological processes. The key processes found to be targeted by the 10 freeze-upregulated miRNAs were as follows; [1] intracellular signal transduction, [2] RNA processing and splicing, [3] protein ubiquitination, [4] carbohydrate catabolic processes and hexose metabolism, [5] DNA replication, [6] translation pre-initiation complex formation, [7] protein de-ubiquitination, [8] cell redox homeostasis, [9] ATP hydrolysis, [10] microtubule nucleation and 
polymerization, [11] intraciliary transport, and [12] lipid phosphorylation (Fig. 4.3 and

Supp. Table S4.3). The key networks targeted by the subset of 13 thaw-upregulated miRNAs included the following processes; [1] chromatin remodelling, [2] protein ubiquitination, [3] RNA processing and splicing, [4] carbohydrate catabolism and hexose metabolism, [5] tissue development, [6] microtubule nucleation and polymerization, [7] translation pre-initiation complex formation, [8] ATP hydrolysis, [9] intraciliary transport, [10] lipid phosphorylation, [11] DNA replication (Fig. 4.4 and Supp. Table S4.4). Due to the current limitations of the annotated $X$. tropicalis protein network interactions, the majority of remaining clusters were unclassified or mapped to non-liver specific processes.

\subsection{DISCUSSION}

The enormous regulatory potential of miRNAs that allows them broad reach into all aspects of the cellular landscape renders them excellent regulators of environmental stress responses. The role miRNAs play in facilitating cold-tolerance appears to be present in both vertebrates and invertebrates with a subset of "CryomiRs", cold-associated miRNAs, being implicated in biological processes involved in muscle atrophy prevention, cell cycle regulation, and glucose and lipid metabolism (Lyons et al., 2013). While freeze tolerance is characterized by a global depression of metabolic rate, various cryoprotective mechanisms and tissue survival strategies are required to ensure the frog is able to thaw unscathed. The role miRNAs play in coordinating complex liver functions during periods of environmentally-induced hypometabolism has been reported in evolutionary distant species ranging from; hibernating South American marsupials and ground squirrels (HadjMoussa et al., 2016; Liu et al., 2010; Wu et al., 2016), estivating African clawed frogs (Wu 
et al., 2013), and hypoxic Tibetan pigs (Zhang et al., 2015). Indeed, wood frog livers must be under tight transcriptional and translational control to ensure that they are able to coordinate cryoprotectant glucose synthesis and distribution despite the backdrop of hypometabolism (Storey and Storey, 2017). As such, the main question we were interested in was, does the liver rely on miRNA-mediated silencing to regulate and suppress non-vital processes that are not required during prolonged periods of dormancy? The present study found that the protein levels of key miRNA biogenesis factors were upregulated during both freezing and thawing and that this was associated with an upregulation of the majority of the 20 miRNAs differentially expressed during freezing and thawing. This freeze-thaw specific miRNA fingerprint suggests that critical energy-expensive processes such as, DNA replication, protein synthesis and turnover, and carbohydrate metabolism must be "turned off" in the liver to facilitate successful whole-body freeze survival.

The tight regulation of miRNA biogenesis plays a large part in generating the unique miRNA expression fingerprint of different animals, tissues, developmental stages, cell types, sub-cellular compartments, and stress responses. Our investigation of the protein levels of key miRNA biogenesis factors suggested an enhanced capacity for miRNA biosynthesis (Fig. 4.1). The significantly elevated protein levels of the microprocessor complex subunits measured during freezing (Fig. 4.1) have been shown to promote miRNA processing (Jafari et al., 2013; Sperber et al., 2014). DROSHA has been implicated as the main regulator and rate-limiting step of miRNA biogenesis and developmental studies on Xenopus oocytes have demonstrated that increased DROSHA protein levels strongly boost miRNA biogenesis and pri-miRNA processing (Muggenhumer et al., 2014). The nuclear export of pre-miRNA to the cytoplasm for further processing also serves as a critical 
regulatory step in miRNA biogenesis, and increased levels of the karyopherin XPO5 and its regulator, the small GTPase RAN, observed during freezing suggests enhanced miRNA nuclear export (Fig. 4.1). Indeed, the overexpression of XPO5 has been shown to enhance RNA interference mediated by both short hairpin RNAs and by endogenous miRNAs (Köhler and Hurt, 2007; Yi et al., 2005). Once in the cytoplasm, the pre-miRNA is further cleaved by the endonuclease DICER, and its binding partners TRBP and PACT, to form a ds-miRNA duplex (Fukunaga et al., 2012). The unchanged levels of RISC proteins DICER, TRBP, and PACT, over the freeze-thaw cycle suggest that this critical step proceeds unaffected and a possible explanation could be that various other non-canonical miRNA biogenesis pathways such as, the DROSHA and DGCR8-independent pathway, spliceosome-dependent mechanisms, and the terminal uridylyl transferasedependent pathway, converge at Dicer processing and therefore levels of this global protein must remain constant (Fig. 4.1) (Ha and Kim, 2014). The constant protein levels of DICER observed herein match previous measurements of frozen wood frog liver DICER levels and demonstrate the consistency and reproducibility of these findings (Fig. 4.1) (Biggar et al., 2009).

The mature miRNA guide strand then binds to a member of the AGO protein family, typically AGO1 or AGO2, to induce the cleavage or translational suppression of target mRNAs. Interestingly, protein levels of both AGO1, that interacts with $\sim 30 \%$ of miRNAs, and $\mathrm{AGO} 2$, the sole $\mathrm{AGO}$ protein member with slicer activity that interacts and with $\sim 60 \%$ of miRNAs, remained constant over the freeze-thaw cycle (Fig. 4.1) (Wang et al., 2012). However, studies have shown that AGO2 functionality is heavily modulated by posttranslational modifications (PTM) and upon further examination, the significantly reduced 
levels of p-AGO2 $2^{\mathrm{Ty} 393}$ observed during freezing and thawing suggest an overall increase, or rather lack of inhibition, of AGO2 miRNA processing capacity (Fig. 4.1). Phosphorylation at Tyr-393 by EGFR has been shown to reduce AGO2 binding to DICER which in turn results in the inhibition of pre-miRNA processing and maturation and therefore suggests an increased miRNA processing ability (Shen et al., 2013). AGO2 is involved in both miRNA biogenesis and function and its phosphorylation at Ser-387 has been shown to lead to reduced mRNA cleavage and enhanced translational repression (Zeng et al., 2008). Indeed, the thaw-reduced levels of $A G O 2^{\text {Ser387 }}$ suggest that mRNA cleavage, as opposed to translation inhibition or destabilization, is favoured during thawing. This observation however requires a deeper investigation of miRNA translational silencing machinery and an analysis of stress granule and P-body components is required to confirm this hypothesis (Fig. 4.1). Overall, our examination of the protein and PTM levels of the main miRNA biogenesis factors suggests a potential activation of canonical miRNA biosynthesis during both freezing and thawing (Fig. 4.1). This enhanced capacity for miRNA biogenesis could be facilitating the selective increase of miRNAs identified to be overexpressed during freezing and thawing (Fig. 4.2).

Of the 109 miRNAs examined in this study, ten were significantly upregulated while only three were downregulated during freezing (Fig. 4.2). Accepted dogma states that elevated miRNA levels negatively regulate and therefore silence the genes under their control via translational suppression or degradation (Ha and Kim, 2014). Functional in silico target enrichment of the 10 freeze-upregulated miRNAs suggested the suppression of the following energy-expensive processes; RNA processing and splicing, DNA replication, intracellular signal transduction, microtubule nucleation and polymerization, protein 
turnover, and ATP hydrolysis (Fig. 4.3 and Supp. Table S4.3). The predicted suppression of these pathways was to be expected, as a well-known characteristic of freezing is the global suppression of ATP-demanding processes, such as cell growth and proliferation (Storey and Storey, 2017). A study on frozen wood frog liver has shown strong evidence of cell cycle suppression via reduced protein levels of Cyclin-dependant kinases (Cdks) and cyclins A and B1 (Zhang and Storey, 2012). This previously reported suppression of cell cycle elements could be mediated by the observed freeze-upregulated miRNAs predicted to target key components involved in the initiation of DNA replication and cell cycle progression. Targets include; Cdk 45, cyclin E2, Origin recognition complex subunits 1 and 6, DNA replication complex GINS3, and the transcription factor E2F4 that controls cell cycle progression and proliferation (Fig. 4.3 and Supp. Table S4.3).

One of the most energetically-expensive process, that utilizes $25-30 \%$ of total cellular energy production, is protein synthesis and turnover (Rolfe and Brown, 1997). It therefore makes metabolic sense that these pathways are predicted to be miRNA-silenced during freezing (Fig. 4.3). Specific miRNA targets that were identified include; translation preinitiation complex proteins, elongation initiation factors, poly-A binding protein, and 60S acidic ribosomal protein P0 (RPLP0). The predicted silencing of these mRNAs by the upregulated miRNAs would inhibit complex formation and potentially reduce overall protein synthesis (Fig. 4.3 and Supp. Table S4.3). Interestingly, a study on RPLP0 in wood frog liver showed a 2-fold freeze-upregulation of RPLP0 transcript levels but reported that RPLP0 protein levels remained unchanged. This disconnect between mRNA transcript and protein levels observed in this previous study could be a result of the targeting of $r s y-m i R-101 a-3 p$ to the RPLP0 mRNA transcript, as evident by the unchanged 
protein levels despite the increased mRNA levels (Wu and Storey, 2005) (Supp. Table S4.3). Furthermore, stress-responsive suppression of global protein synthesis and degradation is an essential strategy of hypometabolism that has been characterized in anoxia tolerance, estivation, hibernation, and freeze tolerance (Fraser et al., 2001; Frerichs et al., 1998; Hochachka et al., 1996; Storey and Storey, 2007, 2004). In addition to reducing protein translation, frozen frogs also need to maintain their existing protein pool and must therefore curb protein degradation. One possible miRNA-mediated mechanism for this is the predicted silencing of both protein ubiquitinating proteins (ubiquitin conjugating enzyme E2 and E3) and de-ubiquitinating proteins (ubiquitin specific proteases 1, 30, and 46) observed herein (Fig. 3 and Supp. Table S3).

As previously discussed, wood frog freeze survival is critically dependant on their ability to mobilize vast amounts of cryoprotectant glucose in liver via a glycogenolytic response that raises liver glucose levels from $5 \mathrm{mM}$ to greater $\sim 300 \mathrm{mM}$ within a few hours (Storey and Storey, 1986). To facilitate the synthesis of cryoprotectant glucose and its distribution to the rest of the body, in addition to maintaining high glucose levels within the liver itself when frozen, various routes of glucose catabolism must be inhibited. Interestingly, a subset of our freeze-upregulated miRNAs were predicted to do exactly that, by suppressing critical points of the carbohydrate metabolism and hexose catabolism pathways (Fig. 4.3). For example, the targeting of $r s y-m i R-199 a-5 p$ to aldolase B, an enzyme that catalyzes one of the major steps in the glycolytic pathway, suggests a novel miRNA-mediated mechanism for the suppression of the catabolism of hexose phosphates that is known to occur in frozen frogs (Supp. Table S4.3). Indeed, a study on the metabolic enzymes in wood frog livers showed aldolase activity to be significantly decreased during freezing 
(Cowan and Storey, 2001). Furthermore, suppression of other carbohydrate catabolism pathways such as the citric acid cycle is also required during freezing, and here we show that the freeze-upregulated $r s y-m i R-210-3 p$ was predicted to target NADP-isocitrate dehydrogenase, an enzyme that catalyzes the oxidative decarboxylation of isocitrate into $\alpha$-ketoglutarate (Supp. Table S4.3). In addition, the freeze-induced downregulation of $r s y$ miR-9-5p observed herein, is also implicated in promoting carbohydrate homeostasis and is potentially contributing to the elevated levels of gluconeogenesis needed for freezing (Fig. 4.2). This is likely being facilitated by FOXO1, as studies have shown that decreased levels of hepatic miR-9 expression activate gluconeogenesis and promote hepatic glucose production via a FOXO1-mechanism (Yan et al., 2016). Taken together, these newlyidentified hepatic miRNA modulation mechanisms help prevent the breakdown of valuable glucose cryoprotective stores and add an additional layer of regulatory complexity to the biochemical controls that are known to directly modulate these metabolic enzymes.

A major cell preservation strategy found to be targeted by the freeze-upregulated miRNAs was cellular redox (Fig. 4.3). Evidence from wood frogs, other hypometabolic animals, and oxygen deprived organisms have shown that the enhancement of antioxidant defenses is critical for protection against rapid oxygenation upon thawing and ischemia/reperfusion injury (Storey and Storey, 2017). It was therefore interesting to find that a subset of the freeze-upregulated miRNAs was predicted to suppress the mRNA transcripts of glutaredoxin 2, peroxiredoxin 1, and thioredoxin reductase (Supp. Table S4.3). These enzymes all act to boost antioxidant capacity of cells and promote the reduction of thioredoxin, an essential reactive oxygen species scavenging molecule (Kalinina et al., 2008). The incongruence observed between the prediction of miRNA-mediated 
suppression of this antioxidant process, that has been previously shown to be active, could potentially be due to the fact that many of the major antioxidant enzymes that have been examined in wood frogs were primarily found to be regulated at the post-translational level (Dawson and Storey, 2016; Storey and Storey, 2017). Indeed, a study on superoxide dismutase in frozen frogs found that while total protein levels of Cu-SOD and Mn-SOD were not upregulated during freezing, these enzymes were significantly post-translationally modified and displayed enhanced activity during freezing (Dawson et al., 2015). This observation that antioxidant enzymes might be primarily modulated at the posttranslational level suggests that the cell need not use its limited energy stores to synthesize new antioxidant enzymes and serves a possible energy-saving role that miRNAs may be coordinating.

Our examination of miRNA regulation after $8 \mathrm{~h}$ of thawing found 13 miRNAs to be upregulated and only 2 miRNAs to be downregulated. Downstream targeting analysis of the thaw-upregulated miRNAs revealed that there was sustained targeting between many of the ATP-demanding processes predicted to be suppressed during freezing (Fig. 4.3 and 4.4). While frogs that have been thawed for $8 \mathrm{~h}$ display a normal physiologically recovered phenotype, a full metabolic and molecular recovery is a slow process. Indeed, the degradation of glucose stores, the loss of anaerobic end products, and the restoration of ATP and phosphagen pools have been shown to take several days to return to control levels (Storey and Storey, 1986). This evolved delay in recovery is metabolically sensible as it facilitates survival over the course of the multiple freeze-thaw cycles wood frogs experience when overwintering and prevents them from prematurely depleting their limited energy reserves and from losing their ability to freeze. As such, our findings suggest that 
miRNAs are involved in maintaining this adaptive delay by sustaining the suppression of these energy-expensive processes $8 \mathrm{~h}$ into thawing.

An interesting thaw-specific miRNA target identified herein was the complex chromatin remodelling network (Fig. 4.4). To date, the role played by the main epigenetic mechanisms that elicit heritable changes to gene expression, being DNA and histone modifications, have been scarcely examined in freeze tolerant animals. A recent study on histone methylation in frozen wood frogs has begun to shed some light in this area with its report of a novel liver-specific pattern of histone methylation during freezing and thawing (Hawkins and Storey, 2017). One of the chromatin remodelling proteins predicted to be targeted by $r s y-m i R-208-3 p$, $r s y-m i R-30 a-3 p$, and $r s y-m i R-10 a-5 p$ was the SWI/SNFrelated matrix-associated actin-dependant regulator of chromatin subfamily B member 1 (SMARCB1). As SMARCB1 aides in relieving chromatin condensation and making DNA more accessible to transcriptional machinery, its silencing by the above miRNAs suggests that this relaxation of chromatin and enhanced transcription is not occurring during thawing (Stojanova and Penn, 2009). Another key chromatin remodelling protein identified as a target of the thaw-upregulated $r s y-m i R-192-5 p$ was the gene that encodes for DNA methyltransferase (DNMT) 1-associated protein 1 (DMAP1) (Supp. Table S4.4). This protein functions to repress transcription through its interaction with DNMT-1, a protein that methylates DNA resulting in silenced gene expression (Bogdanovi and Veenstra, 2009). It is therefore possible that miRNA-mediated suppression of DMAP1 could act to relieve the potential DNA methylation transcriptional repression that may have been in effect during freezing. Indeed, a recent study in an anoxia tolerant turtle, another model of extreme hypometabolism, found an overall increase in DNA methylation, and in the protein 
abundance and activity of DNMT enzymes in liver in response to prolonged anoxia exposures and suggested that this translates into the downregulation of gene expression during oxygen deprivation (Wijenayake and Storey, 2016). These contradictory effects of miRNAs on chromatin remodelling complexes demonstrates the complex and intricate regulation of epigenetic mechanisms and warrants further investigations into the gene targets of these chromatin remodelling complexes.

The overarching trend of miRNA-mediated adaptive metabolic rate depression identified in our study on the miRNA fingerprint of freezing and thawing appears to be evolutionarily conserved in the livers of distant organisms undergoing hypometabolic stress responses. A pioneering initial miRNA study on miR-16 and miR-21 in wood frog liver showed that levels of these two miRNAs were significantly increased in response to freezing and that they are likely playing a role in promoting anti-apoptotic functions and suppressing cell growth and cell cycle progression (Biggar et al., 2009). This finding supports our predicted targeting of DNA replication and growth pathways (Fig. 4.3). A small preliminary investigation of hepatic miRNAs in estivating Xenopus laevis showed miR-210 to be significantly upregulated during dehydration and suggested that this is linked to the hypoxic state Xenopus tissues experience during severe dehydration (Wu et al., 2013). This upregulation of miR-210 appears to be a conserved response in animals experiencing oxygen deprivation, as this was also observed in the hepatopancreas of the anoxic intertidal mollusc Littorina littorea and in our examination of frozen wood frog livers (Fig. 4.2) (Biggar et al., 2012; Wu et al., 2013). The overexpression of miR-210 has been shown to target iron-sulfur cluster assembly proteins during low oxygen availability, resulting in 
mitochondrial repression that has been shown to promote a shift to anaerobic metabolism via glycolysis to compensate for the reduced oxygen availability (Chan et al., 2009).

Furthermore, a comprehensive study on 117 miRNAs in the hibernating 13-lined ground squirrel (13LGS) found that hepatic miRNAs overexpressed during torpor targeted progrowth pathways such as, growth factor receptor signaling, regulation of glycolysis, and mitosis (Wu et al., 2016). Of particular interest is the overexpression of hepatic miR-181a levels in torpid 13LGS that leads to insulin resistance in hepatocytes and that has also been shown to be upregulated in patients and mouse models of Type 2 diabetes (Zhou et al., 2012). As hibernating ground squirrels are known to exhibit reversible insulin resistance over the torpor-arousal cycle, the overexpression of miR-181a could be one of the underlying mechanisms facilitating the stimulation of insulin resistance during the initial periods of hyperinsulinemia and could act to maintain storage of fats required to fuel hibernation (Buck et al., 2002; Florant et al., 1985). Coincidentally, $r s y-m i R-181 a$ was also overexpressed in frozen wood frog livers and is likely playing a role in instigating the insulin resistance required to accumulate cryoprotectant glucose and to survive freezing. While the pancreatic response of frozen frogs to $\sim 300 \mathrm{mM}$ rising glucose levels has been shown to be intact leading to the doubling of plasma insulin levels during freezing, the subsequent uptake of glucose into insulin-sensitive organs is not observed (Hemmings and Storey, 1994). Despite this feature being previously attributed to the novel sequence difference in wood frog insulin proteins that has been associated with low potency insulin, our findings point to a novel underlying regulatory miRNA mechanism that could also be contributing to the freeze-responsive insulin resistance required for freezing (Bajaj et al., 1986; Conlon et al., 1998). The global miRNA-mediated suppression of liver metabolism 
during hypometabolism was also observed in hibernating Arctic squirrels that were found to overexpress select miRNAs during torpor to suppress cell growth (Liu et al., 2010). However, this global response does not appear to be conserved in evolutionarily distant marsupials. Indeed, a study on the hibernating South American marsupial Dromiciops gliroides demonstrated an overall torpor-induced downregulation of differentially expressed hepatic miRNAs. This miRNA decrease was predicted to facilitate and potentially activate a liver-centered compensatory mechanism for thermoregulation evolved by the unique marsupial $D$. gliroides that has been shown to lack heat-generating brown adipose that is typically utilized by other mammals during hibernation (HadjMoussa et al., 2016). 


\subsection{CONCLUSION}

Overall, our findings highlight a new underlying miRNA-mediated mechanism that is in part responsible for facilitating the global metabolic suppression observed in wood frog livers over the course of the freeze-thaw cycle. The general freeze and thaw -induced miRNA upregulation observed herein may have been a result of the measured increase of the protein levels and enhanced capacity of the key miRNA biogenesis and processing factors. The miRNA fingerprint of frozen and thawed livers was predicted to silence key ATP-demanding functions that are involved in pro-growth processes and in maintaining cellular homeostasis, both of which are non-essential when animals are in a hypometabolic state. This pattern of miRNA-mediated silencing of energy-expensive processes during extreme stress responses appears to be evolutionarily conserved across distant species and survival strategies. This study emphasizes the complexity of the expansive miRNA networks required for successful freeze tolerance and highlights select miRNA species that could be further investigated as hepatic “on/off” switches with therapeutic potential.

\section{ACKNOWLEDGEMENTS}

This work was supported by a Discovery grant (Grant \# 6793) from the Natural Sciences and Engineering Research Council (NSERC) of Canada. KBS holds the Canada Research Chair in Molecular Physiology and HH holds an Ontario Graduate Student Scholarship.

\section{CONFLICT OF INTEREST}

The authors declare no conflict of interest. 

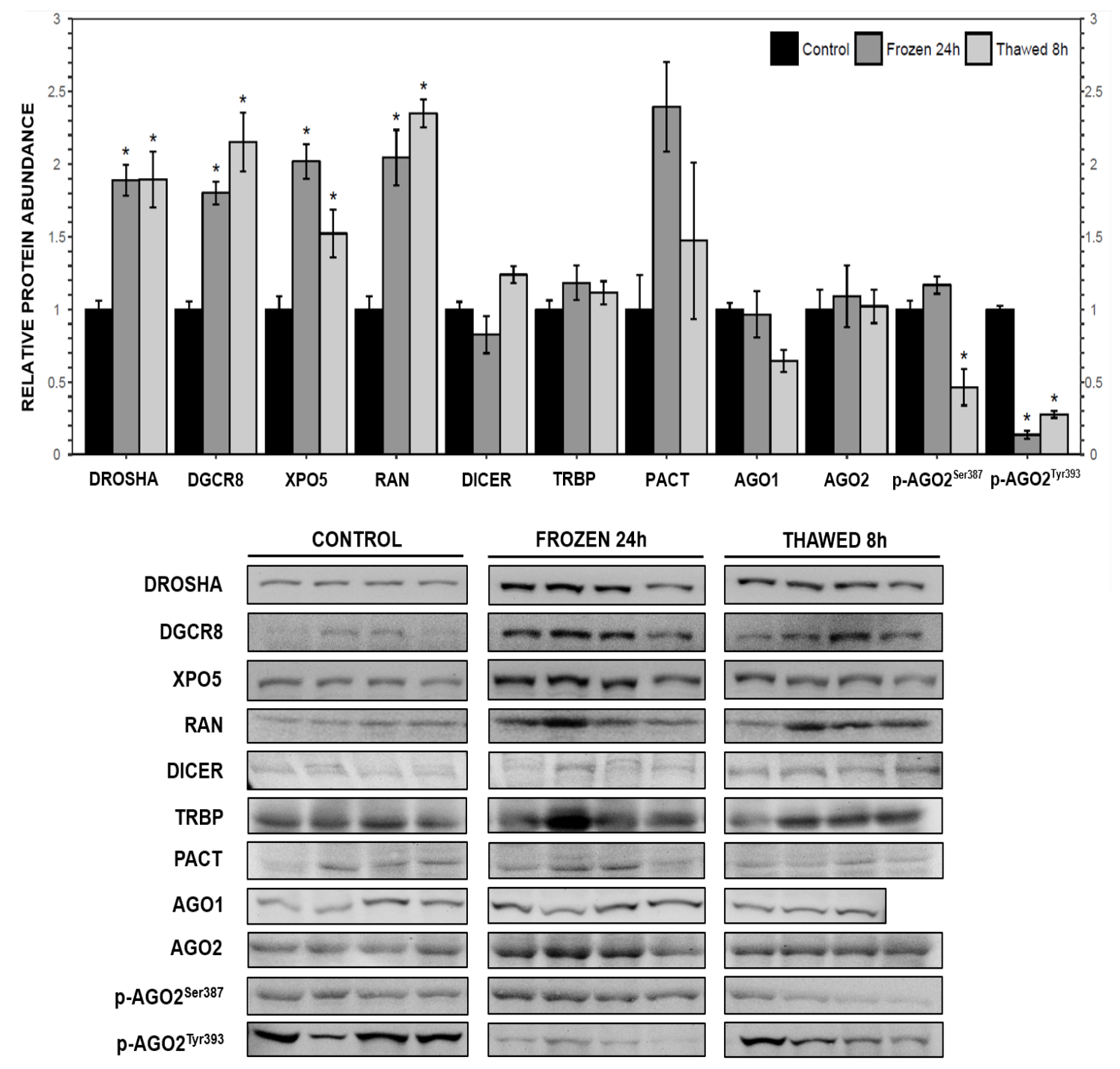

Figure 4.1. Analysis of miRNA biogenesis pathway protein levels in wood frog liver over a freeze-thaw cycle using immunoblotting. Upper panel displays histogram showing protein levels, relative to control, of DROSHA, DGCR8, XPO5, RAN, DICER, TRBP, PACT, AGO1, AGO2, p-AGO2 $2^{\mathrm{Ser} 387}$, and p-AGO2 $2^{\text {Tyr393 }}$ under control, $24 \mathrm{~h}$ frozen, and 8 $\mathrm{h}$ thawed conditions. Data are means \pm SEM of 3-4 independent biological replicates. Data are analyzed using a one-way ANOVA with a Dunnett's post hoc test, ${ }^{*} p<0.05$. Bottom panel show representative immunoblots of each protein and experimental condition. 


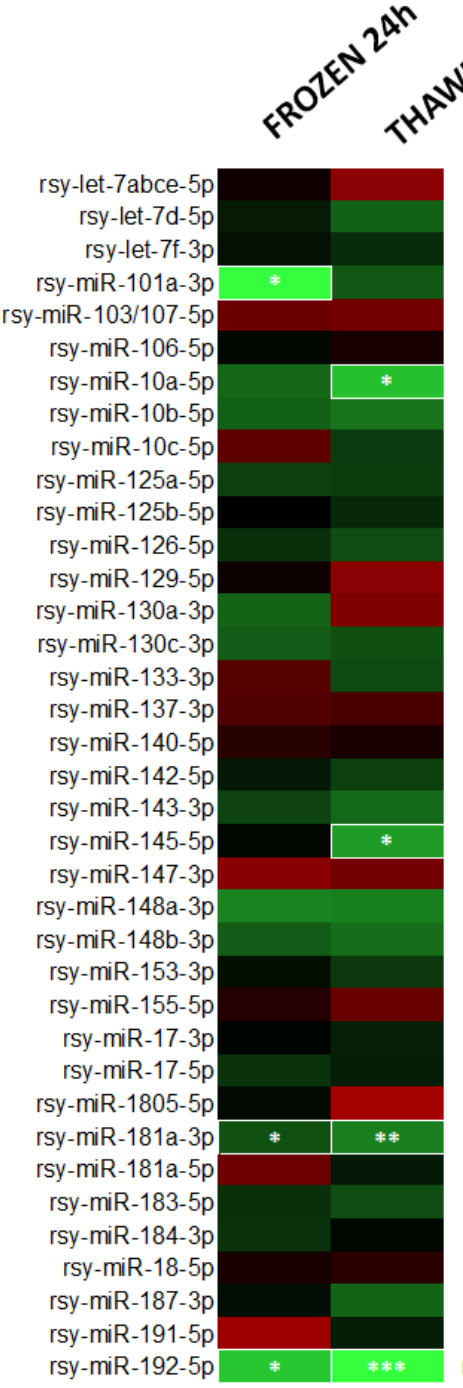

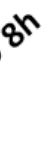

rsy-miR-193-3p rsy-miR-194-5p rsy-miR-196b-5p rsy-miR-199a-5p rsy-miR-199b-5p rsy-miR-19a-3p rsy-miR-19b-3p rsy-miR-1a-3p rsy-miR-200a-3p rsy-miR-200b-3p rsy-miR-202-3p rsy-miR-203-3p rsy-miR-204-5p rsy-miR-205-5p rsy-miR-206-3p rsy-miR-208-3p rsy-miR-20a-5p rsy-miR-20b-5p rsy-miR-210-3p rsy-miR-212-3p rsy-miR-214-3p rsy-miR-216-5p rsy-miR-2184-5p rsy-miR-2188-5p rsy-miR-221-3p rsy-miR-222-3p rsy-miR-223-3p rsy-miR-22-3p rsy-miR-22-5p rsy-miR-23-3p rsy-miR-24a-5p rsy-miR-24b-3p rsy-miR-25-3p rsy-miR-26-3p rsy-miR-27-3p rsy-miR-2970-5p rsy-miR-29abc-3p

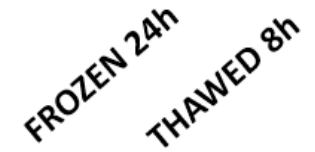

rsy-miR-29c-5p rsy-miR-29d-3p rsy-miR-301-3p rsy-miR-30a-3p rsy-miR-30a-5p rsy-miR-30b-5p rsy-miR-30d-5p rsy-miR-30e-5p rsy-miR-31b-5p rsy-miR-320-3p rsy-miR-338-3p rsy-miR-33a-5p rsy-miR-33b-5p rsy-miR-34a-5p rsy-miR-363-3p rsy-miR-365-3p rsy-miR-367-3p rsy-miR-425-5p rsy-miR-427-3p rsy-miR-429-3p rsy-miR-449a-5p rsy-miR-449b-5p rsy-miR-449c-3p rsy-miR-451-5p rsy-miR-455-5p rsy-miR-7-5p rsy-miR-92a-5p rsy-miR-92b-3p rsy-miR-93a-5p rsy-miR-9406-3p rsy-miR-9407-5p

rsy-miR-9-5p

rsy-miR-96-5p rsy-miR-98-5p rsy-miR-99/100-5p
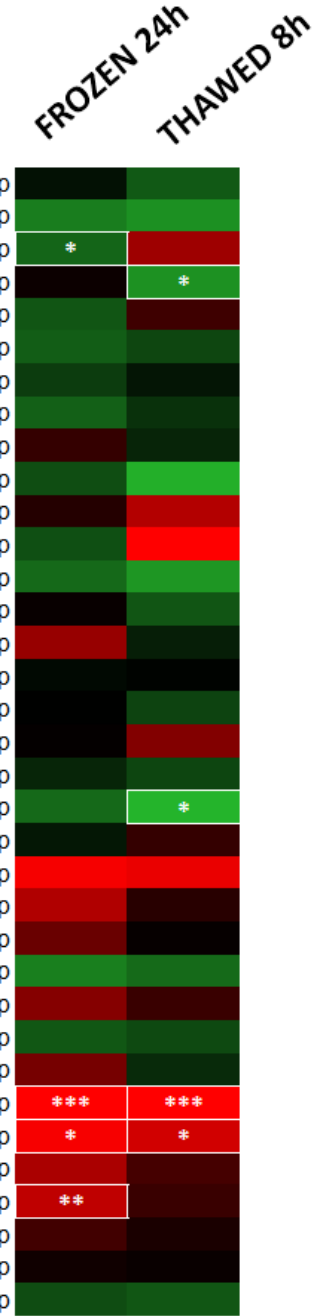

RELATIVE EXPRESSION $<0.5$ $>2.5$

Figure 4.2. Heatmap of RT-qPCR measured expression levels of 109 miRNA species examined in $24 \mathrm{~h}$ frozen and $8 \mathrm{~h}$ thawed wood frog livers, relative to control. All miRNAs were standardized against $U 6$ snRNA reference gene expression. Data are means \pm SEM of 3-4 independent biological replicates. Statistical testing used a one-way ANOVA with a Dunnett's post hoc test, $* p<0.05$. For the relative expression \pm SEM values of all 109 miRNA species examined refer to Supplementary Table S2. 
Figure 4.3. Functional target enrichment and network clustering of the subset of miRNAs upregulated in $24 \mathrm{~h}$ frozen wood frog livers. Downstream miRNA target prediction was performed at $-2^{\circ} \mathrm{C}$ using FINDTAR3. Protein-protein interactions of the downstream networks was performed using the STRING medium-confidence filter on the $X$. tropicalis database. MCL clustering and visualization was performed on CYTOSCAPE and coupled with GO ANALYSIS functional biological enrichment. [A] Full clustered protein-protein interaction map. [B] Representative set of significantly enriched proteinprotein interaction clusters. Refer to Supplementary Table S3 for more information on individual clusters, proteins, and targeting miRNA species. 


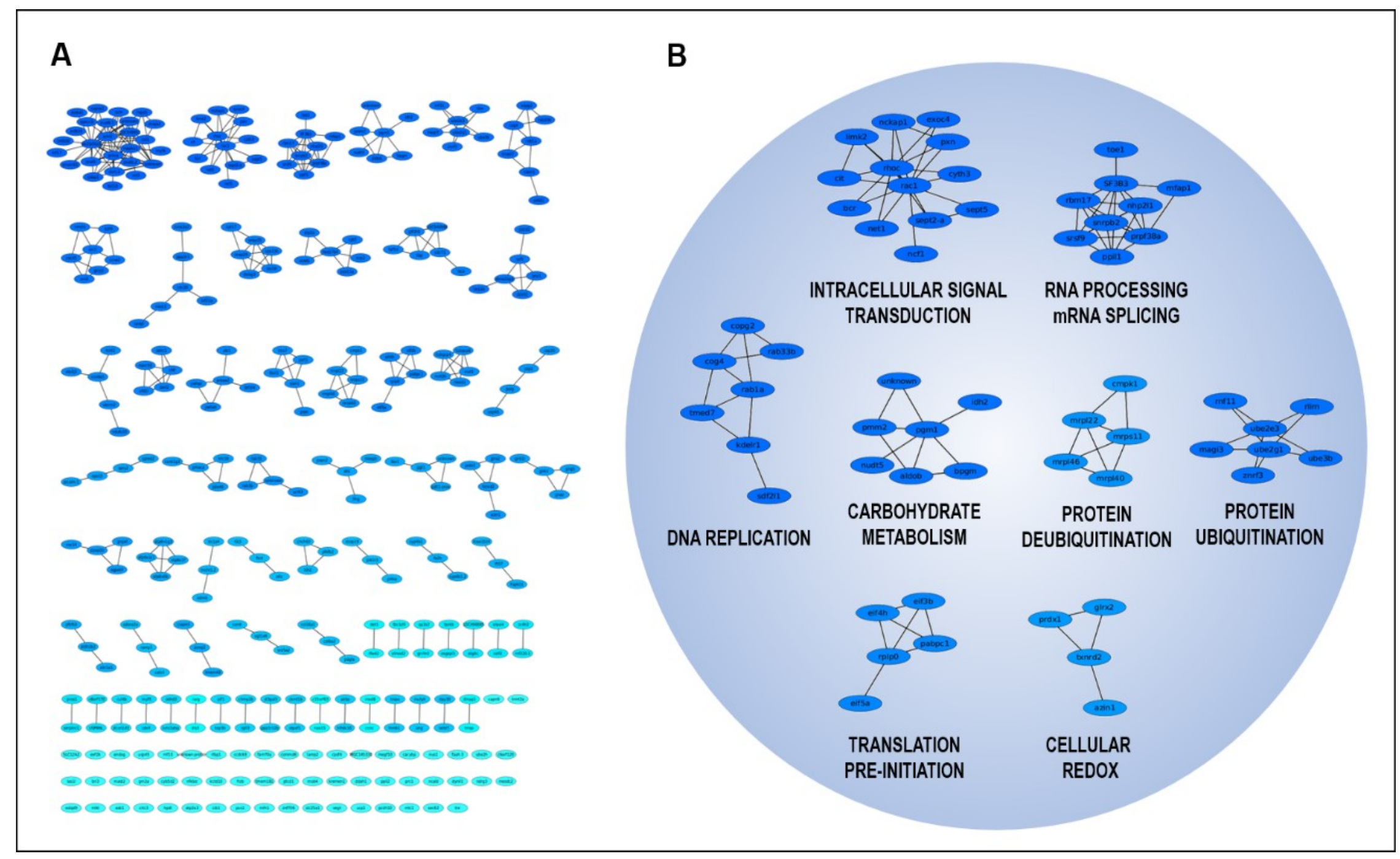


Figure 4.4. Functional target enrichment and network clustering of the subset of miRNAs upregulated in $8 \mathrm{~h}$ thawed wood frog livers. Downstream miRNA target prediction was performed at $5^{\circ} \mathrm{C}$ using FINDTAR3. Protein-protein interactions of the downstream networks was performed using the STRING medium-confidence filter on the $X$. tropicalis database. MCL clustering and visualization was performed on CYTOSCAPE and coupled with GO ANALYSIS functional biological enrichment. [A] Full clustered protein-protein interaction map. [B] Representative set of significantly enriched protein-protein interaction clusters. Refer to Supplementary Table S4 for more information on individual clusters, proteins, and targeting miRNA species. 


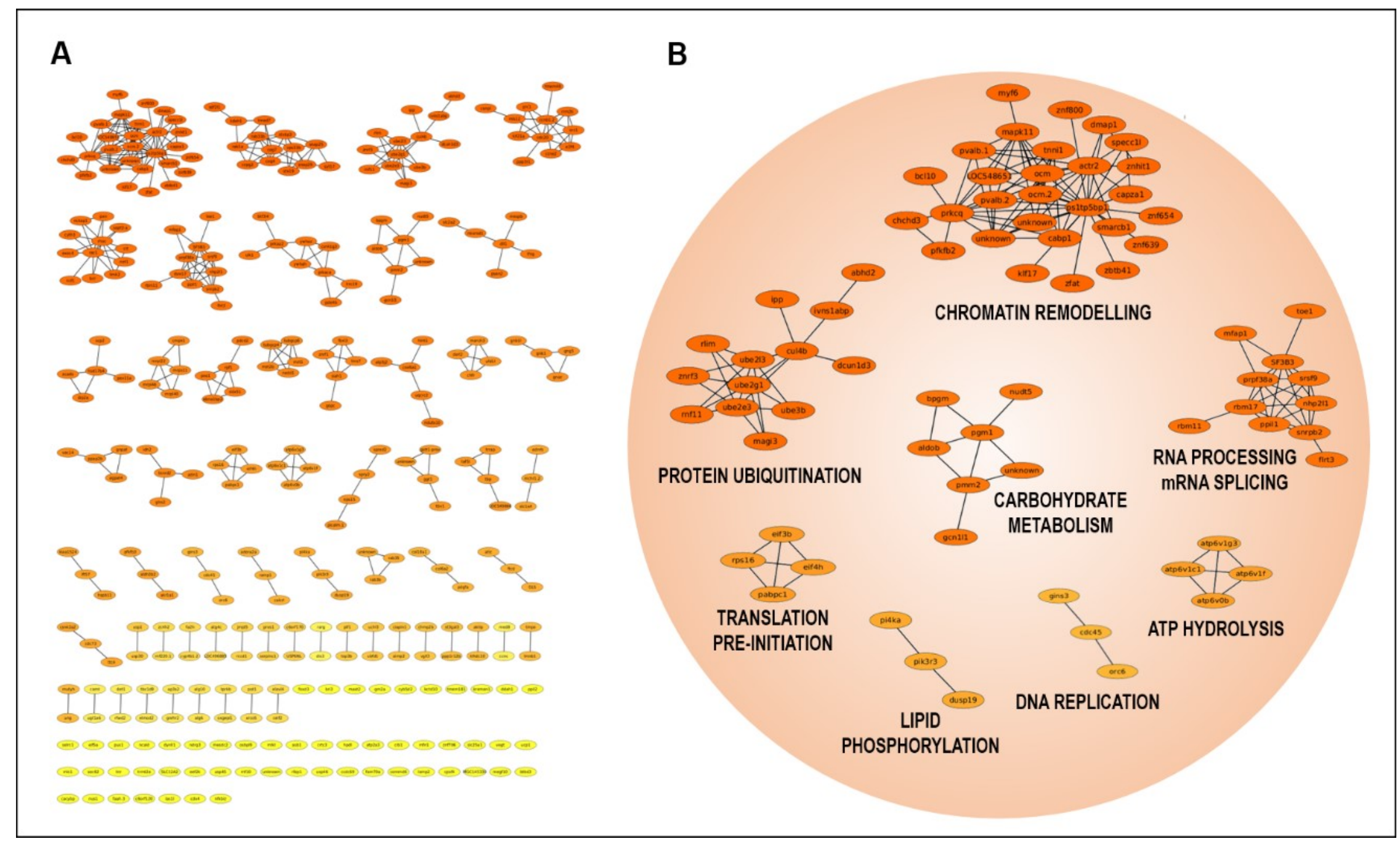




\section{SUPPLEMNTARY INFORMATION}

To access and download the four additional supplementary files that accompany this manuscript:

1. Visit: http://kenstoreylab.com/?page id=2899

2. Enter the password: HananeWantsToPass [* CaSe SeNsiTivE]

Supplementary Table S4.1. Primers used for analysis of miRNA expression in the liver of $R$. sylvatica, including miRNA-specific forward primers, universal reverse primer, and the stem-loop adapter for reverse-transcription.

Supplementary Table S4.2. Relative expression levels of 109 miRNA species examined in the liver of $R$. sylvatica. MicroRNA relative expression was evaluated by RT-qPCR of reverse-transcribed, polyadenylated transcripts. Data represent means of $n=3-4$ biological replicates from different animals \pm SEM. Relative expression of genes was calculated by standardizing against $U 6 s n R N A$ expression. Control values were adjusted to 1 and the $24 \mathrm{~h}$ frozen and $8 \mathrm{~h}$ thawed values were expressed relative to the control. Statistical testing used the Dunnett's test, values were considered significantly different from the corresponding control when $* p<0.05, * * p<0.01$, and $* * * p<0.001$.

Supplementary Table S4.3. Identity of the significantly enriched biological processes, protein members, and miRNA species identified using MCL clustered protein networks and functional GO ANALYSIS of the miRNAs upregulated in $24 \mathrm{~h}$ frozen wood frog liver.

Supplementary Table S4.4. Identity of the significantly enriched biological processes, protein members, and miRNA species identified using MCL clustered protein networks and functional GO ANALYSIS of the miRNAs upregulated in $8 \mathrm{~h}$ thawed wood frog liver. 


\section{REFERENCES [CHAPTER 4]}

Aguilar, O.A., Hadj-Moussa, H., Storey, K.B., 2017. Freeze-responsive regulation of MEF2 proteins and downstream gene networks in muscles of the wood frog, Rana sylvatica. J. Therm. Biol. 67, 1-8.

Bajaj, M., Blundell, T.L., Horuk, R., Pitts, J.E., Wood, S.P., Gowan, L.K., Schwabe, C., Wollmer, A., Gliemann, J., Gammeltoft, S., 1986. Соури insulin. Primary structure, conformation and biological properties of a hystricomorph rodent insulin. Biochem. J. 238, 345-51.

Bansal, S., Luu, B.E., Storey, K.B., 2016. MicroRNA regulation in heart and skeletal muscle over the freeze-thaw cycle in the freeze tolerant wood frog. J. Comp. Physiol. B. 186, 229-41.

Bartel, D.P., 2004. MicroRNAs genomics, biogenesis, mechanism, and function. Cell 116, 281-297.

Biggar, K.K., Dubuc, A., Storey, K., 2009. MicroRNA regulation below zero: Differential expression of miRNA-21 and miRNA-16 during freezing in wood frogs. Cryobiology $59,317-321$.

Biggar, K.K., Kornfeld, S.F., Maistrovski, Y., Storey, K.B., 2012. MicroRNA regulation in extreme environments: Differential expression of microRNAs in the intertidal snail Littorina littorea during extended periods of freezing and anoxia. Genomics. Proteomics Bioinformatics 10, 302-309.

Biggar, K.K., Kotani, E., Furusawa, T., Storey, K.B., 2013. Expression of freezeresponsive proteins, Fr10 and Li16, from freeze-tolerant frogs enhances freezing survival of BmN insect cells. FASEB J. 27, 3376-3383.

Biggar, K.K., Storey, K.B., 2015. Low-temperature microRNA expression in the painted turtle, Chrysemys picta during freezing stress. FEBS Lett. 589, 3665-3670.

Biggar, K.K., Storey, K.B., 2014. Insight into temperature-dependent microRNA function in mammalian hibernators. Temperature 1, 84-86.

Biggar, K.K., Storey, K.B., 2012. Evidence for cell cycle suppression and microRNA regulation of cyclin D1 during anoxia exposure in turtles. Cell Cycle 11, 1705-1713.

Biggar, K.K., Storey, K.B., 2011. The emerging roles of microRNAs in the molecular responses of metabolic rate depression. J. Mol. Cell Biol. 3, 167-175.

Biggar, K.K., Wu, C.-W., Storey, K.B., 2014. High-throughput amplification of mature microRNAs in uncharacterized animal models using polyadenylated RNA and stemloop reverse transcription polymerase chain reaction. Anal. Biochem. 462, 32-34.

Bogdanovi, O., Veenstra, G.J.C., 2009. DNA methylation and methyl-CpG binding proteins: developmental requirements and function. Chromosoma 118, 549-565.

Bohnsack, M.T., Czaplinski, K., Gorlich, D., 2004. Exportin 5 is a RanGTP-dependent dsRNA-binding protein that mediates nuclear export of pre-miRNAs. RNA 10, 185- 
91.

Buck, M.J., Squire, T.L., Andrews, M.T., 2002. Coordinate expression of the PDK4 gene: a means of regulating fuel selection in a hibernating mammal. Physiol. Genomics 8, $5-13$.

Bustin, S.A., Benes, V., Garson, J.A., Hellemans, J., Huggett, J., Kubista, M., Mueller, R., Nolan, T., Pfaffl, M.W., Shipley, G.L., Vandesompele, J., Wittwer, C.T., 2009. The MIQE guidelines: minimum information for publication of quantitative real-time PCR experiments. Clin. Chem. 55, 611-22.

Cai, Q., Storey, K.B., 1997. Freezing-induced genes in wood frog (Rana sylvatica): fibrinogen upregulation by freezing and dehydration. Am. J. Physiol. 272, R1480-92.

Chan, S.Y., Zhang, Y.-Y., Hemann, C., Mahoney, C.E., Zweier, J.L., Loscalzo, J., 2009. MicroRNA-210 controls mitochondrial metabolism during hypoxia by repressing the iron-sulfur cluster assembly proteins ISCU1/2. Cell Metab. 10, 273-284.

Chen, M., Zhang, X., Liu, J., Storey, K.B., Lee, D., 2013. High-throughput sequencing reveals differential expression of miRNAs in intestine from sea cucumber during aestivation. PLoS One 8, e76120. doi:10.1371/journal.pone.0076120

Conlon, J.M., Yano, K., Chartrel, N., Vaudry, H., Storey, K.B., 1998. Freeze tolerance in the wood frog Rana sylvatica is associated with unusual structural features in insulin but not in glucagon. J. Mol. Endocrinol. 21, 153-9.

Cowan, K.J., Storey, K.B., 2001. Freeze-thaw effects on metabolic enzymes in wood frog organs. Cryobiology 43, 32-45.

Davis-Dusenbery, B.N., Hata, A., 2010. Mechanisms of control of microRNA biogenesis. J. Biochem. 148, 381-392.

Dawson, N.J., Katzenback, B.A., Storey, K.B., 2015. Free-radical first responders: The characterization of $\mathrm{CuZnSOD}$ and MnSOD regulation during freezing of the freezetolerant North American wood frog, Rana sylvatica. Biochim. Biophys. Acta - Gen. Subj. 1850, 97-106.

Dawson, N.J., Storey, K.B., 2016. A hydrogen peroxide safety valve: The reversible phosphorylation of catalase from the freeze-tolerant North American wood frog, Rana sylvatica. Biochim. Biophys. Acta 1860, 476-85.

Ebert, M.S., Sharp, P.A., 2012. Roles for microRNAs in conferring robustness to biological processes. Cell 149, 515-24.

Florant, G.L., Lawrence, A.K., Williams, K., Bauman, W.A., 1985. Seasonal changes in pancreatic B-cell function in euthermic yellow-bellied marmots. Am. J. Physiol. 249, R159-65.

Fraser, K.P., Houlihan, D.F., Lutz, P.L., Leone-Kabler, S., Manuel, L., Brechin, J.G., 2001. Complete suppression of protein synthesis during anoxia with no post-anoxia protein synthesis debt in the red-eared slider turtle Trachemys scripta elegans. J. Exp. Biol. 204, 4353-60. 
Frerichs, K.U., Smith, C.B., Brenner, M., DeGracia, D.J., Krause, G.S., Marrone, L., Dever, T.E., Hallenbeck, J.M., 1998. Suppression of protein synthesis in brain during hibernation involves inhibition of protein initiation and elongation. Proc. Natl. Acad. Sci. U. S. A. 95, 14511-6.

Fukunaga, R., Han, B.W., Hung, J.-H., Xu, J., Weng, Z., Zamore, P.D., 2012. Dicer Partner Proteins Tune the Length of Mature miRNAs in Flies and Mammals. Cell 151, $533-$ 546.

Ha, M., Kim, V.N., 2014. Regulation of microRNA biogenesis. Nat. Rev. Mol. Cell Biol.

Hadj-Moussa, H., Moggridge, J.A., Luu, B.E., Quintero-Galvis, J.F., Gaitán-Espitia, J.D., Nespolo, R.F., Storey, K.B., 2016. The hibernating South American marsupial, Dromiciops gliroides, displays torpor-sensitive microRNA expression patterns. Sci. Rep. 6, 24627.

Han, J., Lee, Y., Yeom, K.-H., Nam, J.-W., Heo, I., Rhee, J.-K., Sohn, S.Y., Cho, Y., Zhang, B.-T., Kim, V.N., 2006. Molecular Basis for the Recognition of Primary microRNAs by the Drosha-DGCR8 Complex. Cell 125, 887-901.

Hawkins, L.J., Storey, K.B., 2017. Histone methylation in the freeze-tolerant wood frog (Rana sylvatica). J. Comp. Physiol. B.

Hemmings, S.J., Storey, K.B., 1994. Alterations in hepatic adrenergic receptor status in Rana sylvatica in response to freezing and thawing: implications to the freeze-induced glycemic response. Can. J. Physiol. Pharmacol. 72, 1552-60.

Hochachka, P.W., Buck, L.T., Doll, C.J., Land, S.C., 1996. Unifying theory of hypoxia tolerance: molecular/metabolic defense and rescue mechanisms for surviving oxygen lack. Proc. Natl. Acad. Sci. U. S. A. 93, 9493-8.

Jafari, N., Dogaheh, H.P., Bohlooli, S., Oyong, G.G., Shirzad, Z., Alibeiki, F., Asl, S.H., Zargar, S.J., 2013. Expression levels of microRNA machinery components Drosha, Dicer and DGCR8 in human (AGS, HepG2, and KEYSE-30) cancer cell lines. Int. J. Clin. Exp. Med. 6, 269-74.

Kalinina, E. V, Chernov, N.N., Saprin, A.N., 2008. Involvement of thio-, peroxi-, and glutaredoxins in cellular redox-dependent processes. Biochemistry. (Mosc). 73, $1493-510$.

Khvorova, A., Reynolds, A., Jayasena, S.D., 2003. Functional siRNAs and miRNAs exhibit strand bias. Cell 115, 209-16.

Köhler, A., Hurt, E., 2007. Exporting RNA from the nucleus to the cytoplasm. Nat. Rev. Mol. Cell Biol. 8, 761-773.

Lee-Yaw, J.A., Irwin, J.T., Green, D.M., 2008. Postglacial range expansion from northern refugia by the wood frog, Rana sylvatica. Mol. Ecol. 17, 867-884.

Lee, H.Y., Doudna, J.A., 2012. TRBP alters human precursor microRNA processing in vitro. RNA 18, 2012-2019. 
Liu, Y., Hu, W., Wang, H., Lu, M., Shao, C., Menzel, C., Yan, Z., Li, Y., Zhao, S., Khaitovich, P., Liu, M., Chen, W., Barnes, B.M., Yan, J., 2010. Genomic analysis of miRNAs in an extreme mammalian hibernator, the Arctic ground squirrel. Physiol. Genomics 42A, 39-51.

Lui, P.-Y., Jin, D.-Y., Stevenson, N.J., 2015. MicroRNA: master controllers of intracellular signaling pathways. Cell. Mol. Life Sci. 72, 3531-3542.

Lyons, P.J., Lang-Ouellette, D., Morin, P.J., 2013. CryomiRs: Towards the identification of a cold-associated family of microRNAs. Comp. Biochem. Physiol. Part D Genomics Proteomics 8, 358-364.

MacFarlane, L.-A., Murphy, P.R., 2010. MicroRNA: Biogenesis, Function and Role in Cancer. Curr. Genomics 11, 537-561.

Muggenhumer, D., Vesely, C., Nimpf, S., Tian, N., Yongfeng, J., Jantsch, M.F., 2014. Drosha protein levels are translationally regulated during Xenopus oocyte maturation. Mol. Biol. Cell 25, 2094-104.

Pellissier, F., Glogowski, C.M., Heinemann, S.F., Ballivet, M., Ossipow, V., 2006. Lab assembly of a low-cost, robust SYBR green buffer system for quantitative real-time polymerase chain reaction, Analytical Biochemistry.

Potla, R., Singh, I.S., Atamas, S.P., Hasday, J.D., 2015. Shifts in temperature within the physiologic range modify strand-specific expression of select human microRNAs. RNA 21, 1261-1273.

Rolfe, D.F., Brown, G.C., 1997. Cellular energy utilization and molecular origin of standard metabolic rate in mammals. Physiol. Rev. 77, 731-58.

Rubinsky, B., Wong, S.T., Hong, J.S., Gilbert, J., Roos, M., Storey, K.B., 1994. 1H magnetic resonance imaging of freezing and thawing in freeze-tolerant frogs. Am. J. Physiol. 266, R1771-7.

Schmittgen, T.D., Livak, K.J., 2008. Analyzing real-time PCR data by the comparative CT method. Nat. Protoc. 3, 1101-1108.

Shannon, P., Markiel, A., Ozier, O., Baliga, N.S., Wang, J.T., Ramage, D., Amin, N., Schwikowski, B., Ideker, T., 2003. Cytoscape: A software environment for integrated models of biomolecular interaction networks. Genome Res. 13, 2498-2504.

Shen, J., Xia, W., Khotskaya, Y.B., Huo, L., Nakanishi, K., Lim, S.-O., Du, Y., Wang, Y., Chang, W.-C., Chen, C.-H., Hsu, J.L., Wu, Y., Lam, Y.C., James, B.P., Liu, X., Liu, C.-G., Patel, D.J., Hung, M.-C., 2013. EGFR modulates microRNA maturation in response to hypoxia through phosphorylation of AGO2. Nature 497, 383-7.

Sperber, H., Beem, A., Shannon, S., Jones, R., Banik, P., Chen, Y., Ku, S., Varani, G., Yao, S., Ruohola-Baker, H., 2014. miRNA sensitivity to Drosha levels correlates with premiRNA secondary structure. RNA 20, 621-31.

Stojanova, A., Penn, L.Z., 2009. The role of INI1/hSNF5 in gene regulation and cancer. Biochem. Cell Biol. 87, 163-177. 
Storey, K.B., 2015. Regulation of hypometabolism: insights into epigenetic controls. J. Exp. Biol. 218, 150-159.

Storey, K.B., 1990. Life in a frozen state: adaptive strategies for natural freeze tolerance in amphibians and reptiles. Am. J. Physiol. 258, R559-68.

Storey, K.B., Storey, J.M., 2017. Molecular physiology of freeze tolerance in vertebrates. Physiol. Rev. 97, 623-665.

Storey, K.B., Storey, J.M., 2007. Tribute to P. L. Lutz: putting life on 'pause' - molecular regulation of hypometabolism. J. Exp. Biol. 210.

Storey, K.B., Storey, J.M., 2004. Metabolic rate depression in animals: transcriptional and translational controls. Biol. Rev. Camb. Philos. Soc. 79, 207-33.

Storey, K.B., Storey, J.M., 1992. Natural freeze tolerance in ectothermic vertebrates. Annu. Rev. Physiol. 54, 619-637.

Storey, K.B., Storey, J.M., 1986. Freeze tolerant frogs: cryoprotectants and tissue metabolism during freeze-thaw cycles. Can. J. Zool. 64, 49-56.

Sullivan, K.J., Biggar, K.K., Storey, K.B., 2015. Expression and characterization of the novel gene fr47 during freezing in the wood frog, Rana sylvatica. Biochem. Res. Int. 2015, 363912 .

Szklarczyk, D., Franceschini, A., Wyder, S., Forslund, K., Heller, D., Huerta-Cepas, J., Simonovic, M., Roth, A., Santos, A., Tsafou, K.P., Kuhn, M., Bork, P., Jensen, L.J., von Mering, C., 2015. STRING v10: protein-protein interaction networks, integrated over the tree of life. Nucleic Acids Res. 43, D447-D452.

Wang, D., Zhang, Z., O’Loughlin, E., Lee, T., Houel, S., O’Carroll, D., Tarakhovsky, A., Ahn, N.G., Yi, R., 2012. Quantitative functions of Argonaute proteins in mammalian development. Genes Dev. 26, 693-704.

Wijenayake, S., Storey, K.B., 2016. The role of DNA methylation during anoxia tolerance in a freshwater turtle (Trachemys scripta elegans). J. Comp. Physiol. B 186, 333-342.

Wu, C.-W., Biggar, K.K., Luu, B.E., Szereszewski, K.E., Storey, K.B., 2016. Analysis of microRNA expression during the torpor-arousal cycle of a mammalian hibernator, the 13-lined ground squirrel. Physiol. Genomics 48, 388-396.

Wu, C.W., Biggar, K.K., Storey, K.B., 2013. Dehydration mediated microRNA response in the African clawed frog Xenopus laevis. Gene 529, 269-275.

Wu, P., Nakano, S., Sugimoto, N., 2002. Temperature dependence of thermodynamic properties for DNA/DNA and RNA/DNA duplex formation. Eur. J. Biochem. 269, 2821-30.

Wu, S., Storey, K.B., 2005. Up-regulation of acidic ribosomal phosphoprotein P0 in response to freezing or anoxia in the freeze tolerant wood frog, Rana sylvatica. Cryobiology 50, 71-82.

Yan, C., Chen, J., Li, M., Xuan, W., Su, D., You, H., Huang, Y., Chen, N., Liang, X., 2016. 
A decrease in hepatic microRNA-9 expression impairs gluconeogenesis by targeting FOXO1 in obese mice. Diabetologia 59, 1524-1532.

Ye, W., Lv, Q., Wong, C.-K.A., Hu, S., Fu, C., Hua, Z., Cai, G., Li, G., Yang, B.B., Zhang, Y., 2008. The effect of central loops in miRNA:MRE duplexes on the efficiency of miRNA-mediated gene regulation. PLoS One 3, e1719.

Yi, R., Doehle, B.P., Qin, Y., Macara, I.G., Cullen, B.R., 2005. Overexpression of Exportin 5 enhances RNA interference mediated by short hairpin RNAs and microRNAs. RNA $11,220-226$.

Zeng, Y., Sankala, H., Zhang, X., Graves, P., 2008. Phosphorylation of Argonaute 2 at serine-387 facilitates its localization to processing bodies. Biochem. J. 413, 429-436.

Zhang, B., Qiangba, Y., Shang, P., Wang, Z., Ma, J., Wang, L., Zhang, H., 2015. A comprehensive microRNA expression profile related to hypoxia adaptation in the Tibetan pig. PLoS One 10, e0143260.

Zhang, J., Storey, K.B., 2016. RBioplot: an easy-to-use R pipeline for automated statistical analysis and data visualization in molecular biology and biochemistry. PeerJ 4, e2436.

Zhang, J., Storey, K.B., 2013. Akt signaling and freezing survival in the wood frog, Rana sylvatica. Biochim. Biophys. Acta - Gen. Subj. 1830, 4828-4837.

Zhang, J., Storey, K.B., 2012. Cell cycle regulation in the freeze tolerant wood frog, Rana sylvatica. Cell Cycle 11, 1727-1742.

Zhou, B., Li, C., Qi, W., Zhang, Y., Zhang, F., Wu, J.X., Hu, Y.N., Wu, D.M., Liu, Y., Yan, T.T., Jing, Q., Liu, M.F., Zhai, Q.W., 2012. Downregulation of miR-181a upregulates sirtuin-1 (SIRT1) and improves hepatic insulin sensitivity. Diabetologia $55,2032-2043$. 


\section{CHAPTER 5}

\section{General Discussion}




\subsection{Wood frogs and freezing}

Animals living in cold environments have adapted unique strategies such as freeze tolerance for dealing with the prolonged subzero temperatures of winter. Wood frogs, and other freeze tolerant organisms, have developed specialized survival adaptations to overcome the cellular consequences of freezing. The main cellular cryoprotection strategy in place involves the rapid synthesis and distribution of small molecular weight cryoprotectants, which for wood frogs is glucose (Storey and Storey, 1985). Another hallmark freeze tolerant adaption is metabolic rate depression and reorganization. The disequilibrium between active energy requirements and the limited availability of ATP producing resources when frozen makes transitioning into a hypometabolic state a necessity that allows organisms to extend the time they can survive on internal fuel reserves (Storey and Storey, 2004).

To survive on their internal reserves, freeze tolerant animals reprioritize their metabolic functions and make a switch from aerobic ATP production to glycolytic and fermentative pathways (Storey and Storey, 2013). Despite this backdrop of metabolic depression, the wood frog is able to balance the global suppression of metabolic functions with the selective activation of genes and proteins required for freeze survival (Storey and Storey, 2017). Various molecular and biochemical mechanisms are required for the facilitation of basic survival strategies and for facilitating "pro-survival" processes. Some of these defense mechanisms that wood frogs employ include the overexpression of tissue damage repair proteins such as fibrinogen (Cai and Storey, 1997), the enhancement of antioxidant enzymes and defenses (Joanisse and Storey, 1996), and the upregulation of select stressactivated chaperone proteins (Kiss et al., 2011). 
To elicit the extensive metabolic reorganization required for freezing, the wood frog actively leverages all the transcriptional, post-transcriptional, translational, and posttranslational regulatory mechanisms (Storey and Storey, 2017). This thesis explored the role of post-transcriptional regulation that is elicited in part through the action of miRNAs, small RNAs that play a big part in gene regulation. Since their discovery in Caenorhabditis elegans 1993, microRNAs have come to the forefront as a dynamic molecular mechanism of adaptation capable of selective rapid transcriptome regulation (Lee et al., 1993). Due to the broad nature of miRNA regulation, studies have found miRNAs to be responsible for facilitating global metabolic rate depression, for selectively promoting cell survival defenses, and for maintaining cellular functions, as was posited in Chapter $\mathbf{1}$ and illustrated in Chapter 3 and 4. MicroRNAs are able to elicit this control by negatively regulating gene expression via binding to target mRNA transcripts and subsequently leading to their translational repression or degradation (Bartel, 2004). Elucidating the diverse expression patterns and biological functions under the regulatory control of miRNAs adds a new dimension to our understanding of complex gene regulatory networks and the transcriptome as a whole.

\subsection{Role of microRNAs in freeze tolerance}

MicroRNAs are a multifunctional group of regulatory molecules that can play both activating and suppressive roles in the cell (Bartel, 2004). This thesis explored whether miRNA biogenesis, expression, and function would be regulated in a tissue-specific manner to facilitate freeze survival. To explore this question, this study examined key miRNA biogenesis factors using immunoblots, performed a large-scale RT-qPCR analysis 
of miRNA expression levels, and leveraged various bioinformatics programs to characterize miRNA biological functions. While there are hundreds of conserved miRNA species that have been annotated in animals, this study was limited to a group of 113 and 109 miRNAs in wood frog brain and liver, respectively. The reason only $\sim 110$ miRNAs were examined was due to the fact that currently, the wood frog nuclear genome has not been sequenced. As such, all miRNA sequences from this study were obtained by constructing conserved miRNA primers using the $\sim 200$ miRNAs annotated in Xenopus as a scaffold for multi-sequence alignments with other related species. While genomesequencing has become more affordable and accessible- the large size of the wood frog genome, that is 2.5 times bigger than the human genome, makes sequencing very expensive $(>\$ 50,000)$ and increases the complexity of downstream assembly and annotation. Despite these massive challenges, Dr. Andor J. Kiss in Miami University is currently working to sequence the wood frog genome. His lab has recently completed the first draft assembly of the wood frog nuclear genome and he is now working on additional sequencing runs to fill in missing gaps in the genome and to set the stage for subsequent full genome de novo assembly and annotation.

Chapter 3 examined miRNA biogenesis and regulation in wood frog brain over the course of a freeze-thaw cycle. Chapter 4 was a parallel-study that explored miRNA biogenesis and regulation in wood frog liver, the metabolic and cryoprotective center, over the course of a freeze-thaw cycle. The strikingly contrasting results between these studies provides us with strong evidence that suggests the distinct tissue-specific regulation and function of miRNAs in vertebrate freeze tolerance and stress-responses. Examining the miRNA responses in these diverse tissues will help us better understand the molecular 
underpinnings that control the different transcriptional output of these tissues in response to freezing and thawing.

This study on wood frogs is the first exploration of miRNA biogenesis and regulation in the brain of a freeze-tolerant vertebrate. In frozen and thawed brains, there was a correlation between the downregulation of both the miRNAs differentially expressed during freezing and thawing and the miRNA biogenesis pathway. Immunoblotting of the key miRNA biogenesis factors found an overall decrease in protein levels potentially resulting in a reduced capacity for miRNA biogenesis and processing. The subsequent quantification of 113 miRNAs found 41 miRNAs that were differentially expressed over the course of the freeze-thaw cycle, with 39 of those miRNAs being significantly downregulated during stress. Downregulated miRNAs were found to play a role in facilitating global neuronal maintenance and aided in promoting cellular survival. The largely downregulated miRNA fingerprint observed herein suggests a miRNA-mediated cryoprotective mechanism responsible for fine tuning neuronal functions and facilitating successful whole brain freezing and thawing. These findings illustrate a novel miRNA-mediated neuroprotective mechanism that potentially allows brains to circumvent the extensive translational silencing being undertaken across the rest of the body. The pro-survival processes being "activated" by the downregulated miRNAs included; synaptic signaling, intracellular signal transduction, anoxia/ischemia protective mechanisms, and chaperone-mediated protein folding.

In contrast to the global reduction of miRNAs observed in freeze tolerant brains, the liver freeze and thaw responsive miRNA fingerprint showed an opposite pattern of enhanced miRNA biosynthesis and stress-activated upregulations. Immunoblotting demonstrated an 
increase in key miRNA biogenesis proteins which suggested an enhanced capacity for miRNA biosynthesis. This supported the predominant upregulation observed in the RTqPCR measurements of the 20 miRNAs that were differentially expressed, out of the total 109 that were evaluated. In silico downstream miRNA target prediction implicated the miRNAs in suppressing various pro-growth processes and helping to elicit a state of global metabolic rate depression. The main pathways being targeted by the upregulated miRNAs, and therefore being suppressed, included DNA replication, mRNA processing and splicing, protein synthesis and turnover, carbohydrate metabolism, etc. This newly identified enhanced capacity for miRNA biosynthesis and the miRNA mediated suppression of energy-expensive processes appears to be vital for successful hepatic freeze survival. Using miRNAs to "turn off" these energy-expensive processes helps limit the shunting of valuable and limited ATP stores from pro-growth processes that are not essential for freeze survival.

The very different miRNA responses observed in wood frog brain and liver illustrated the two main types of miRNA regulatory responses apparent in freeze tolerance; [1] reductions in miRNA levels to induce the activation of targeted pathways, and [2] increases in miRNA levels to suppress and inhibit targeted miRNA pathways (Bartel, 2004). The contrasting miRNA fingerprints measured highlight the high degree of tissue-specificity and unique metabolic needs that miRNAs are able to cater to. To asses the biological relevance of these findings and the validity of these bioinformatics predictions- downstream qPCR measurements of the miRNA targeted mRNA transcripts, as well as immunoblot measurements of the proteins they encode for should be performed. To our knowledge, this is the first report of a neuronal miRNA-mediated program for the activation and 
maintenance of living brain tissue during periods of environmental stress. The miRNAinduced neural activation and maintenance is required to ensure that after thawing, wood frogs are able to maintain/restore brain function and that they do not exhibit any neurological or behavioural deficits. This was in striking contrast to the miRNA fingerprint presented by liver that depicted a potential miRNA-mediated suppression of various energy-expensive pathways. The data for liver support metabolic rate depression as one of the main hallmarks of freeze tolerance (Storey and Storey, 2004). However, since liver is the body's metabolic center, it was interesting to find that the pattern of miRNA biogenesis and differential expression in liver illustrated a global trend of suppression. The data indicated that miRNAs functioned to selectively depress non-essential liver functions in the frozen state as well as to aid in the accumulation of cryoprotectant glucose. This hepatic miRNA-induced "off-switch" was not seen in brain. Collectively, these findings make perfect metabolic sense as they support the overall metabolic and regulatory nature of each of the brain and liver and the roles that they play during freeze/thaw.

One of the main trends observed in both tissues was that the majority of miRNAs remained unchanged over the course of the freeze-thaw cycle. This suggests that miRNA regulation is very selective and under tight regulatory control. Another parallel between the two tissues was the correlation between miRNA biogenesis and regulation. Increases in the levels of liver miRNA biogenesis proteins matched the upregulation of differentially expressed miRNAs which suggests that this response is not global but rather that the enhanced capacity for miRNA biogenesis is selectively aiding the upregulation of stressresponsive miRNAs. This newly identified "rule" was also applicable to the opposite trend observed in brain where the reduced capacity for miRNA biogenesis appeared to 
selectively reduce the abundance of stress-responsive miRNAs. While these findings appear to be rather clear-cut in Chapter 3 and 4, it should be noted that this may not always be the case, as miRNA networks are very intricate and complex. To test whether this trend is universal, miRNA biogenesis factors and miRNA expression levels should be measured in other tissues, animals, and stresses using Next-Generation Sequencing to study the global expression trend of all miRNAs.

Comparing the findings of this thesis with previous work on miRNA responses in muscles of frozen wood frogs, various parallel trends and differences can be observed. An evaluation of 53 miRNAs in cardiac and skeletal muscles over a wood frog freeze-thaw cycle found 21 cardiac miRNAs to be downregulated during thawing and 16 skeletal miRNAs to be overexpressed during freezing. Bioinformatic target prediction for heart miRNAs that decreased found them to be involved in processes such as hypertrophy, cell structure, and cardiomyopathy, all of which likely serve a cardioprotective role during freezing and thawing (Bansal et al., 2016). This response of overall miRNA downregulation during stress in hearts suggested that there would also be a miRNAmediated "activation" mechanism in place, to facilitate the quick restoration of heart function in the thawed frog. This makes metabolic sense as the resumption of heartbeat is the first observable physiological function to occur when wood frogs thaw, well before breathing and voluntary muscle movement are restored (Storey and Storey, 2017). This was similar to our observations in frozen brains in which miRNAs are likely playing an activation and maintenance role, a role that is required to cater to the tissue-specific functions of these tissues. 
The overall pattern of freeze-responsive skeletal muscle miRNAs was similar to the miRNA fingerprint seen in liver, wherein the majority of differentially expressed miRNAs were found to be upregulated (Bansal et al., 2016). Both skeletal muscle and liver freezeresponsive miRNAs mapped to cell functions that would help transition the cell into a metabolically suppressed state to conserve internal fuel reserves over the cold winter months. Taken together, we can see that the miRNA responses in wood frog cardiac and skeletal muscles, were distinctly tissue-specific and promoted activation and suppression phenotypes, respectively, as was observed here for brain and liver miRNAs. These newlyidentified freeze-responsive wood frog miRNAs join the ranks of previously categorized CryomiRs, a subset of cold-responsive miRNAs found to facilitate cold hardiness, hibernation, freeze avoidance, and freeze tolerance (Lyons et al., 2013). Indeed, studies in various natural models of environmentally-induced hypometabolism highlight the importance of miRNAs in regulating major metabolic cascades (Biggar and Storey, 2011).

\subsection{Future directions}

Although traditional dogma makes a direct link between changes in gene transcription and changes in protein production, it is now known that there are many caveats to this, such as influences of miRNA in inhibiting mRNA translation or targeting transcripts for degradation that are examined in this thesis. MicroRNA regulation during stress responses is a complex system that opens the door to various types of follow-up experiments that should be explored to better dissect the massive potential and macro effects of these miRNAs. While this study focused on the interplay between miRNAs and freezing in wood frogs, this is not the only environmental stress wood frogs are able to endure. Indeed, freeze tolerance is composed of component stresses of cellular dehydration, prolonged anoxia 
exposure, and hyperglycemia, all of which the wood frog is able to survive when frozen and when unfrozen (Storey and Storey, 2017). As such, examining the stress-specific miRNA response to each of the component stresses will allow us to determine whether there is a singular miRNA response to all stresses or whether the dynamic miRNA regulatory network changes depending on the stress with which the frogs are challenged. Wood frogs are an ideal model to answer this question as they serve as an excellent multifactorial model of environmental stress that can be used to study the separate effects of anoxia, dehydration, freezing, and hyperglycemia.

Since the brain is a complex tissue made up of multiple regions and numerous cell types, examining miRNA expression in specific brain regions using microdissection tools would be pertinent to better characterizing the observed miRNA responses. Moreover, we can further dissect this response by investigating the subcellular compartment-specific expression of miRNAs. New studies are now highlighting the functional importance of these subpopulations of organelle-specific miRNAs, and a main organelle being examined for its unique miRNA profile is the mitochondrion (Borralho et al., 2014). These 'MitomiRs' are significantly enriched in the mitochondria and have been shown to play roles in cardiovascular health and in regulating metabolic outputs (Borralho et al., 2014; Srinivasan and Das, 2015). Current work is investigating the mechanistic properties of MitomiRs; [1] how do they enter the mitochondria?, [2] does the mitochondrial genome contain uncharacterized novel mitochondrial miRNAs?, [3] how are these miRNAs processed in the mitochondria? MitomiRs can be studied using fluorescence RNA in situ hybridization (FISH) to track miRNAs in the cell and to identify their subcellular localizations. FISH will be able to reveal the miRNAs localized in organelles such as the 
mitochondria by using miRNA-specific probes consisting of complementary oligonucleotides attached to fluorescent dyes (Baker, 2012). Furthermore, one can also isolate specific organelles, extract organelle-specific RNA populations from each, and perform the necessary miRNA sample preparations and downstream qPCR measurements on the miRNAs of interest.

For a more extensive picture of the effects of miRNA regulation on the cell, one can investigate the interaction and targeting of miRNAs and their effects on targeted transcripts. While the present large-scale studies in this thesis were able to quantitate the relative expression levels of $\sim 110$ miRNAs, more advanced next generation sequencing would allow us to analyze the full miRNA fingerprint of an organ, a fingerprint that consists of several hundred conserved miRNAs and novel miRNAs. This can be challenging as the wood frog is not genome-sequenced, making annotation and analysis very difficult since numerous ingenious steps would be required to overcome this problem. In an effort to identify and characterize novel species-specific miRNAs in wood frogs, I used previously sequenced wood frog small RNA transcriptomes and various bioinformatics tools, including the machine-learning prediction tool MIRDEEP2, to evaluate all RNA sequences for the suite of characteristics that define miRNA (Friedländer et al., 2008). The analysis of three wood frog small RNA-Sequencing transcriptomes lead to the discovery of 32 novel wood frog specific miRNAs. A detailed breakdown of how this analysis was performed can be found in Appendix G. Precursor and mature sequences of the 32 novel miRNAs are annotated in Appendix $\boldsymbol{H}$.

To validate that these novel miRNAs are indeed "real" I designed miRNA specific primers for them and examined their expression levels in pooled samples of control, $24 \mathrm{~h}$ frozen, 
and $8 \mathrm{~h}$ thawed wood frog brain and liver. Novel wood frog miRNA primers are listed in Appendix E. Future work will probe much deeper into examining tissue and stress-specific responses, characterizing these novel miRNAs, and identifying their biological functions using in silico bioinformatics prediction tools. MicroRNA target predictions can be experimentally validated using luciferase promoter assays. Briefly, using a vector with a luciferase reporter constructed to be under the control of a weak promoter, subtle changes in gene expression can be observed when a miRNA of interest is added and binds to the predicted mRNA 3'UTR of interest (Jin et al., 2013). Furthermore, to examine the effects of these miRNAs in the cell, one can use qPCR to measure the levels of the predicted mRNA targets and this can be coupled with immunoblot measurements of the downstream proteins that the targeted mRNA targets encode.

Another factor to be considered in future studies is the protection that is offered to normally labile mRNA molecules by stabilizing and storing transcripts in subcellular granules. In the case of freezing, this could allow mRNAs that are not needed in the hypometabolic state to be preserved until their translation is reinitiated when the animal thaws. There is substantial evidence that this occurs during hypometabolism. Studies on hibernating squirrels investigated the distribution of individual mRNA transcript types between polysome and monosome fractions of tissues in aroused vs torpid squirrels (Hittel and Storey, 2002) and another imaging study showed the intracellular formation of large numbers of stress granules and/or P-bodies containing translationally-stalled mRNA transcripts (Tessier et al., 2014). It would be interesting to explore what happens when freezing is used to conserve energy. Are most mRNA transcripts temporarily silenced or are they targeted for degradation? This question could be answered by conducting an in- 
depth study of the abundance, localization, and identity of stress granules and P-bodies, two types of non-membrane-bound ribonucleoprotein (RNP) granules that can accumulate and store mRNAs (Kedersha et al., 2005).

Stress granules are cytoplasmic messenger RNP granules composed of mRNAs stalled at translation initiation, numerous translation initiation factors, and RNA-binding proteins (RBPs) (Jain et al., 2016). P-bodies are comprised of mRNAs associated with translational repressors and mRNA decay machinery (Aizer et al., 2014). Cytoplasmic RBPs like TIA1/R and PABP-1 are localized to stress granules under cell stress conditions where they promote translational silencing (Decker and Parker, 2012). Indeed, work in hibernating ground squirrel liver revealed that levels of the stress granule-specific TIA- $1 \mathrm{a}$ and TIA- $1 \mathrm{~b}$ proteins in the nucleus rose by 7- and 4-fold, respectively, during prolonged hibernation (Tessier et al., 2014). In addition, the percentage of cells that showed subnuclear foci that immunostained positively for TIA-1 or PABP-1 rose from $1-2 \%$ in euthermic squirrels to $>95 \%$ in hibernating animals indicating extensive storage of mRNA transcripts during torpor. These explorative studies in hibernating mammals suggest that similar mechanisms might be leveraged in freeze tolerant vertebrates. Indeed, similar immunohistochemistry and/or FISH can be used to elucidate the RNA landscape in frozen frogs. Future studies can analyze the fate of mRNA transcripts in frozen wood frog brain and liver through the use of RNA immunoprecipitation (RIP) of stress granule specific (TIA-1) and P-body specific (Pat1/PatL1) proteins with co-isolation of the RNA species associated with these proteins. This can then be combined with total RNA sequencing (RIP-Seq) to characterize the identities of specific RNA molecules that are associated with these two RNA-binding 
proteins in control vs frozen vs thawed states. Alternatively, a more focused approach can be applied using RIP-qPCR to amplify specific mRNA and miRNA transcripts.

\subsection{Significance and applications}

Unravelling the mechanisms by which animals are able to survive prolonged periods of stress can potentially lead to the identification of novel therapeutic and cryoprotective targets. By identifying the miRNAs critical for freeze tolerance and other extreme hypometabolic states, we can determine which miRNAs are necessary for basic cellular survival and which miRNAs instigate cellular protective programs. Current work is examining the clinical and therapeutic role of microRNAs in infectious diseases, cancer, neurodegenerative disorders, and a myriad of other conditions (Broderick and Zamore, 2011; Hayes et al., 2014; Wang et al., 2016). The freeze-responsive brain miRNAs identified herein can also serve as targets for the neuronal adaptations and maintenance of brain functions. To date, neuropathological studies have shown that miRNAs can serve as promising candidates for various neurodegenerative diseases (Kuss and Chen, 2008). For example, miR-34, a miRNA that has been previously implicated in several physiological processes, appears to be overexpressed in the brains of Alzheimer's patients where it acts to regulate p53 expression and the subsequent phosphorylation of tau tangles (Hooper et al., 2007). In transgenic mouse models of this condition, the downregulation of miR-34 was able to rescue some cognitive abilities (Zovoilis et al., 2011).

MicroRNAs are also being identified as biomarkers for various diseases and conditions including nervous system disorders, cancer, diabetes, viral infections, etc. (Hayes et al., 2014; Wang et al., 2016). This has been made possible by both NGS sequencing and by 
the development of new bioinformatics tools such as the newly-developed program I coauthored, RBioFS. This program leverages machine-learning technology to accurately identify potential miRNA biomarkers from various medical and comparative datasets (Zhang et al., 2016) (http://kenstoreylab.com/?page_id=2542).

The miRNAs characterized in this thesis, particularly in the liver, could be further investigated as potential miRNA candidates for inducing metabolic rate depression. This idea is of great interest to various medical fields, the main one being organ preservation and storage. If we can use miRNAs to help induce a global state of dormancy and hypometabolism then we might be able to preserve organs for longer periods of time. This will extend the window of time available for organ transfer from donor to recipient and thereby greatly increase the number of donor organs that can be successfully transplanted, with the potential to save millions of lives. Moreover, miRNAs are also being investigated for their tissue regenerative properties and our findings in wood frog brains highlight potential miRNA candidates that could possibly help maintain and enhance overall cellular and neuronal functions. A study on a rat model of skeletal muscle injury found that the local injection of muscle-specific miRNAs promoted an acceleration of muscle regeneration and healing (Nakasa et al., 2010). Interestingly, the miRNAs used in this study included miR-1 that was found to be significantly upregulated in the muscles of hypometabolic hibernating marsupials and bats, where they were suggested to aid muscle remodelling and prevention of muscle disuse atrophy during torpor (Hadj-Moussa et al., 2016; Kornfeld et al., 2012). This observation emphasizes the importance of applying our findings from animals that can push the elastic-limits of survival into new and innovative therapeutics. 
Such medical studies could investigate the use of exogenous miRNAs or anti-miRs to simulate the putatively protective metabolic responses observed in frozen wood frog brain and liver. Generally, miRNA upregulation is achieved through the administration of synthetic exogenous miRNAs or though the administration of miRNA expressing vectors whereas miRNA downregulation is mimicked through the addition of anti-sense nucleotides, often chemically modified to ensure stability and specificity (Ishida and Selaru, 2013). Another approach to inhibit specific miRNAs is through the use of antimiRs. Anti-miRs are miRNA-sponges that seek to occupy mature miRNA using DNA sequences, typically plasmids that express target mRNA binding sites in high copy numbers (Zhang et al., 2013). These miRNA-based interventions and therapeutics can be administered via local microinjections, microbubbles, nanoparticles, lipid-vesicles, etc. (Zhang et al., 2013). Taken together, the identification of miRNAs and pathway targets in this study sets the stage for numerous innovative future medical applications that just maybe might work!

\subsection{Summary}

To survive in a frozen state of suspended animation, wood frogs undergo extensive wholebody reorganization of the cellular, biochemical, and molecular landscapes through the coordination of all regulatory levels. This thesis demonstrated that the post-transcriptional regulation orchestrated by miRNAs appears to be playing an important and dynamic role in facilitating freeze tolerance. MicroRNA expression was found to be highly tissuespecific and the patterns illustrated in brain and liver were contrasting, with each of the chapters highlighting one of the two main regulatory outputs of miRNA. Brain freeze-thaw 
specific miRNAs were found to play an "activating" role where they are suggested to serve a neuroprotective role by maintaining neuronal tissues, preserving synaptic functions, and circumventing translational repression in the frozen state. The global downregulation of differentially expressed brain miRNAs was coupled with an overall decreased capacity for miRNA biogenesis. In contrast, differentially expressed liver freeze-thaw specific miRNAs were upregulated, which was supported by the observed overall increase of miRNA biogenesis capacity. Bioinformatics target enrichment lead to the identification of a novel miRNA-mediated mechanism for facilitating global hepatic metabolic rate depression and energy conservation strategies. This thesis also demonstrated that the tight regulation of miRNA biogenesis in both tissues was reflected in the miRNA fingerprint of each tissue, where increased miRNA biogenesis capacity translated to an overall upregulation of select miRNA species, and vice versa. These findings highlight the critical role of miRNAs in regulating brain and liver metabolic reorganization and survival over the course of the freeze-thaw cycle, emphasize the complex interconnectivity of the various regulatory layers, and set the stage for future innovative miRNA-based therapeutics. 


\section{REFERENCES [CHAPTER 5]}

Aizer, A., Kalo, A., Kafri, P., Shraga, A., Ben-Yishay, R., Jacob, A., Kinor, N., Shav-Tal, Y., 2014. Quantifying mRNA targeting to P-bodies in living human cells reveals their dual role in mRNA decay and storage. J. Cell Sci. 127, 4443-4456.

Baker, M., 2012. RNA imaging in situ. Nat. Methods 9, 787-790.

Bansal, S., Luu, B.E., Storey, K.B., 2016. MicroRNA regulation in heart and skeletal muscle over the freeze-thaw cycle in the freeze tolerant wood frog. J. Comp. Physiol. B 186, 229-241.

Bartel, D.P., 2004. MicroRNAs genomics, biogenesis, mechanism, and function. Cell 116, 281-297.

Biggar, K.K., Storey, K.B., 2011. The emerging roles of microRNAs in the molecular responses of metabolic rate depression. J. Mol. Cell Biol. 3, 167-175.

Borralho, P.M., Rodrigues, C.M.P., Steer, C.J., 2014. Mitochondrial MicroRNAs and Their Potential Role in Cell Function. Curr. Pathobiol. Rep. 2, 123-132.

Broderick, J.A., Zamore, P.D., 2011. MicroRNA therapeutics. Gene Ther. 18, 1104-1110.

Cai, Q., Storey, K.B., 1997. Upregulation of a novel gene by freezing exposure in the freeze-tolerant wood frog (Rana sylvatica). Gene 198, 305-12.

Decker, C.J., Parker, R., 2012. P-Bodies and Stress Granules: Possible Roles in the Control of Translation and mRNA Degradation. Cold Spring Harb. Perspect. Biol. 4, a012286-a012286.

Friedländer, M.R., Chen, W., Adamidi, C., Maaskola, J., Einspanier, R., Knespel, S., Rajewsky, N., 2008. Discovering microRNAs from deep sequencing data using miRDeep. Nat. Biotechnol. 26, 407-415.

Hadj-Moussa, H., Moggridge, J.A., Luu, B.E., Quintero-Galvis, J.F., Gaitán-Espitia, J.D., Nespolo, R.F., Storey, K.B., 2016. The hibernating South American marsupial, Dromiciops gliroides, displays torpor-sensitive microRNA expression patterns. Sci. Rep. 6, 24627.

Hayes, J., Peruzzi, P.P., Lawler, S., 2014. MicroRNAs in cancer: biomarkers, functions and therapy. Trends Mol. Med. 20, 460-469.

Hittel, D., Storey, K.B., 2002. The translation state of differentially expressed mRNAs in the hibernating 13-lined ground squirrel (Spermophilus tridecemlineatus). Arch. Biochem. Biophys. 401, 244-254.

Hooper, C., Meimaridou, E., Tavassoli, M., Melino, G., Lovestone, S., Killick, R., 2007. p53 is upregulated in Alzheimer's disease and induces tau phosphorylation in HEK293a cells. Neurosci. Lett. 418, 34-37.

Ishida, M., Selaru, F.M., 2013. miRNA-Based Therapeutic Strategies. Curr. Anesthesiol. Rep. 1, 63-70. 
Jain, S., Wheeler, J., Walters, R., Agrawal, A., Barsic, A., Parker, R., 2016. ATPaseModulated Stress Granules Contain a Diverse Proteome and Substructure. Cell 164, 487-498.

Jin, Y., Chen, Z., Liu, X., Zhou, X., 2013. Evaluating the microRNA targeting sites by luciferase reporter gene assay. Methods Mol. Biol. 936, 117-27.

Joanisse, D.R., Storey, K.B., 1996. Oxidative damage and antioxidants in Rana sylvatica, the freeze-tolerant wood frog. Am. J. Physiol. 271, R545-53.

Kedersha, N., Stoecklin, G., Ayodele, M., Yacono, P., Lykke-Andersen, J., Fritzler, M.J., Scheuner, D., Kaufman, R.J., Golan, D.E., Anderson, P., 2005. Stress granules and processing bodies are dynamically linked sites of mRNP remodeling. J. Cell Biol. $169,871-884$.

Kiss, A.J., Muir, T.J., Lee, Jr, R.E., Costanzo, J.P., 2011. Seasonal Variation in the Hepatoproteome of the Dehydration- and Freeze-Tolerant Wood Frog, Rana sylvatica. Int. J. Mol. Sci. 12, 8406-8414.

Kornfeld, S.F., Biggar, K.K., Storey, K.B., 2012. Differential expression of mature microRNAs involved in muscle maintenance of hibernating little brown bats, Myotis lucifugus: a model of muscle atrophy resistance. Genomics. Proteomics Bioinformatics 10, 295-301.

Kuss, A.W., Chen, W., 2008. MicroRNAs in brain function and disease. Curr. Neurol. Neurosci. Rep. 8, 190-7.

Lee, R.C., Feinbaum, R.L., Ambros, V., 1993. The C. elegans heterochronic gene lin-4 encodes small RNAs with antisense complementarity to lin-14. Cell 75, 843-54.

Lyons, P.J., Lang-Ouellette, D., Morin, P.J., 2013. CryomiRs: Towards the identification of a cold-associated family of microRNAs. Comp. Biochem. Physiol. Part D Genomics Proteomics 8, 358-364.

Nakasa, T., Ishikawa, M., Shi, M., Shibuya, H., Adachi, N., Ochi, M., 2010. Acceleration of muscle regeneration by local injection of muscle-specific microRNAs in rat skeletal muscle injury model. J. Cell. Mol. Med. 14, 2495-2505.

Srinivasan, H., Das, S., 2015. Mitochondrial miRNA (MitomiR): a new player in cardiovascular health 1. Can. J. Physiol. Pharmacol. 93, 855-861.

Storey, J.M., Storey, K.B., 1985. Triggering of cryoprotectant synthesis by the initiation of ice nucleation in the freeze tolerant frog, Rana sylvatica. J. Comp. Physiol. B 156, 191-195.

Storey, K.B., Storey, J.M., 2017. Molecular physiology of freeze tolerance in vertebrates. Physiol. Rev. 97, 623-665.

Storey, K.B., Storey, J.M., 2013. Molecular biology of freezing tolerance. Compr. Physiol. 3, 1283-308.

Storey, K.B., Storey, J.M., 2004. Metabolic rate depression in animals: transcriptional and 
translational controls. Biol. Rev. Camb. Philos. Soc. 79, 207-33.

Tessier, S.N., Audas, T.E., Wu, C.-W., Lee, S., Storey, K.B., 2014. The involvement of mRNA processing factors TIA-1, TIAR, and PABP-1 during mammalian hibernation. Cell Stress Chaperones 19, 813-825.

Wang, J., Chen, J., Sen, S., 2016. MicroRNA as Biomarkers and Diagnostics. J. Cell. Physiol. 231, 25-30.

Zhang, J., Hadj-Moussa, H., Storey, K.B., 2016. Current progress of high-throughput microRNA differential expression analysis and random forest gene selection for model and non-model systems: an R implementation. J. Integr. Bioinform. 13, 306.

Zhang, Y., Wang, Z., Gemeinhart, R.A., 2013. Progress in microRNA delivery. J. Control. Release 172, 962-74.

Zovoilis, A., Agbemenyah, H.Y., Agis-Balboa, R.C., Stilling, R.M., Edbauer, D., Rao, P., Farinelli, L., Delalle, I., Schmitt, A., Falkai, P., Bahari-Javan, S., Burkhardt, S., Sananbenesi, F., Fischer, A., 2011. microRNA-34c is a novel target to treat dementias. EMBO J. 30, 4299-4308. 
APPENDICES 


\section{APPENDIX A}

Publications, Conferences, and Grants 


\section{PUBLICATIONS [9]}

$\underline{\text { Articles published in peer-reviewed journals }}$

Review Articles [1]

Zhang, J., Hadj-Moussa, H., and Storey, K.B. (2016). Current progress of high-throughput microRNA differential expression analysis and random forest gene selection for model and non-model systems: an $\mathrm{R}$ implementation. Journal of Integrative Bioinformatics, 13(5), 306.

Journal Articles [5]

Aguilar, Oscar A., Hanane Hadj-Moussa, and Kenneth B. Storey. (2017). Freezeresponsive regulation of MEF2 proteins and downstream gene networks in muscles of the wood frog, Rana sylvatica. Journal of Thermal Biology. 67: 1-8.

Aguilar, Oscar A., Hanane Hadj-Moussa, and Kenneth B. Storey. (2016). Regulation of SMAD transcription factors during freezing in the freeze tolerant wood frog, Rana sylvatica. Comparative Biochemistry and Physiology Part B: Biochemistry and Molecular Biology. 201: 64-71.

Hadj-Moussa, H., Moggridge, J.A., Luu, B.E., Quintero-Galvis, J.F., Gaitán-Espitia, J.D., Nespolo, R.F., and Storey, K.B. (2016). The hibernating South American marsupial, Dromiciops gliroides, displays torpor-sensitive microRNA expression patterns. Scientific Reports. 6: 24627.

Liu, J., Hagberg, I., Novitsky, L., Hadj-Moussa, H., and Avis, T.J.. (2014). Interaction of antimicrobial cyclic lipopeptides from Bacillus subtilis influences their effect on spore germination and membrane permeability in fungal plant pathogens. Fungal Biology. 118: 855-861.

Katzenback, B.A., Holden, H.A., Falardeau, J., Childers, C.L., Hadj-Moussa, H., Avis, T.J., and Storey, K.B. (2014). Regulation of the Rana sylvatica brevinin-1SY antimicrobial peptide during development and in dorsal and ventral skin in response to freezing, anoxia, and dehydration. Journal of Experimental Biology. 217(8): $1392-1401$.

Articles submitted to peer-reviewed journals [1]

Hoyeck, M.P., Hadj-Moussa, H., and Storey, K.B. (2017). The role of MEF2 transcription factors in dehydration and anoxia survival in Rana sylvatica skeletal muscle, Rana sylvatica. PeerJ. 
Articles awaiting submission to peer-reviewed journals [2]

Hadj-Moussa, H. and Storey, K.B. (2017). Micromanaging freeze tolerance: The biogenesis and regulation of neuroprotective microRNAs in frozen brains. $B B A$ Gene Regulatory Networks.

Hadj-Moussa, H. and Storey, K.B. (2017). Biogenesis and regulation of the freeze-thaw responsive microRNA fingerprint in wood frog liver. Journal of Molecular and Cell Biology.

\section{OTHER PEER-REVIEWED CONTRIBUTIONS [2]}

Hadj-Moussa, H., \& Storey, K.B. (2016) Micromanaging freeze tolerance: The role of microRNAs in regulating brain cryoprotection in freeze tolerant wood frogs. Cryobiology. 3(73), 427. (Abstract)

Katzenback, B.A., Holden, H.A., Falardeau, J., Childers, C.L., Hadj-Moussa, H., Avis, T.J., \& Storey, K.B. (2013) Regulation of the antimicrobial peptide brevinin-1SY in the skin of Rana sylvatica in response to environmental stress. Cryobiology. 67(3), 441 (Abstract).

\section{SCIENTIFIC MEETING COMMUNICATIONS [12]}

(* - indicates presenting author)

14. Hadj-Moussa, H., Moggridge, J.A., Luu, B.E., Quintero-Galvis, J.F., Gaitán-Espitia, J., Nespolo, R.F., and Storey, K.B. (2015). The hibernating South American marsupial, Dromiciops gliroides, displays torpor-sensitive microRNA expression patterns. Lung health and disease across age, environment and species, Gordon Research Conference, Colby-Sawyer College, New London, NH, Aug. 20-24, 2017. International conference.

13. Hadj-Moussa, H., Moggridge, J.A., Luu, B.E., Quintero-Galvis, J.F., Gaitán-Espitia, J., Nespolo, R.F., and Storey, K.B. (2015). The hibernating South American marsupial, Dromiciops gliroides, displays torpor-sensitive microRNA expression patterns. Organ Banking Summit, Harvard University, Boston, Aug. 3-6, 2017. International conference.

12. Hadj-Moussa, H., and Storey K.B. The biogenesis and regulation of microRNAs in a natural model of vertebrate freeze tolerance. Toronto RNA enthusiasts Day (TReND). August 2, 2017. Provincial conference. 
11. Hadj-Moussa, H., and Storey, K.B. The biogenesis and regulation of microRNAs in a natural model of vertebrate freeze tolerance. Canadian Society of Molecular Biosciences. Ottawa, ON, May 16-20, 2017. National conference.

10. Hadj-Moussa, H., Moggridge, J.A., Luu, B.E., Quintero-Galvis, J.F., Gaitán-Espitia, J.D., Nespolo, R.F., Storey, K.B. The hibernating South American marsupial, Dromiciops gliroides, displays torpor-sensitive microRNA expression patterns. Canadian Society of Zoologists, Satellite Symposium: 50 years of comparative biochemistry - the legacy of Peter Hochachka. University of Manitoba, Winnipeg, MB, May 13-14, 2017. National conference.

9. Hadj-Moussa, H.* and Storey, K.B. (2016). Micromanaging Freeze Tolerance: The Role of MicroRNAs in Regulating Brain Cryoprotection. $53^{\text {rd }}$ Annual Meeting of the Society for Cryobiology (CRYO2016), Ottawa, Canada. Invited speaker. International conference.

8. Hadj-Moussa, H.*, Moggridge, J.A., Luu, B.E., Quintero-Galvis, J.F., Gaitán-Espitia, J.D., Nespolo, R.F., Storey, K.B. The hibernating South American marsupial, Dromiciops gliroides, displays torpor-sensitive microRNA expression patterns. Ottawa-Carleton Institute for Biology Symposium. May 5, 2016. Poster. Local conference.

7. Hadj-Moussa, H.*, Moggridge, J.A., Luu, B.E., Quintero-Galvis, J.F., Gaitán-Espitia, J., Nespolo, R.F., and Storey, K.B. (2015). The hibernating South American marsupial, Dromiciops gliroides, displays torpor-sensitive microRNA expression patterns. $18^{\text {th }}$ Annual Chemistry \& Biochemistry Graduate Research Conference, Concordia Univ., Nov. 20, 2015. Poster. Provincial conference.

6. Hadj-Moussa, H.* and Storey, K.B. (2015). Regulation of the central metabolic switch, PGC-1a, and interacting transcription factors in frozen and anoxic Rana sylvatica. $28^{\text {th }}$ Annual Ontario Biology Day Conference. Poster. Winner of the Cellular and Molecular Biology Poster Prize. Provincial conference.

5. Hadj-Moussa, H.* and Storey, K.B. (2015). Regulation of the central metabolic switch, PGC-1a, and interacting transcription factors in frozen and anoxic Rana sylvatica. Ottawa-Carleton Institute for Biology Symposium. Poster. Local conference.

4. Hadj-Moussa, H.* and Storey, K.B. (2015). Regulation of the central metabolic switch, PGC-1a, and interacting transcription factors in frozen and anoxic Rana sylvatica. Carleton University Undergraduate Research Day. Poster.

3. Katzenback, B.A.*, Holden, H.A., Falardeau, J., Childers, C.L., Hadj-Moussa, H., Avis, T.J., and Storey, K.B. (2014). Rana sylvatica brevinin-1SY: regulation of an 
antimicrobial peptide in response to environmental stress. Montreal, Quebec. Canadian Society of Zoologists (CSZ14), 2014-05-25. Poster. National conference.

2. Katzenback, B.A., Holden, H.A., Falardeau, J., Childers, C.L., Hadj-Moussa, H., Avis, T.J., and Storey, K.B.* (2013). Regulation of the antimicrobial peptide brevinin1SY in the skin of Rana sylvatica in response to environmental stress. Cryobiology: International Journal of Low Temperature Biology and Medicine. Society for Cryobiology's 50th Annual Meeting (CRYO2013), 2013-07-28. Poster. International conference.

1. Katzenback, B.A., Holden, H.A., Falardeau, J., Childers, C.L., Hadj-Moussa, H., Avis, T.J.*, and Storey, K.B. (2013). Regulation of the antimicrobial peptide brevinin$1 \mathrm{SY}$ in the skin of Rana sylvatica in response to environmental stress. Canadian Society of Microbiologists 63rd Annual Conference. 63rd Annual Conference of the Canadian Society of Microbiologists (CSM), 2013-06-17 (84). Poster. National conference.

\section{GRANTS [1]}

Experiment - Crowd sourcing mini-grant for hypoxic Red Devil squid miRNA studies

Competed and won in the 'Animal Superpower Grant Challenge' hosted by Experiment.com crowd-funding science platform. Was awarded a mini-grant titled "The Red Devil squid: Oxygen deprived, cold, and under pressure" to study the microRNA regulation of hypoxic deep-sea squid. (www.experiment.com/toughsquid) 


\section{APPENDIX B}

Antibody Supplier Information 
Table B1. Antibody information and suppliers.

\begin{tabular}{|c|c|c|c|}
\hline Antibody & Company & Catalogue number & Reactivity \\
\hline DROSHA & NeoBiolab & A8336 & Anti-Rabbit \\
\hline DGCR8 & GeneTex & GTX130061 & Anti-Rabbit \\
\hline XPO5 & GeneTex & GTX130727 & Anti-Rabbit \\
\hline $\mathbf{R A N}$ & GeneTex & GTX114139 & Anti-Rabbit \\
\hline DICER & SantaCruz & SC-30226 & Anti-Rabbit \\
\hline TRBP & GeneTex & GTX48546 & Anti-Rabbit \\
\hline PACT & GeneTex & GTX114215 & Anti-Rabbit \\
\hline AGO1 & GeneTex & GTX47799 & Anti-Rabbit \\
\hline AGO2 & ECM BioSciences & AP5281 & Anti-Rabbit \\
\hline p-AGO2 Tyr393 & ECM BioSciences & AP5311 & Anti-Rabbit \\
\hline p-AGO2 ${ }^{\text {Ser387 }}$ & ECM BioSciences & AP5291 & Anti-Rabbit \\
\hline
\end{tabular}




\section{APPENDIX C}

\section{Verifying Antibody Specificity for Immunoblotting}


To ensure that the antibodies used cross-reacted with the protein of interest at the appropriate molecular weight, the following steps were taken:

1. When companies supplied their antibody epitope sequence, this was aligned with various species (human, mouse, chicken, Xenopus) using CLUSTAL OMEGA to determine if the epitope region is conserved which would increase the likelihood that the protein is also conserved in the wood frog. Antibodies that recognized highly conserved epitopes were used.

2. When possible, polyclonal antibodies that were tested on numerous species were used as to increase the chance of cross-reactivity in the wood frog.

3. Immunoblot membranes were incubated with milk to minimize non-specific background binding, prior to primary antibody probing

4. All antibodies were tested with a mammalian positive control sample (thirteenlined ground squirrel), to verify that the antibodies cross-react at the expected molecular weight. 
Figure C1. Representative protein multi-sequence alignment of antibody epitope.

The multi-sequence protein alignments of DICER below depicts antibody epitope mapping between amino acids 1698-1729 (highlighted in yellow) near the C-terminus of DICER human dicer. The alignment was generated using CLUSTAL OMEGA (1.2.4).

The alignment was generated using sequenced from Xenopus tropicalis, Xenopus laevis, human, and mouse.

\begin{tabular}{|c|c|c|}
\hline X.tropicalis & nypfknkayllqafthasyhyntitdcyqrleflgdaildylitkhlyedprqhspgvlt & 1704 \\
\hline X.laevis & nypfknkayllqafthasyhyntitdcyqrleflgdaildylitkhlyedprqhspgvlt & 1702 \\
\hline Human & nyrfknkayllqafthasyhyntitdcyqrleflgdaildylitkhlyedprqhspgvlt & 1733 \\
\hline Mouse & 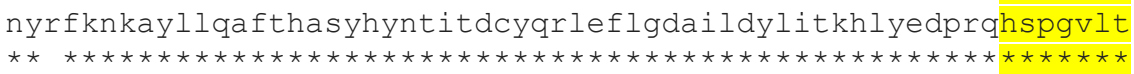 & 1717 \\
\hline x.tropicalis & dlrsalvnntifaslavkydyhkyfkaispelfhviddfvqfqleknemqgmdselrrse & 4 \\
\hline X.laevis & dlrsalvnntifaslavkydyhkyfkaispelfhviddfvqfqleknemqgmdselrrse & 52 \\
\hline Human & dlrsalvnntifaslavkydyhkyfkavspel fhviddfvqfqleknemggmdselrrse & 3 \\
\hline Mouse & 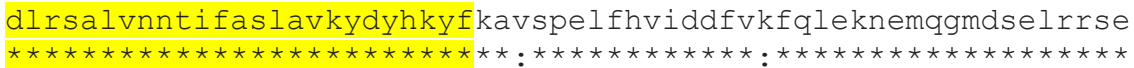 & \\
\hline
\end{tabular}


Figure C2. Representative whole image of ECL-detected membrane using control, $24 \mathrm{hr}$ frozen, and $8 \mathrm{hr}$ thawed wood frog liver samples probed with anti-DGCR8 antibody.

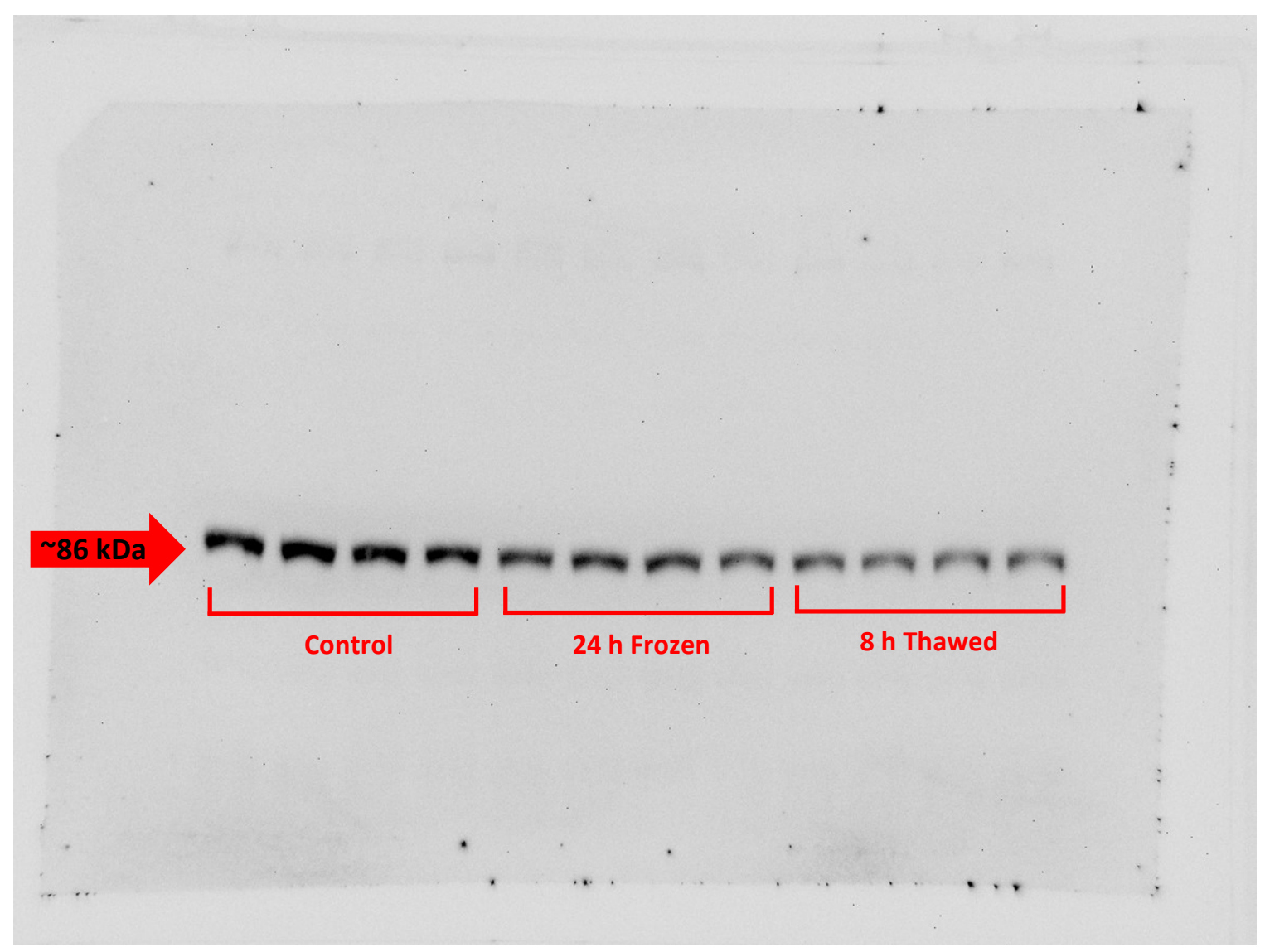




\section{APPENDIX D}

\section{Optimized Immunoblotting Conditions}


Table D1. Optimized immunoblotting conditions used in this thesis.

\begin{tabular}{|c|c|c|c|c|}
\hline Antibody & Tissue & Protein $(\mu g)$ & Gel (\%) & Secondary Antibody (1:8000) \\
\hline \multirow[t]{2}{*}{ DROSHA } & Brain & 25 & 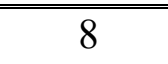 & Goat Anti-Rabbit \\
\hline & Liver & 25 & 8 & Goat Anti-Rabbit \\
\hline \multirow[t]{2}{*}{ DGCR8 } & Brain & 25 & 8 & Goat Anti-Rabbit \\
\hline & Liver & 25 & 8 & Goat Anti-Rabbit \\
\hline \multirow[t]{2}{*}{ EXPORTIN-5 } & Brain & 25 & 8 & Goat Anti-Rabbit \\
\hline & Liver & 25 & 8 & Goat Anti-Rabbit \\
\hline \multirow[t]{2}{*}{$\mathbf{R A N}$} & Brain & 25 & 15 & Goat Anti-Rabbit \\
\hline & Liver & 25 & 15 & Goat Anti-Rabbit \\
\hline \multirow[t]{2}{*}{ DICER } & Brain & 40 & 6 & Goat Anti-Rabbit \\
\hline & Liver & 40 & 6 & Goat Anti-Rabbit \\
\hline \multirow[t]{2}{*}{ TRBP } & Brain & 25 & 12 & Goat Anti-Rabbit \\
\hline & Liver & 25 & 12 & Goat Anti-Rabbit \\
\hline \multirow[t]{2}{*}{ PACT } & Brain & 25 & 12 & Goat Anti-Rabbit \\
\hline & Liver & 25 & 12 & Goat Anti-Rabbit \\
\hline \multirow[t]{2}{*}{ AGO1 } & Brain & 25 & 8 & Goat Anti-Rabbit \\
\hline & Liver & 25 & 8 & Goat Anti-Rabbit \\
\hline \multirow[t]{2}{*}{ AGO2 } & Brain & 25 & 8 & Goat Anti-Rabbit \\
\hline & Liver & 25 & 8 & Goat Anti-Rabbit \\
\hline \multirow[t]{2}{*}{ p-AGO2 Tyr393 } & Brain & 25 & 8 & Goat Anti-Rabbit \\
\hline & Liver & 25 & 8 & Goat Anti-Rabbit \\
\hline p-AGO2 ${ }^{\text {Ser387 }}$ & Liver & 25 & 8 & Goat Anti-Rabbit \\
\hline
\end{tabular}


APPENDIX E

Primer Sequences 
Table E1. Primers used for analysis of conserved miRNA expression in the brain and liver of $R$. sylvatica, including miRNA-specific forward primers, universal reverse primer, and the stem-loop adapter for reverse transcription.

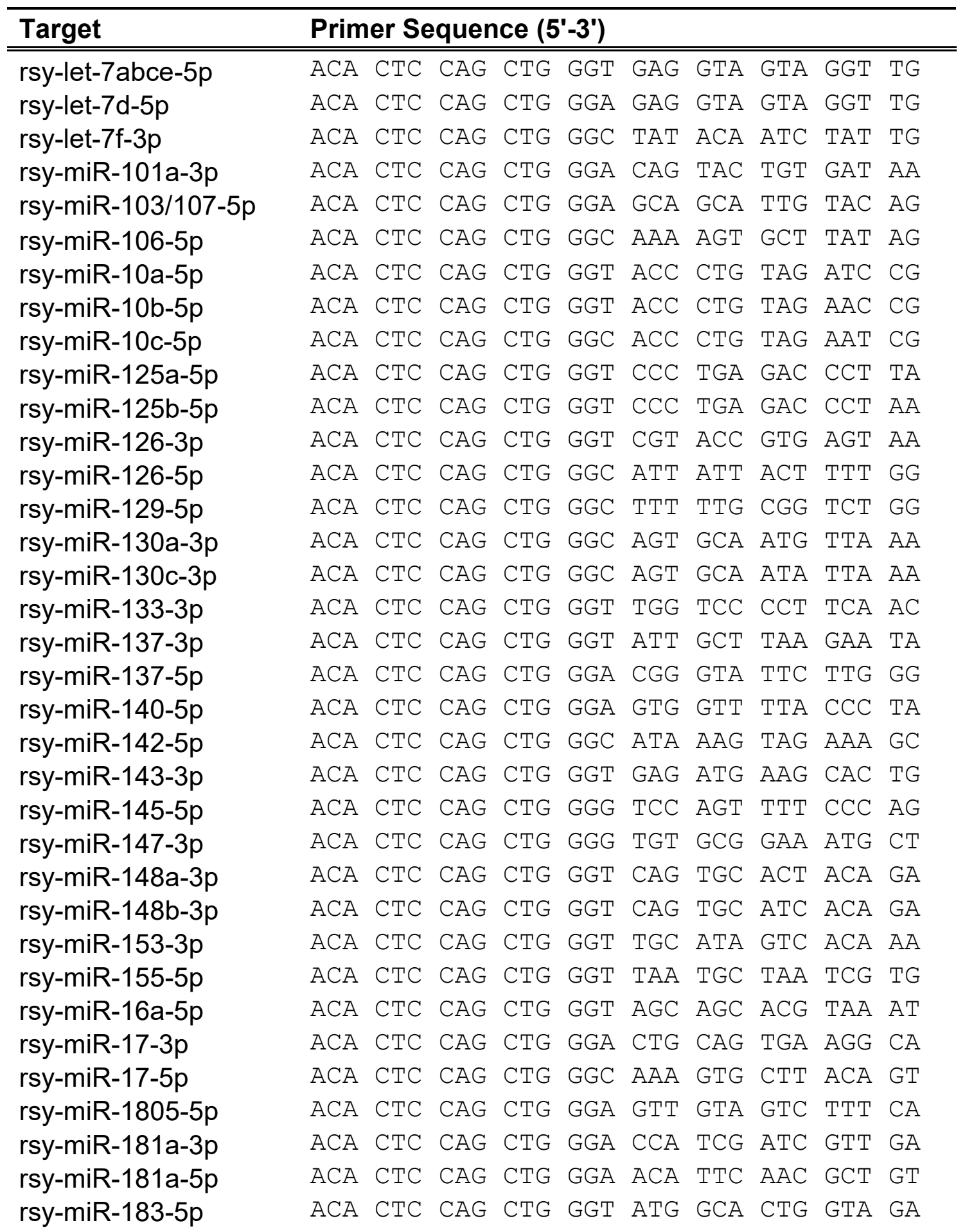


rsy-miR-184-3p

rsy-miR-18-5p

rsy-miR-187-3p

rsy-miR-191-5p

rsy-miR-192-5p

rsy-miR-193-3p

rsy-miR-194-5p

rsy-miR-196b-5p

rsy-miR-199a-5p

rsy-miR-199b-5p

rsy-miR-19a-3p

rsy-miR-19b-3p

rsy-miR-1a-3p

rsy-miR-200a-3p

rsy-miR-200b-3p

rsy-miR-202-3p

rsy-miR-203-3p

rsy-miR-204-5p

rsy-miR-205-5p

rsy-miR-206-3p

rsy-miR-208-3p

rsy-miR-20a-5p

rsy-miR-20b-5p

rsy-miR-210-3p

rsy-miR-212-3p

rsy-miR-214-3p

rsy-miR-21-5p

rsy-miR-216-5p

rsy-miR-2184-5p

rsy-miR-2188-5p

rsy-miR-221-3p

rsy-miR-222-3p

rsy-miR-223-3p

rsy-miR-22-3p

rsy-miR-22-5p

rsy-miR-23-3p

rsy-miR-24a-5p

rsy-miR-24b-3p

rsy-miR-25-3p

rsy-miR-26-3p

rsy-miR-26-5p

rsy-miR-27-3p

rsy-miR-2970-5p
ACA CTC CAG CTG GGT GGA CGG AGA ACT GA ACA CTC CAG CTG GGT AAG GTG CAT CTA GT ACA CTC CAG CTG GGT CGT GTC TTG TGT TG ACA CTC CAG CTG GGC AAC GGA ATC CCA AA ACA CTC CAG CTG GGA TGA CCT ATG AAT TG ACA CTC CAG CTG GGA ACT GGC CCG CAA AG ACA CTC CAG CTG GGT GTA ACA GCA ACT CC ACA CTC CAG CTG GGT AGG TAG TTT TAT GT ACA CTC CAG CTG GGC CCA GTG TTC AGA CT ACA CTC CAG CTG GGC CAG TGT TCA GAC TA ACA CTC CAG CTG GGT GTG CAA ATC TAT GC ACA CTC CAG CTG GGT GTG CAA ATC CAT GC ACA CTC CAG CTG GGT GGA ATG TAA AGA AG ACA CTC CAG CTG GGT AAC ACT GTC TGG TA ACA CTC CAG CTG GGT AAT ACT GCC TGG TA ACA CTC CAG CTG GGA GGG GCA TAG GGC AT ACA CTC CAG CTG GGG TGA AAT GTT TAG GA ACA CTC CAG CTG GGT TCC CTT TGT CAT CC ACA CTC CAG CTG GGT CCT TCA TTC CAC CG ACA CTC CAG CTG GGT GGA ATG TAA GGA AG ACA CTC CAG CTG GGA TAA GAC GAG CAT AA ACA CTC CAG CTG GGT AAA GTG CTT ATA GT ACA CTC CAG CTG GGC AAA GTG CTC ATA GT ACA CTC CAG CTG GGC TGT GCG TGT GAC AG ACA CTC CAG CTG GGT AAC AGT CTA CAG TC ACA CTC CAG CTG GGA CAG CAG GCA CAG AC ACA CTC CAG CTG GGT AGC TTA TCA GAC TG ACA CTC CAG CTG GGT AAT CTC AGC TGG CA ACA CTC CAG CTG GGA ACA GTA AGA GAT TA ACA CTC CAG CTG GGA AGG TCC AGC CTC AT ACA CTC CAG CTG GGA GCT ACA TTG TCT GC ACA CTC CAG CTG GGA GCT ACA TCT GGC TA ACA CTC CAG CTG GGT GTC AGT TTG TCA AA ACA CTC CAG CTG GGA AGC TGC CAG TTG AA ACA CTC CAG CTG GGA GTT CTT CAG TGG CA ACA CTC CAG CTG GGA TCA CAT TGC CAG GG ACA CTC CAG CTG GGG TGC CTA CTG AAC TG ACA CTC CAG CTG GGT GGC TCA GTT CAG CA ACA CTC CAG CTG GGC ATT GCA CTT GTC TC ACA CTC CAG CTG GGC CTA TTC TTG ATT AC ACA CTC CAG CTG GGT TCA AGT AAT CCA GG ACA CTC CAG CTG GGT TCA CAG TGG CTA AG ACA CTC CAg CTG GGG ACA GTC AGT AGT TG 
rsy-miR-29abc-3p

rsy-miR-29c-5p

rsy-miR-29d-3p

rsy-miR-301-3p

rsy-miR-30a-3p

rsy-miR-30a-5p

rsy-miR-30b-5p

rsy-miR-30d-5p

rsy-miR-30e-5p

rsy-miR-31b-5p

rsy-miR-320-3p

rsy-miR-338-3p

rsy-miR-33a-5p

rsy-miR-33b-5p

rsy-miR-34a-5p

rsy-miR-363-3p

rsy-miR-365-3p

rsy-miR-367-3p

rsy-miR-425-5p

rsy-miR-427-3p

rsy-miR-429-3p

rsy-miR-449a-5p

rsy-miR-449b-5p

rsy-miR-449c-3p

rsy-miR-451-5p

rsy-miR-455-5p

rsy-miR-7-5p

rsy-miR-92a-5p

rsy-miR-92b-3p

rsy-miR-93a-5p

rsy-miR-9406-3p

rsy-miR-9407-5p

rsy-miR-9-5p

rsy-miR-96-5p

rsy-miR-98-5p

rsy-miR-99/100-5p
ACA CTC CAG CTG GGT AGC ACC ATT TGA AA ACA CTC CAG CTG GGT GAC CGA TCT CTC TT ACA CTC CAG CTG GGT AGC ACC ATA TGA AA ACA CTC CAG CTG GGC AGT GCA ATA GTA TT ACA CTC CAG CTG GGC TTT CAG TCA GAT GT ACA CTC CAG CTG GGT GTA AAC ATC CTC GA ACA CTC CAG CTG GGT GTA AAC ATC CTA CA ACA CTC CAG CTG GGT GTA AAC ATC CCC GA ACA CTC CAG CTG GGT GTA AAC ATC CTT GA ACA CTC CAG CTG GGG GCA AGA TGC TGG CA ACA CTC CAG CTG GGA AAA GCT GGG TTG AG ACA CTC CAG CTG GGT CCA GCA TCA GTG AT ACA CTC CAG CTG GGG TGC ATT GTA GTT GC ACA CTC CAG CTG GGG TGC ATT GTT GTT GC ACA CTC CAG CTG GGT GGC AGT GTC TTA GC ACA CTC CAG CTG GGA ATT GCA CGG TAT CC ACA CTC CAG CTG GGT AAT GCC CCT AAA AA ACA CTC CAG CTG GGA ATT GCA CTG TAG CA ACA CTC CAG CTG GGA ATG ACA CGA TCA CT ACA CTC CAG CTG GGA AAG TGC TTT CTG TT ACA CTC CAG CTG GGT AAT ACT GTC TGG TA ACA CTC CAG CTG GGA GGC AGT GTA ATG TT ACA CTC CAG CTG GGA GGC AGT GTA GTT TT ACA CTC CAG CTG GGA GCT GCT AGC TGC AC ACA CTC CAG CTG GGA AAC CGT TAC CAT TA ACA CTC CAG CTG GGT ATG TGC CCT TGG AC ACA CTC CAG CTG GGT GGA AGA CTA GTG AT ACA CTC CAG CTG GGA GCA TTG CAA CCA AT ACA CTC CAG CTG GGT ATT GCA CTC GTC CC ACA CTC CAG CTG GGA AAG TGC TGT TCG TG ACA CTC CAG CTG GGA GCA AAT CTG TTG GT ACA CTC CAG CTG GGT GCG CTC GGT GGA TA ACA CTC CAG CTG GGT CTT TGG TTA TCT AG ACA CTC CAG CTG GGT TTG GCA CTA GCA CA ACA CTC CAG CTG GGT GAG GTA GTA AGT TG ACA CTC CAG CTG GGA ACC CGT AGA TCC GA

Snord 68
SnRNA U6
Universal Primer
Stem-loop Adapter

ACA CTC CAG CTG GGG CTG TAC TGA CTT G

ACA CTC CAG CTG GGG TGC TCG CTT CGG

CTC ACA GTA CGT TGG TAT CCT TGT G

CTC ACA GTA CGT TGG TAT CCT TGT GAT GTT CGA TGC CAT ATT GTA CTG TGA GTT TTT TTT TVN 
Table E2. Primers used for analysis of novel wood frog specific miRNAs in pooled $R$. sylvatica brain and liver, including miRNA-specific forward primers, universal reverse primer, and the stem-loop adapter for reverse transcription.

\begin{tabular}{|c|c|c|c|c|c|c|c|c|c|c|}
\hline \multirow{2}{*}{$\frac{\text { Target }}{\text { rsy-miR-novel1-3p }}$} & \multicolumn{10}{|c|}{ Primer Sequence (5'-3') } \\
\hline & $\overline{A C A}$ & CTC & CAG & CTG & GGA & AAG & TTC & TGA & GGG & $\mathrm{TA}$ \\
\hline rsy-miR-novel5-5p & $A C A$ & $\mathrm{CTC}$ & CAG & CTG & GGA & $\mathrm{CCC}$ & TGC & TCG & CAG & CG \\
\hline rsy-miR-novel6-5p & $A C A$ & $\mathrm{CTC}$ & CAG & CTG & GGA & GTA & $\mathrm{CCA}$ & $\mathrm{CAT}$ & TTC & TG \\
\hline rsy-miR-novel7-3p & $A C A$ & $\mathrm{CTC}$ & CAG & CTG & GGA & TAG & $\mathrm{CTC}$ & $\mathrm{TTT}$ & GAA & TG \\
\hline rsy-miR-novel8-3p & $A C A$ & $\mathrm{CTC}$ & CAG & CTG & GGA & TAT & $\mathrm{CCT}$ & TTG & $\mathrm{TCT}$ & $\mathrm{CT}$ \\
\hline rsy-miR-novel9-3p & $A C A$ & $\mathrm{CTC}$ & CAG & CTG & GGA & TAT & TGC & AGT & $\mathrm{CCA}$ & TG \\
\hline rsy-miR-novel10-3p & $A C A$ & $\mathrm{CTC}$ & CAG & CTG & GGA & TGA & $\mathrm{CAT}$ & $\mathrm{CTC}$ & $A A A$ & AT \\
\hline rsy-miR-novel12-5p & $A C A$ & $\mathrm{CTC}$ & CAG & CTG & GGA & TTG & AGG & CTA & $\mathrm{TCT}$ & GT \\
\hline rsy-miR-novel14-3p & $\mathrm{ACA}$ & $\mathrm{CTC}$ & CAG & CTG & GGA & $\mathrm{TTT}$ & GCG & TGC & TTA & $\mathrm{CT}$ \\
\hline rsy-miR-novel16/17-3p & $A C A$ & $\mathrm{CTC}$ & CAG & CTG & GGC & AGG & CTG & GTT & AGA & TG \\
\hline rsy-miR-novel18-5p & $A C A$ & $\mathrm{CTC}$ & CAG & CTG & GGC & CAC & CTC & $\mathrm{CCC}$ & TGC & $A A$ \\
\hline rsy-miR-novel19-3p & $A C A$ & $\mathrm{CTC}$ & CAG & CTG & GGC & $\mathrm{CTA}$ & CGG & $\mathrm{CCA}$ & TAC & $\mathrm{CA}$ \\
\hline rsy-miR-novel20-5p & $A C A$ & $\mathrm{CTC}$ & CAG & $\mathrm{CTG}$ & GGC & $\mathrm{CTC}$ & CAG & $\mathrm{CTG}$ & TTG & $\mathrm{CA}$ \\
\hline rsy-miR-novel21-5p & $A C A$ & $\mathrm{CTC}$ & CAG & CTG & GGC & $\mathrm{CTG}$ & $\mathrm{CAT}$ & TGT & $A C A$ & $\mathrm{CA}$ \\
\hline rsy-miR-novel22-3p & $A C A$ & $\mathrm{CTC}$ & CAG & CTG & GGC & $\mathrm{GTT}$ & GTA & GGC & $\mathrm{CTC}$ & $\mathrm{TC}$ \\
\hline rsy-miR-novel24-5p & $A C A$ & $\mathrm{CTC}$ & CAG & CTG & GGC & TGT & $\mathrm{ACA}$ & GAT & CAC & TG \\
\hline rsy-miR-novel25-5p & $A C A$ & $\mathrm{CTC}$ & CAG & CTG & GGG & $\mathrm{ACA}$ & GTC & $A G A$ & AGT & TG \\
\hline rsy-miR-novel26-5p & $A C A$ & $\mathrm{CTC}$ & CAG & CTG & GGG & AGG & $\mathrm{CTG}$ & $\mathrm{ATT}$ & $\mathrm{CTG}$ & AG \\
\hline rsy-miR-novel27-3p & $A C A$ & $\mathrm{CTC}$ & CAG & CTG & GGG & CGA & $\mathrm{CCC}$ & ATA & $\mathrm{CTT}$ & GG \\
\hline rsy-miR-novel29-3p & $A C A$ & $\mathrm{CTC}$ & CAG & CTG & GGG & CGG & ATA & TGG & GAC & $\mathrm{TG}$ \\
\hline rsy-miR-novel30-5p & $A C A$ & $\mathrm{CTC}$ & CAG & CTG & GGG & GGA & TGA & TGG & GAC & $\mathrm{TT}$ \\
\hline rsy-miR-novel32-3p & $A C A$ & $\mathrm{CTC}$ & CAG & CTG & GGG & TTG & TAG & GCC & $\mathrm{TCT}$ & $\mathrm{CC}$ \\
\hline rsy-miR-novel34-5p & $A C A$ & $\mathrm{CTC}$ & CAG & CTG & GGT & AGC & AGC & $A C A$ & CAG & $\mathrm{AT}$ \\
\hline rsy-miR-novel35-5p & $A C A$ & $\mathrm{CTC}$ & CAG & CTG & GGT & AGC & TTA & TCA & GAC & TG \\
\hline rsy-miR-novel36-3p & $A C A$ & $\mathrm{CTC}$ & CAG & CTG & GGT & AGT & GCA & $\mathrm{ATA}$ & TTG & $\mathrm{CT}$ \\
\hline rsy-miR-novel37-5p & $\mathrm{ACA}$ & $\mathrm{CTC}$ & CAG & CTG & GGT & $\mathrm{CAA}$ & TGA & $\mathrm{AAT}$ & CAA & $\mathrm{CC}$ \\
\hline rsy-miR-novel38-3p & $A C A$ & $\mathrm{CTC}$ & CAG & CTG & GGT & $\mathrm{CCT}$ & AGA & AGC & TTG & $\mathrm{AA}$ \\
\hline rsy-miR-novel39-5p & $A C A$ & $\mathrm{CTC}$ & CAG & CTG & GGT & $\mathrm{CCT}$ & $\mathrm{CAT}$ & TGT & $\mathrm{ACA}$ & TG \\
\hline rsy-miR-novel40-3p & $A C A$ & $\mathrm{CTC}$ & CAG & CTG & GGT & $\mathrm{CTT}$ & $\mathrm{TTT}$ & TGC & TGG & $\mathrm{AA}$ \\
\hline rsy-miR-novel41-3p & $A C A$ & $\mathrm{CTC}$ & CAG & CTG & GGT & GCA & GAT & $\mathrm{GCC}$ & TGT & GG \\
\hline rsy-miR-novel44-5p & $\mathrm{ACA}$ & $\mathrm{CTC}$ & CAG & CTG & GGT & GTA & $\mathrm{CCA}$ & TGC & TGG & TA \\
\hline rsy-miR-novel46-3p & $A C A$ & $\mathrm{CTC}$ & CAG & CTG & GGT & $\mathrm{TCA}$ & CTA & CTA & GCA & GA \\
\hline rsy-miR-novel47-5p & $\mathrm{ACA}$ & $\mathrm{CTC}$ & $\mathrm{CAG}$ & $\mathrm{CTG}$ & GGT & TGA & $\mathrm{CAT}$ & $\mathrm{CAT}$ & $\mathrm{CAT}$ & $\mathrm{AC}$ \\
\hline Universal Primer & CTC & $A C A$ & GTA & CGT & TGG & TAT & $\mathrm{CCT}$ & TGT & G & \\
\hline \multirow[t]{3}{*}{ Stem-loop Adapter } & $\mathrm{CTC}$ & $A C A$ & GTA & CGT & TGG & $\mathrm{TAT}$ & $\mathrm{CCT}$ & $\mathrm{TGT}$ & GAT & \\
\hline & GTT & CGA & TGC & CAT & $\mathrm{ATT}$ & GTA & $\mathrm{CTG}$ & $\mathrm{TGA}$ & GTT & \\
\hline & $\mathrm{TTT}$ & $\mathrm{TTT}$ & TVN & & & & & & & \\
\hline
\end{tabular}




\section{APPENDIX F}

Bioinformatics Workflow for TemperatureSensitive miRNA Target Prediction 


\section{OVERALL WORKFLOW:}

1. Install all programs and tools needed

2. Predict mRNA targets of significant miRNAs at ${ }^{\circ} \mathrm{C}$ of interest using FINDTAR3

3. Filter and sort through FindTar3 output

4. Convert Ensemble Transcript IDs from into gene symbols using BIODBNET

5. Map protein-protein interactions between predicted miRNA targets using STRING

6. Visualize protein-protein interactions and perform MCL clustering using CYTOSCAPE

7. Analyze the enriched functions of the clustered protein-protein interactions using $\mathbf{G O}$ ANALYSIS

\section{PROGRAMS AND ONLINE TOOLS:}

Programs to download and install:

- FindTar3: (http://bio.sz.tsinghua.edu.cn/)

- Cytoscape: (http://www.cytoscape.org/)

\section{Online bioinformatics tools and databases to use:}

- STRING:

(http://string-db.org/cgi/input.pl?Userld=vlwSrTrf48u1\&sessionld=b4pdpJEPgnju\&input page show search=on)

- Gene Ontology (GO) enrichment analysis: (http://www.geneontology.org/)

- bioDBnet - db2db: (https://biodbnet-abcc.ncifcrf.gov/db/db2db.php)

- miRBase: (http://www.mirbase.org/index.shtml)

- UCSC Genome Browser:

(http://genome.ucsc.edu/cgi-bin/hgTables?hgsid=585741047 yoc2Xw7DVSZdK52r2ZEcE9LOuC6Q)

*NOTE: Analyses below should be done in a Linux environment but some steps can be done in Windows

*NOTE: For more information on how these programs work- read their manuals and documentation

*NOTE: Run scripts from the correct directory and place the files you need in the appropriate directory 


\section{Predict mRNA gene targets for significantly changing miRNAs using FindTar3}

Once you have a list of significantly changing miRNAs you can use FindTar to predict their potential mRNA gene targets based on the 3'UTR regions. With FindTar you are also able to specify the temperature at which binding will be simulated, in this case a temperature of $-2^{\circ} \mathrm{C}$ was used for freezing as this is the frog's internal temperature after ice formation. [Linux only]

\section{Installing and running FindTar3}

- Download annotated 3'UTR regions of your reference animal. In this case, Xenopus tropicalis 3'UTRs were used

(http://genome.ucsc.edu/cgi-bin/hgTables?hgsid=585741047_yoc2Xw7DVSZdK52r2ZEcE9LOuC6Q)

- Download and install FindTar3 (http://bio.sz.tsinghua.edu.cn/)

- Read the FindTar manual and tutorial for option information

- Run the following script:

\$./findtar 1iver_nove1_frozen_down.fasta

Xenopus_3UTR.fasta -o Nove1_Liv_FZ_DOWN_5.txt -tab -

T 5 -at 5 -gt 2 -gc 5 -go -8 -ge -2 -score 20 energy -20

\section{FindTar output processing}

Next you must extract the 3'UTRs that were targeted by your significantly expressed miRNAs from your FindTar output. To do this:

- Organize the results on excel to remove all the Xenopus 3'UTRs that weren't predicted to be targeted by your group of miRNAs.

- Open the FindTar output in excel and use the following excel functions to clean up your sheet:

- Insert a column in A and number all the rows

- [column N] If cell underneath is empty = TRUE, full= FALSE $\rightarrow$ $=\operatorname{IF}(\operatorname{ISBLANK}(\mathrm{B} 3), 0,1)$

- $[$ column O] If cell has the word "xen" = TRUE $\rightarrow$ $=$ ISNUMBER (SEARCH("xen", B2))

- [column P] If $\mathrm{N}=0$ and $\mathrm{O}=\mathrm{TRUE}$ then the function is true $\rightarrow$ $=$ AND $(N 2=0,02=1)$

- [column Q] If $\mathrm{O}=$ TRUE and $\mathrm{P}=\mathrm{TRUE}$, then DELETE $\rightarrow$ $=\operatorname{IF}(\mathrm{P} 2=1$, "DELETE" , " ")

- [column R] If cell is blank =delete blank $\rightarrow=\operatorname{IF}(\operatorname{ISBLANK}(B 2)$, "DELETE BLANK", " ") 
- Copy columns $Q$ and $R$ into columns $S$ and T (TEXT ONLY), respectively.

- Organize the sheet by columns $S$ and $T$ and then delete all the rows that have 'DELETE' in column $S$ and 'DELETE BLANK' in column T.

- Below is a screenshot of how the columns and functions should be used:

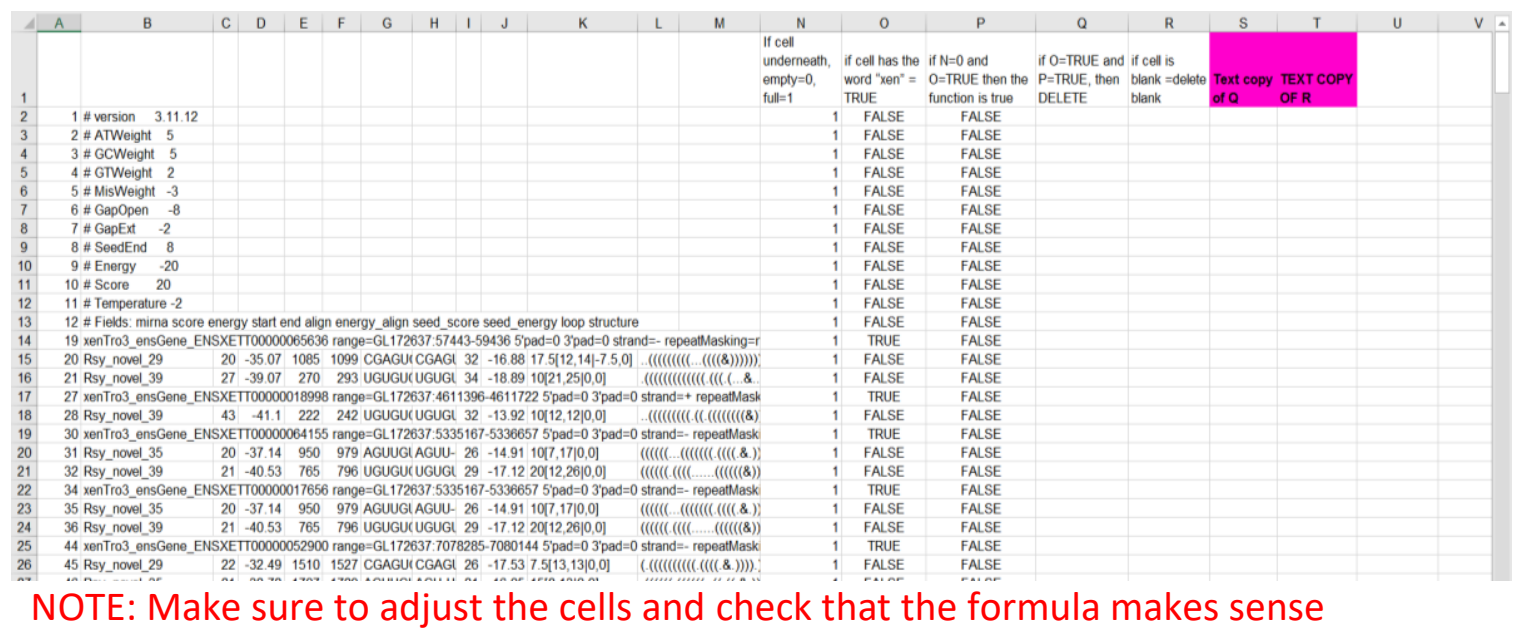

To isolate the Ensemble transcript IDs in a separate column you need to open the cleaned up excel file (with just the targeted mRNAs) in a text editor:

- In this case, this was the 3'UTR identifier format:

xenTro3_ensGene_ENSXETT00000065636

range $=$ GL172637:57443-59436 5 ' pad=0 3 'pad=0 strand=repeatMasking=none

- Replace "xenTro3_ensGene_" and "range=" with a 'tab'

- Save this file and open it with excel

- Now all the ensemble transcript IDs will be in a separate column, ready for the next step

\section{Convert Ensemble transcript IDs to gene symbols}

[Linux and Windows friendly]

- Open the $\mathrm{db} 2 \mathrm{db}$ tool hosted on bioDBnet: (https://biodbnet-abcc.ncifcrf.gov/db/db2db.php)

- Input: 'Ensemble transcript ID'

- Outputs: 'Gene symbol'

- Copy the ensemble transcript ID list you made in the previous section, example:

ENSXETT00000047050

ENSXETT00000018929

- Click 'Submit' $\rightarrow$ Click the 'Results in excel' button to download the table

- Open the file in excel and when opening the file separate the columns by 'tab' and 'semicolon' 
- For transcript IDs with multiple gene symbols- you can delete all the other names as they are all synonyms and only one name is needed. Keep the name in the main column and delete the other columns.

\section{Mapping protein-protein interactions}

The list of targeted genes will be run through the STRING database to identify all predicted and experimentally validated protein-protein interactions.

[Linux and Windows friendly]

- Open the STRING 'Search' page:

(http://string-

db.org/cgi/input.pl?Userld=vlwSrTrf48u1\&sessionld=b4pdpJEPgnju\&input page active form=multipl e identifiers)

- Select the 'Multiple proteins' option

- Copy your list of gene symbols into 'List of names' box

- Select your organism of interest, in this case $X$. tropicalis was selected $\rightarrow$ Click 'Search'

- A webpage with the gene names and the proteins they encode will open $\rightarrow$ Click 'Continue'

- This will generate a protein interaction network, Here is an example of a large network:

- Click 'Table/Exports' and download the network as 'as simple tabular text output'

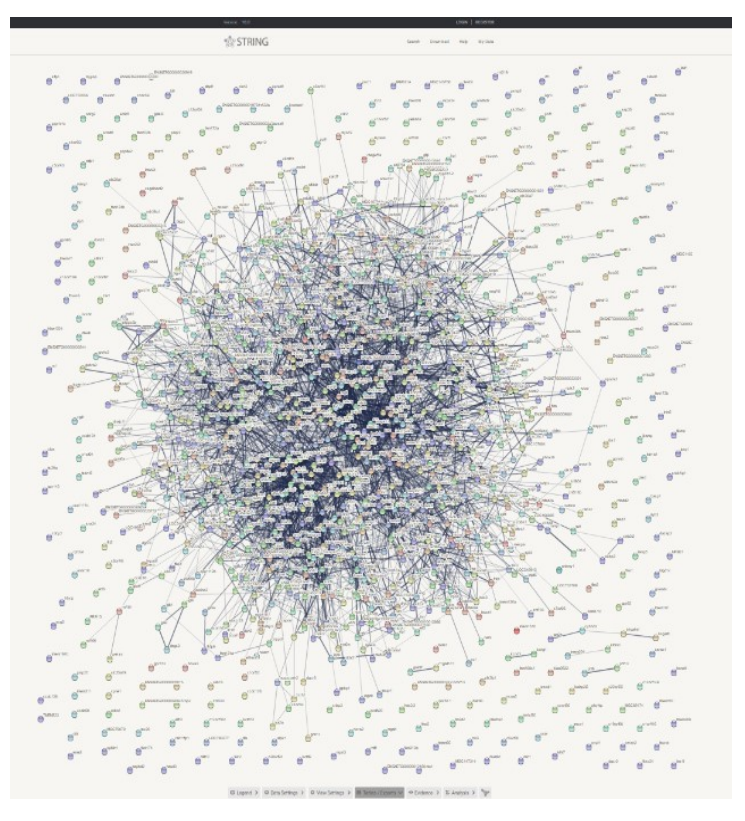

\section{Visualizing protein networks and clustering}

You can now perform cluster analysis on the protein-protein networks you have generated to identify potential binding partners and functional interactions.

[Linux and Windows friendly]

- Download and install Cytoscape (http://www.cytoscape.org/)

- Create a new network and give it an appropriate name, ex (Rsy_Liver_Frozen_Up)

- Click the 'Import network' button and select the string interaction network for Rsy_Liver_Frozen_Up

- Generate a cluster using 'clustrmaker' $\rightarrow$ Select 'MCL clustering' $\rightarrow$ Select 'combined scores' 
- You should also play around with the style and visual appearance of your clustered network.

\section{Enriching for biological functions in protein clusters}

$\mathrm{GO}$ analysis can be used to determine if any of the protein clusters generated are enriched for a particular biological process or cellular function.

[Linux and Windows friendly]

- On Cytoscape, open up the 'node table' $\rightarrow$ organize the table by MCL cluster \#

- Select a cluster (e.g. cluster 1 ) and highlight the names of all proteins from that cluster

- Copy this list into the GO analysis box (http://www.geneontology.org/)

- Select 'biological processes' (you can also play with molecular function and cellular component)

- Select 'Xenopus tropicalis'. Or select the organism that is the most evolutionarily similar to your animal of study.

- Analyze the enriched network output. Organize the biological processes by 'hierarchical view' or 'fold change'. Only discuss the process found to be significantly enriched with a large fold difference. 


\section{APPENDIX G}

Bioinformatics Workflow for Discovery of Novel Species-Specific MicroRNA 


\section{OVERALL WORKFLOW:}

1. Install all programs and tools needed

2. Obtain small RNA-Seq FASTQ files, cleaned and trimmed

3. Convert FASTQ files into FASTA files using BLASTSTATION or GALAXY

4. Identify conserved miRNAs in wood frog RNA-Seq using NCBI BLAST+ and MIRBASE

5. Pre-process files for novel miRNA discovery using GALAXY and PYFASTA

6. Use MIRDEEP2 to identify novel microRNAs

7. Filter and sort through miRDeep2 output

8. Check that the predicted novels do not exist on MIRBASE using BLAST+

9. Validate the identity and sequences of the predicted novels with RT-qPCR

10. Quantify, normalize, and graph RT-qPCR data of novel miRNAs using RBIOPLOT

11. Refer to APPENDIX $\boldsymbol{F}$ for the bioinformatics workflow on predicting mRNA targets of significantly changing miRNAs, at ${ }^{\circ} \mathrm{C}$ of interest, using FINDTAR3 + BIODBNET + STRING + CYTOSCAPE + GO ANALYSIS

\section{PROGRAMS AND ONLINE TOOLS:}

\section{Programs to download and install:}

- miRDeep2 (https://www.mdc-berlin.de/8551903/en/)

- Pyfasta (https://pypi.python.org/pypi/pyfasta/)

- BLAST+ (https://blast.ncbi.nlm.nih.gov/Blast.cgi?PAGE TYPE=BlastDocs\&DOC TYPE=Download)

- FASTQC (https://www.bioinformatics.babraham.ac.uk/projects/fastac/)

- Fastq-mcf (https://expressionanalysis.github.io/ea-utils/)

\section{Online bioinformatics tools and databases to use:}

- Bugaco Sequence converter:

(http://sequenceconversion.bugaco.com/converter/biology/sequences/tab to fasta.php)

- Galaxy: (https://usegalaxy.org/)

- bioDBnet - db2db (https://biodbnet-abcc.ncifcrf.gov/db/db2db.php)

- miRBase (http://www.mirbase.org/index.shtml)

- UCSC Genome Browser:

(http://genome.ucsc.edu/cgi-bin/hgTables?hgsid=585741047_yoc2Xw7DVsZdK52r2ZEcE9LOuC60)

*NOTE: Analyses below should be done in a Linux environment but some steps can be done in Windows

*NOTE: For more information on how these programs work- read their manuals and documentation

*NOTE: Run scripts from the correct directory and place the files you need in the appropriate directory 


\section{SMALL RNA-SEQUENCING [INPUT]:}

For this analysis, I used wood frog small RNA-sequencing files (FASTQ). Sequencing was performed on an Illumina platform (Genome Quebec). The files we obtained were trimmed of adapters and cleaned of low quality reads. This analysis was based on three wood frog sequencing runs:
1) Frozen liver
2) Anoxic liver
3) Anoxic heart

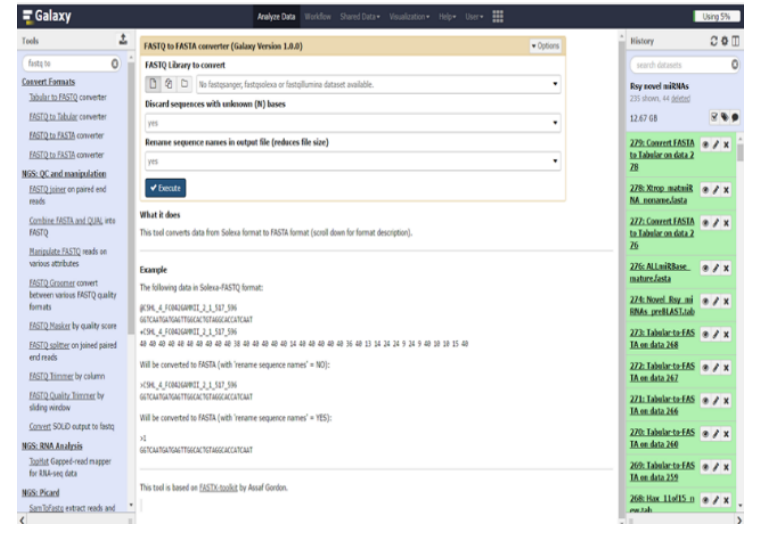

\section{METHODS:}

RNA-Seq raw read pre-processing [OPTIONAL]

If the sequencing facility sends raw Illumina reads then you must first remove all lowquality reads and trim the sequencing adapters off your reads.

[Linux only]

1) Quality control-

- Download and install FASTQC (https://www.bioinformatics.babraham.ac.uk/projects/fastqc/)

- Perform quality checks on FASTQ files using FASTQC

- Read and follow the FASTQC manual

- Possible criteria to include in the script

- Remove reads $<16$ or $18 \mathrm{nt}$ and $>60 \mathrm{nt}$

- Remove reads without insert fragment between adapters

- Remove reads containing poly $(A)$ stretches

- Remove poor quality reads, such as reads with a Q Score $<20$

\section{2) Adapter trimming-}

- Download and install Fastq-mcf (https://expressionanalysis.github.io/ea-utils/)

- Read and follow the fastq-mcf manual

\section{Converting FASTQ to FASTA}

Next you need to convert the FASTQ files into FASTA files as we need FASTA files for the subsequent blasting and discovery steps.

[Linux and Windows friendly]

- Open the Galaxy server (https://usegalaxy.org/)

- Create a free account, allows you to save up to $250 \mathrm{~Gb}$ on the Galaxy cloud server

- Upload your FASTA files of interest

- Use their 'FASTQ to FASTA converter' 
- Download the newly converter FASTQ file

- For additional instructions visit (https://galaxyproject.org/support/)

\section{BLASTing for conserved miRNAs}

The NCBI BLAST+ tool can be used to blast your query small RNA-Seq reads against databases of known precursor and mature miRNAs, this will identify conserved miRNAs in your query file(s).

[Linux and Windows friendly]

Downloading and installing BLAST+

- Download and install NCBI BLAST+

(https://blast.ncbi.nlm.nih.gov/Blast.cgi?PAGE TYPE=BlastDocs\&DOC TYPE=Download)

- You should save this file to the $\mathrm{C}: / /$ drive as this will make it easy to access from the terminal (Linux) or command prompt (Windows). The below manuals are SUPER useful, read them for more detailed instructions.

- Quick Start BLAST+ user manual

(https://www.ncbi.nlm.nih.gov/books/NBK279680/)

- Options for BLAST+ command-line applications

(https://www.ncbi.nlm.nih.gov/books/NBK279675/)

- Full BLAST+ user manual

(https://www.ncbi.nlm.nih.gov/books/NBK279690/)

\section{Building a local BLAST+ database}

Before you can search you should BLAST your query small RNA-seq FASTA files, you need to convert the files into a local database to allow the program to organize, parcel, and retrieve your reads. This local database will be our query database.

- Follow these instructions to build a local database

(https://www.ncbi.nlm.nih.gov/books/NBK279688/)

- Change into the BLAST+ 'bin' directory

- Copy your query FASTA files into this bin directory

- To build a database run a variation of the BLAST+ makedb command \$ makeblastdb -in HI.2163.008.RPI20.HRT_Anox.fasta parse_seqids -dbtype nucl

- To customize this command, replace HI . 2163 .008. RPI20.HRT_Anox . fasta with the name of the query FASTA file you want to convert into a database.

- This command requires a lot of computing power and can take over an hour, depending on the size of your query FASTA files. Don't lose hope. 
NOTE: If you copy any scripts from .doc or .txt into the command prompt then you should re-write the hyphen (-), because the command prompt uses a special hyphen character

\section{Create libraries of precursor and mature miRNA reference sequences from miRBase}

If you are working on an animal with known miRNAs that have been annotated and deposited to miRbase, then you should download that animals miRNA library. If you are working on a non-genome sequenced animal or on an animal with no miRNAs deposited on miRbase then you should use precursor and mature miRNA sequence from evolutionarily similar animals. In this case, because I am working with the non-sequenced wood frog, I constructed miRNA precursor and mature libraries using:

- Frog $\rightarrow$ Xenopus laevis

- Frog $\rightarrow$ Xenopus tropicalis

- Chicken $\rightarrow$ Gallus gallus

- Lizard $\rightarrow$ Anolis carolinensis

To download miRNAs of a particular animal:

- Open miRBase (http://www.mirbase.org/index.shtml)

- Click the 'Browse' button in the menu bar $\rightarrow$ Click 'Expand all' $\rightarrow$ Find your animal(s) of interest

- Scroll to the bottom of the page $\rightarrow$ Select 'Stem-loop sequence' for precursors OR 'mature sequences' for mature miRNAs $\rightarrow$ Select 'Unaligned fasta format' $\rightarrow$ Click the 'Select all' button

- Select 'Fetch Sequences' and copy all sequences into a text file and save it

- File name and content (Xtrop_matmiRNA.fasta)

>xtr-miR-202-5p MIMAT0003620

UUUCCUAUGCAUAUACCUCUUU

>xtr-miR-200a MIMAT0003693

UAACACUGUCUGGUAACGAUGU

- Format the identifiers to look like the example below using the 'Replace all' function in your text editor. You need to do this to remove the hyphens, whitespaces, and miRBase accession numbers.

$>x t r \_m i R \_202 \_5 p$

UUUCCUAUGCAUAUACCUCUUU

$>$ xtr_miR_200a

UAACACUGUCUGGUAACGAUGU 


\section{Searching your query database against conserved reference miRNAs}

Now, you can BLAST your database against your miRBase constructed libraries of conserved mature miRNA sequences. To BLAST databases run the 'blastn' command.

- For instructions on how to customize the different options refer to the NCBI manual

- To blast databases you need to run this script:

\$ blastn -db HI.2163.008.RPI20.HRT_Anox.fasta -query

X_laevis_premiRNA.fasta -out HRT_AX_X1av_premiRNA.out

-num_alignments 1 -num_threads 20 -outfmt 6

- In this example, I blasted my Rsy anoxic heart database (HI.2163.008.RPI20.HRT_Anox.fasta) against known X. laevis mature miRNAs (X_laevis_premiRNA.fasta). This generates the tabular file (HRT_AX_X1av_premi RNA. out).

- Below is an excerpt from the HRT_AX_X1av_premi RNA. out file:

\begin{tabular}{|c|c|c|c|c|c|c|c|c|c|c|c|c|c|c|c|}
\hline$\angle$ & A & B & C & D & E & $\mathrm{F}$ & & G & $\mathrm{H}$ & 1 & J & K & L & M & $\mathrm{N}$ \\
\hline 1 & QUERY & Target label & $\begin{array}{l}\% \\
\text { identity }\end{array}$ & $\begin{array}{l}\text { Alignment } \\
\text { length }\end{array}$ & $\begin{array}{l}\# \text { of } \\
\text { mismatches }\end{array}$ & $\begin{array}{l}\text { \# gap } \\
\text { opens }\end{array}$ & & $\begin{array}{l}\text { START in } \\
\text { query }\end{array}$ & $\begin{array}{l}\text { END in } \\
\text { query }\end{array}$ & $\begin{array}{l}\text { START in } \\
\text { target }\end{array}$ & $\begin{array}{l}\text { End in } \\
\text { target }\end{array}$ & e-value & Bit score & & \\
\hline 2 & xla-mir-15c & D69F08P1:374:C56KDACXX:8:22 & $2 \quad 100$ & 47 & 0 & & 0 & 27 & 73 & 1 & 47 & $7.00 \mathrm{E}-17$ & 87.9 & & \\
\hline 3 & xla-mir-92a-1 & D69F08P1:374:C56KDACXX:8:22 & 100 & 28 & 0 & & 0 & 49 & 76 & 1 & 28 & $3.00 \mathrm{E}-06$ & 52.8 & & \\
\hline 4 & xla-mir-133a & D69F08P1:374:C56KDACXX:8:23 & 100 & 28 & 0 & & 0 & 53 & 80 & 1 & 28 & $3.00 \mathrm{E}-06$ & 52.8 & & \\
\hline 5 & xla-mir-142 & D69F08P1:374:C56KDACXX:8:23 & 97.44 & 39 & 1 & & 0 & 14 & 52 & 1 & 39 & $1.00 \mathrm{E}-10$ & 67.6 & & \\
\hline 6 & xla-mir-703 & D69F08P1:374:C56KDACXX:8:23 & 89.13 & 46 & 0 & & 4 & 20 & 60 & 1 & 46 & 4.00E-06 & 52.8 & & \\
\hline
\end{tabular}

If the file is columns are not labelled go to (http://www.drive5.com/usearch/manual/blast6out.html) for the proper column headers.

NOTE: Sometimes you will not find any matches with the mature miRNA sequences

Since the matches were found in the precursors you need to check that your RNA-seq read matches with the precursor's miRNA coding region. To do this:

- Go to miRBase $\rightarrow$ 'Search' from the menu bar $\rightarrow$ input name of miRNA and species in the search box $\rightarrow$ check, for example, that the xla-mir-133a region between nucleotide 53-80 harbors either the 5' or 3' miRNA sequences

\section{Finding and validating conserved miRNAs}

Now that we know which reads and which regions match with conserved miRNA sequences we can use the 'blastcmd' to extract the actual wood frog sequence that matched.

- For information on blastcmd options read the BLAST+ manual

- Run the Blastcmd command:

\$ blastdbcmd -db HI.2163.008.RPI16.LIV_Frozen.fasta dbtype nuc 1 
D69F08P1:374:C56KDACXX:8:1204:2079:83550 -range 1-50 outfmt \% $f$

- Because you need to do this separately for each conserved precursor/mature miRNA, it will be easier to combine all the scripts for each individual miRNA on excel and then you can convert this into a batch file by changing the file extension to (.bat). The excel file with all the blastcmd scripts will look like this, where only column $\mathbf{G}$ and column I are unique to the separate miRNAs:

\begin{tabular}{|c|c|c|c|c|c|c|c|c|c|c|c|}
\hline 4 & A & B & C & D & E & $\mathrm{F}$ & G & H & I & J & K \\
\hline 1 & blastdbcmd & $-\mathrm{db}$ & HI.2163.008.RPI16.LIV_Frozen.fasta & -dbtype & nucl & -entry & D69F08P1:374:C56KDACXX:8:1204:2079:83550 & -range & $1-50$ & -outfmt & $\% \% f$ \\
\hline 2 & blastdbcmd & $-d b$ & HI.2163.008.RPI16.LIV_Frozen.fasta & -dbtype & nucl & -entry & D69F08P1:374:C56KDACXX:8:1315:20357:76364 & -range & $1-29$ & -outfmt & $\% \% f$ \\
\hline 3 & blastdbcmd & $-d b$ & HI.2163.008.RPI16.LIV_Frozen.fasta & -dbtype & nucl & -entry & D69F08P1:374:C56KDACXX:8:1314:20035:56892 & -range & $2-50$ & -outfmt & $\% \% f$ \\
\hline 4 & blastdbcmd & $-d b$ & HI.2163.008.RPI16.LIV_Frozen.fasta & -dbtype & nucl & -entry & D69F08P1:374:C56KDACXX:8:2214:4626:11552 & -range & $1-50$ & -outfmt & $\% \% f$ \\
\hline 5 & blastdbcmd & $-\mathrm{db}$ & HI.2163.008.RPI16.LIV_Frozen.fasta & -dbtype & nucl & -entry & D69F08P1:374:C56KDACXX:8:2304:10438:89728 & -range & $1-28$ & -outfmt & $\% \% f$ \\
\hline
\end{tabular}

NOTE: You need to use two '\%' in column $\mathbf{k}$ symbols in your batch file because when you convert a .txt file to a batch file a single '\%' will be deleted but if you use 2 then one will be deleted and the second one will remain and your command will run properly.

To convert the above excel file into a batch file:

- Run the 'Replace all' function to remove the tabs (replace 'tab' with 'space').

NOTE: Copy paste the tab into the box, if you press the tab button in the box, it will not work as this will appear as a space not a tab.

- Change the file from a text file to an executable batch file $\rightarrow$ Change file extension from '.txt' to '.bat'.

- If you can't see the file names and you can't rename the extension follow this link and it should enable this feature: http://kb.winzip.com/kb/entry/26/

\section{Predicting novel miRNAs using miRDeep2}

miRDeep2 is a tool that can identify known and novel miRNAs by analyzing RNAsequencing data.

[Linux only]

\section{Installation and setup}

- Download and install miRDeep2 (https://www.mdc-berlin.de/8551903/en/)

- Once installed, you will see a tutorial directory. Copy its contents into the main mirdeep directory

- Run through the tutorial file to make sure that everything is working properly

- For detailed information on mirdeep functions and options read the tutorial and help file 


\section{Input file preprocessing}

To use miRDeep you need ensure that your input RNA-Seq reads have identifiers that match the miRDeep format. Below is the mirdeep error you get if your input file headers do not comply with the mirdeep format:

has to have the format

name_uniqueNumber_xnumber

- The below scripts were used to change the identifier names. Here is a sample entry from my input RNA-seq fasta file:

>D69F08P1: 374:C56KDACXX: 7:1101:1973:2190

NAACTATGATGAGTCTATCCCGAACCTGAATTGGTGATGACACACTTTTA

(1) This script was used to replace "D69F08P1:374:C56KDACXX:7:" with "LFr"

$\$$ per1 - $i$-pe 's/DD69F08P1:374:C56KDACXX:7:\b/LFr_/g' test3333. fasta

(2) This was used to remove all ":"

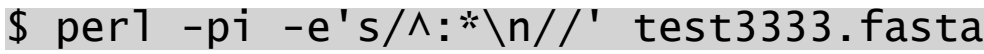

(3) Below used to save this to a new file

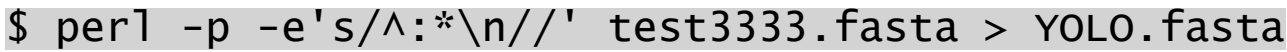

(4) To add text to the start of a header and save that to a new file

$\$$ perl $-p-e \quad " s / \wedge>/>L A x \_/ g "$ test_new.fasta > test_new2.fasta

(5) To add text to the end of a header and then overwrite your new file

$\$$ per1 -p -e ' $s / \wedge\left(>{ }^{*}\right) \$ / \$ 1 \_x 1 / g^{\prime}$ test_new2.fasta > test_new3.fasta

(6) Open this file in a text editor and use the 'replace all' function to remove the large space between the unique number and the "_x1"

NOTE: When changing the fundamental features of important files you should always keep a copy of your original file and save all changes to a new file.

[OPTIONAL]

Depending on your file size, you might have to parse your large FASTA files into smaller ones to be able to change all the identifiers into the mirdeep format using excel and galaxy. To do this you can use pyfasta.

- Download and install pyfasta (https://pypi.python.org/pypi/pyfasta/)

- To split fasta files run:

\$ split -n 15 Liv_anox_reads_3.fasta 
- To combine multiple fasta files back into ONE BIG FASTA FILE $\rightarrow$ Make a new folder $\rightarrow$ move all fasta files you wish to combine into this new directory $\rightarrow$ change into this directory $\rightarrow$ run the below script

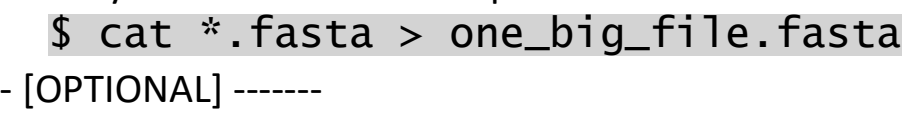

- You might also need to convert your file between tabular and fasta (and vice versa) to fix any other issues you might have with your identifier and the miRDeep format.

- Once identifier changes are completed, run a sanity check on your file to ensure it is in the proper format. See 'Read me' manual.

\section{\$ sanity_check_reads_ready_file.pl yourfile.fasta}

\section{Reference file compilation}

Now you need to prepare all your reference files... :O After preparing the below files, make sure to run a sanity check on each of them to ensure it is in the proper format. See 'Read me' manual.

(1) Reference genome (.fasta). In this case, the $X$. tropicalis genome was downloaded from UCSC Genome Browser (http://genome.ucsc.edu/cgibin/hgTables?hgsid=585741047 yoc2Xw7DVSZdK52r2ZEcE9LOuC6Q). [File name- xenTro7.fa]. Run a sanity check:

\section{\$ sanity_check_genome.pl}

(2) Reference miRBase mature miRNAs for the species (.fasta). $X$. tropicalis mature miRNAs were used. This file must also have identifiers that are compatible with mirdeep. [File name-Xtrop_premiRNAs_noname.fasta]

- Fasta file was opened in a text editor and all whitespaces and hyphens were removed

- This was saved and opened in .tabular

- Columns B, C, and D were added and filled accordingly $(\mathrm{xmi}=$ xenopus mature miRNAs; column $B$ is miRNA

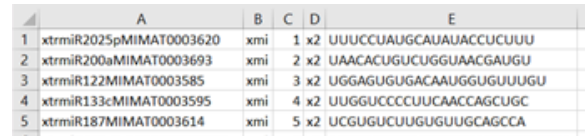
number; column $C$ has to be an ' $x$ ' and a single number). Example attached: - This was saved and then opened in a text editor and 'tabs' were removed to make file resemble a proper fasta file. See below:

$>$ xmi_1_x2

UUUCCUAUGCAUAUACCUCUUU

$>$ xmi_2_x2

UAACACUGUCUGGUAACGAUGU

$>x$ mi_3_x2

UGGAGUGUGACAAUGGUGUUUGU 
- Make sure to keep the original list so you can go back to it and figure out what miRNA corresponds to 'xmi_3_x2'

- Run a sanity check:

\$ sanity_check_mature_ref.pl

(3) Reference miRBase mature miRNAs for related species (.fasta). In this example, the same related animals used for BLASTing were used (Chicken, lizard, Xenopus laevis). This file was processed similarly to the xenopus mature miRNAs described above. [File name- other_matmiRNAs_noname.fasta].

(4) Reference miRBase precursor miRNAs for the species (.fasta). This file was processed similarly to the xenopus mature miRNAs. [File nameXtrop_premiRNAs_noname.fasta]

\section{Mapping reads to genome}

Run the below command

$\begin{array}{lllllll}\text { mapper.p1 Liv_anox_reads_3.fasta } & -\mathrm{c} & -\mathrm{j} & -1 & 18 & -\mathrm{m} & -\mathrm{p} \\ \text { xenTro7 } & -\mathrm{s} & \text { Liv_anox_reads_collapsed.fasta } & & -\mathrm{t} \\ \text { Liv_anox_reads_collapsed_vs_genome.arf } & -\mathrm{v}\end{array}$

\section{Finding novels!!}

Before running the mirdeep2 function you need to find the proper name of the species to allow mirdeep to map back to the UCSC genome browser. Run the following script, and find the name of your genome species from the list. In this case, it was 'X.tropicalis2':

$\$$ miRDeep2.p1 -u

Now you are ready to run the mirdeep 2 command and find novels!

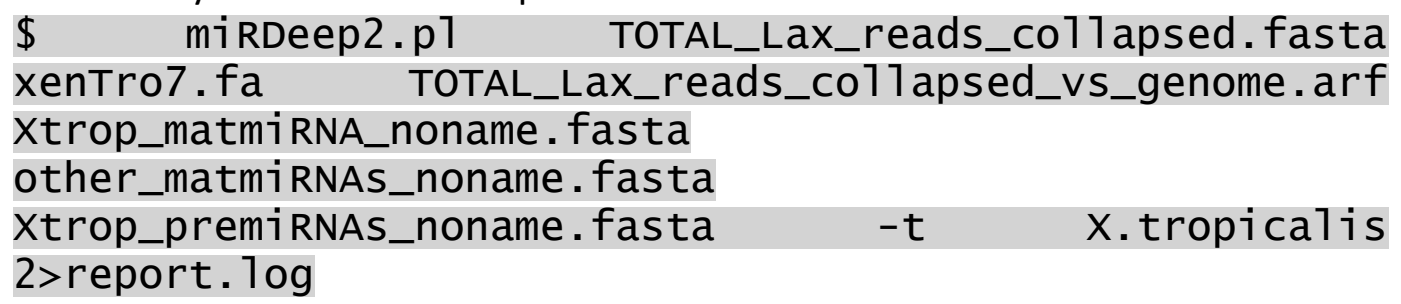

\section{Processing list of novels}

You will get a list of conserved miRNAs and a list of novel miRNAs. You can go through the conserved miRNA list and extract both the precursor and mature miRNAs- make sure to remove any duplicates. To process he novels:

- Open the miRDeep2 output in excel and organize the sequences by mature miRNA sequence.

- Go through the list and remove any redundant mature and precursor miRNA sequences. 
- Only keep potential novels that have unique mature and/or precursor sequences

\section{Verify that the novel miRNAs are ACTUALLY novel}

Now that you have a list of predicted novel miRNAs, you need to ensure that none of them match sequences for existing known miRNAs.

[Linux and Windows friendly]

- Download all the mature miRNAs on miRBase in FASTA format.

- Remove all whitespaces and hyphens

- Build a local database with your FASTA list of predicted novel miRNAs (See section 5.3.2.)

- Blast your novels against the list of all known miRBase miRNAs using the blastn command

$$
\$ \text { blastn -db ALLmiRBase_mature.fasta -query }
$$
Nove1_RSy_miRNAS_preBLAST.fasta -out results.out num_alignments 1 -num_threads 20 -outfmt 6

- Nothing should match in the blastn $\rightarrow$ TA DA!! NOVEL!!!

- If any of the novels happen to match existing miRNAs then remove them from your list of predicted novel miRNAs

\section{Validate candidate novel miRNAs with RT-qPCR}

For information on miRNA RT-qPCR refer to (http://www.nature.com/articles/srep24627)

- Only novel miRNAs with a single tight melt curve peak that also had low errors were kept as the novel miRNAs. Predicted novels with bad melt curves and high errors were discarded.

- Make a FASTA file with the names and sequences of the novel miRNAs that were found to significantly change in each of the conditions.

- Example file $\rightarrow$ Novel miRNAs significantly upregulated in frozen frog livers $>$ Rsy_novel_14

auuugcgugcuuacuuccca

>Rsy_novel_29

gcggauaugggacugagc

$>$ Rsy_novel_35

uagcuuaucagacugauguuga 


\section{APPENDIX H}

Novel Wood Frog Specific MicroRNA Sequences 
Table H1. Annotated precursor and mature novel microRNA sequences.

\begin{tabular}{|c|c|c|c|}
\hline MicroRNA & Mature sequence & Precursor sequence & Precursor coordinates \\
\hline rsy-miR-novel1-3p & aaaguucugaggguaccu & $\begin{array}{l}\text { gucucaaaaaugucagacuungaauaauuaua } \\
\text { aaguucugaggguaccu }\end{array}$ & KB021649:17309213..17309262:- \\
\hline rsy-miR-novel5-5p & acccugcucgcagcgcca & $\begin{array}{l}\text { acccugcucgcagcgccaaauuguguaaggcc } \\
\text { uauuncaccaggcacucaguuungguugcugu } \\
\text { uugucuugcca }\end{array}$ & KB021649:185517653..185517728:- \\
\hline rsy-miR-novel6-5p & aguaccacauuucugaugcug & $\begin{array}{l}\text { aguaccacauuucugaugcugaaaugcuacau } \\
\text { ccaaaaaugcagcaucaggauggguggcaccu } \\
\text { c }\end{array}$ & KB021651:13718358..13718423:+ \\
\hline rsy-miR-novel7-3p & auagcucuungaaugguacu & $\begin{array}{l}\text { cgccauuunccgagcuanaaacaguaucauug } \\
\text { ucauagcucuungaaugguacu }\end{array}$ & KB021651:72902570..72902624:+ \\
\hline rsy-miR-novel8-3p & auauccuungucucugag & $\begin{array}{l}\text { cagaggguauuuauguacuauguauaucugua } \\
\text { uauccuungucucugag }\end{array}$ & KB021657:51395541..51395590:+ \\
\hline rsy-miR-novel9-3p & auauugcaguccauguug & $\begin{array}{l}\text { aaguggcucgcaagccaauauauugcagucca } \\
\text { uguug }\end{array}$ & KB021658:105607327..105607364:+ \\
\hline rsy-miR-novel10-3p & augacaucucaaaaugagcggag & $\begin{array}{l}\text { ccacccauuungggacgucauuguggcaccua } \\
\text { cgcauuccaaugacaucucaaaaugagcggag }\end{array}$ & KB021650:27225935..27225999:- \\
\hline rsy-miR-novel12-5p & auugaggcuaucuguguagc & $\begin{array}{l}\text { aungaggcuaucuguguagccugcucuuauga } \\
\text { cсcggcuacccauguaacccuuuuaca }\end{array}$ & KB021656:86807609..86807668:- \\
\hline rsy-miR-novel14-3p & auuugcgugcuuacuuccca & $\begin{array}{l}\text { aggaggcaugcccucaaauccuguuaacaaau } \\
\text { aacaggauungcgugcuuacuuccca }\end{array}$ & KB021657:85902793..85902851:+ \\
\hline rsy-miR-novel16-3p & caggcugguuagaugguuau & $\begin{array}{l}\text { agcaggcaucuucucagccuacauguagauug } \\
\text { ucaaaucugcaggcuggunagaugguuguca }\end{array}$ & KB021654:83538137..83538200:+ \\
\hline rsy-miR-novel18-5p & ccaccuccccugcaaacgucc & $\begin{array}{l}\text { ccaccuccccugcaaacguccagugacgcaga } \\
\text { gguaauggacguuggcucugguggugaug }\end{array}$ & KB021649:52590231..52590292:- \\
\hline rsy-miR-novel19-3p & ccuacggccauaccacccu & $\begin{array}{l}\text { gaguagaagcgccuaggagauguuuaagagga } \\
\text { cgaaaagucagcuuagucaucaccuacggcca } \\
\text { uaccacccu }\end{array}$ & KB023293:7552..7625:- \\
\hline
\end{tabular}


rsy-miR-novel20-5p

ccuccagcuguugcagaacua

rsy-miR-novel21-5p ccugcauuguacacacugugu

rsy-miR-novel22-3p cguuguaggccucuccagcauu

rsy-miR-novel24-5p cuguacagaucacugcguugc

rsy-miR-novel25-5p gacagucagaaguuggucugg

rsy-miR-novel26-5p gaggcugauucugagcaa

rsy-miR-novel27-3p gcgacccauacuugguuuca

rsy-miR-novel29-3p gcggauaugggacugagc

rsy-miR-novel30-5p gggaugaugggacuuguaguc

rsy-miR-novel32-3p guuguaggccucuccagcauu

rsy-miR-novel34-5p uagcagcacacagauauuggca

rsy-miR-novel35-5p uagcuuaucagacugauguuga

rsy-miR-novel36-3p uagugcaauauugcuuauaggguc u

rsy-miR-novel37-5p ucaaugaaaucaaccuauccugu ccuccagcuguugcagaacuacaugcacccug uugcuuaunguuguucugugacagcuggaggg c

ccugcaunguacacacugugugugcaccucag acuugcacagcgcauacaauguggaug

ugcuggaguggacaucaaugcaunauuugca aggcguuguaggccucuccagcauu

cuguacagaucacugcguugcacuacaacucc cagcaugcccuguugcacug

gacagucagaaguuggucuggcgugagaauag auucucagaucaucucuuggcugaugc

gaggcugauucugagcaaacauungagaucag auugcuggcugccucagcucaccuccc

aaaccaagaguggguugggccuguuagaucac auaggcgacccauacuugguuuca

ggggaugaaucccuuaucuggagacgguugga ugaaucuugcggauaugggacugagc

gggaugaugggacuuguaguccagaaacagcu ucaagccucacuuuuaacgaacuacagguccc accaucccac

ugcuggaguggacaucaaugcaunauuungca aggcguuguaggccucuccagcauu

uagcagcacacagauauuggcaguuucaaccc uaaagcucugccaguucugcuugugcugcuuc a

uagcuuaucagacugauguugacuguuggaug ucauggcaacaacagucgguaggcu

acccuaucaauaungccucugcuuungugcuc ggaguaguagugcaauauugcuuauagggucu

ucaaugaaaucaaccuauccugugguguagga gaaacaggucauagggugauucaucagaca
KB021649:73092695..73092760:-

KB021656:134794298..134794357:+

KB021661:70775875..70775932:-

KB022184:16463..16515:-

KB021653:121432253..121432312:-

KB021653:20533930..20533989:+

KB021657:96570668..96570724:+

KB022003:55963..56021:+

KB021654:118208916..118208990:-

KB021661:70775875..70775932:-

KB028798:1937..2002:+

KB021650:14716187..14716244:+

KB021649:54032702..54032766:+

KB021897:107685..107748:+ 
rsy-miR-novel38-3p

uccuagaagcuugaagccuag

rsy-miR-novel39-5p

uccucaunguacaugcugugugu

rsy-miR-novel40-3p ucuuuuungcuggaacauuucu

rsy-miR-novel41-3p ugcagaugccuguggcaca

rsy-miR-novel44-5p uguaccaugcugguagccagug

rsy-miR-novel46-3p uncacuacuagcagaacucggc

rsy-miR-novel47-5p uugacaucaucauacuugggau ugaggcuuccuacuucaaggcuguguucugac ucauccuagaagcuugaagccuag

uccucaunguacaugcuguguguaucuauuuc ucuuacacagcgcaugcaauguggaua

aaauguuunagaaaaaagacguagucagugc guuuggggaacucuuuuugcuggaacauuuc

agccacugugucuugagauuncuugcagaugc cuguggcaca

uguaccaugcugguagccaguguaaugaaagu ugcuccuggcaaccaguguugugccac

aggaauuccgcuaguucugaacuauuccaugu

uaguaaguaaaaguucacuacuagcagaacuc

ggc

uugacaucaucauacuugggauguaugacaca aagucccaagcaagcugauguuaggc
KB021654:38483781..38483837:-

KB021658:43219725..43219784:+

KB021927:128477..128542:-

KB021662:1930534..1930576:+

KB021656:7549075..7549134:-

KB021658:12977881..12977948:+

KB021760:222321..222379:+ 


\section{That's all Folks!}

